\title{
Design, construction, and evaluation of coal extraction pilot plant to manufacture coal-based carbon pitch
}

\author{
Brian Wayne Bland \\ West Virginia University
}

Follow this and additional works at: https://researchrepository.wvu.edu/etd

\section{Recommended Citation}

Bland, Brian Wayne, "Design, construction, and evaluation of coal extraction pilot plant to manufacture coal-based carbon pitch" (2000). Graduate Theses, Dissertations, and Problem Reports. 1066.

https://researchrepository.wvu.edu/etd/1066

This Thesis is protected by copyright and/or related rights. It has been brought to you by the The Research Repository @ WVU with permission from the rights-holder(s). You are free to use this Thesis in any way that is permitted by the copyright and related rights legislation that applies to your use. For other uses you must obtain permission from the rights-holder(s) directly, unless additional rights are indicated by a Creative Commons license in the record and/ or on the work itself. This Thesis has been accepted for inclusion in WVU Graduate Theses, Dissertations, and Problem Reports collection by an authorized administrator of The Research Repository @ WVU. For more information, please contact researchrepository@mail.wvu.edu. 


\title{
DESIGN, CONSTRUCTION, AND EVALUATION OF COAL EXTRACTION PILOT PLANT \\ TO MANUFACTURE COAL BASED CARBON PITCH
}

\author{
Brian Wayne Bland, BS ChE
}

Thesis Submitted to the

\section{College of Engineering and Mineral Resources}

West Virginia University

In partial fulfillment of the requirements for the Degree of

\author{
Master of Science \\ in \\ Chemical Engineering
}

\begin{abstract}
Alfred Stiller, PhD., Chair
John Zondlo, PhD.

Peter Stansberry, PhD.

Charter Stinespring, PhD.
\end{abstract}

Morgantown, West Virginia

2000 


\section{ABSTRACT \\ Design, Construction, and Evaluation of Coal Extraction Pilot Plant to Manufacture Coal Based Carbon Pitch}

\section{Brian Wayne Bland, BS ChE}

A solvent extraction technique for removing mineral matter, including the inorganic sulfur, from coal has been developed by the Department of Chemical Engineering at WVU. The extraction is preformed at mild conditions $\left(202^{\circ} \mathrm{C}\right.$ and atmospheric pressure) using the solvent N-Methyl-2-Pyrrolidone (NMP). Laboratory results have shown that this technique yields a clean coal product containing less than 1 percent ash and virtually no inorganic sulfur.

This research was conducted for further process design and economic development of an industrial-scale extraction process. Prior to this research, the extraction process was only performed in the laboratory using small-scale equipment. All previous experimental results and economic calculations have been extrapolated from lab data for the scale up to an industrial facility.

This research explored the possibility of production scale processing by constructing a pilot plant. The pilot plant was used to explore the issues of chemical safety, material and energy balance, solvent recovery, and quality of the product. It was necessary to develop an actual flow sheet, prepare a chemical hygiene plan, acquire and assemble equipment, and operate the pilot plant to collect the necessary data.

The Coal Extraction Pilot Plant (CEPU) was designed and built to allow the preparation of significant quantities of coal extract and evaluate the mass and energy balance of the process. The coal used in these experiments showed to be about $36 \pm 2 \%$ soluble in NMP during a 60-minute extraction period. The unit processed $10 \mathrm{~kg}$ of coal per run. Each run through the unit produces about $3.6 \mathrm{~kg}(8 \mathrm{lbs})$ of coal-based carbon extract containing less than $0.6 \%$ ash. There is a solvent retention of less than $0.7 \%$ by weight of processed coal. Assuming all the lost solvent is in the final product, the extract contains less than $2 \%$ NMP by weight.

The Coal Extraction Pilot Unit (CEPU) has been constructed and operated to manufacture significant quantities of clean coal extract. Several ideas brought up and implemented during the construction and operation of the CEPU to enhance its performance. The CEPU is now being utilized to evaluate different possessing methods. 


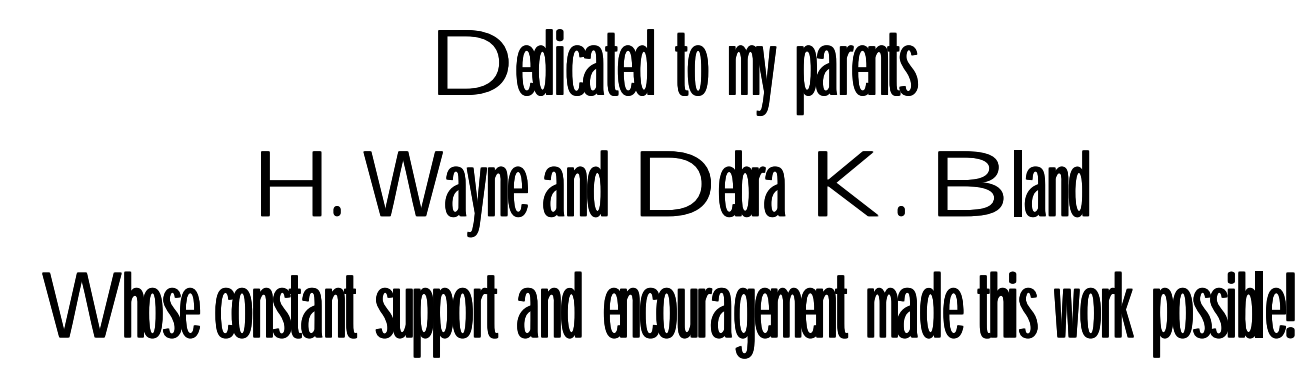




\section{ACKNOWLEDGEMENTS}

The author would like to express his greatest appreciation to Dr. Alfred Stiller for serving as his research advisor. Not only was he a valuable advisor, but also a great friend. His presence and encouragement in the laboratory made this research possible.

My gratitude is also extended to James Hall for all his hours of labor and technical assistance. To Frank Saus for his assistance hard work, support, and enthusiastic efforts. To Dr. Peter Stansberry for dedicating hours of hands-on help and advice for the completion of this work. To Dr. John Zondlo for his discussions, recommendations, encouragement, and support. To Dr. Charter Stinespring I also thank for his advice and encouragement over the past 20 months. To Dr. Dadyburjor for his financial help. Without the help of all these people the research would not have been completed in a timely manner.

I would like to thank those who helped in the lab with experimentation and pure hard labor throughout the course of this project: Byron Walker, Femi Olajide, Chris Taylor, and Raymond Chafin. I would also like to extend a special thanks to Linda Rogers for her secretarial help, and Barbara Bennett for countless hours of proof reading.

Oak Ridge National Laboratory, the Office of Naval Research, the National Research Center for Coal and Energy, and the Department of Chemical Engineering provided the funding for this research project.

I would like to thank certain companies for their valuable support and donations: Koppers Industries, Inc.; Pfaudler, Inc.; F. T. Thomas Co., Inc.; Charles Ross \& Son Co.; Heat Exchange and Transfer, Inc.; and Wilson's Equipment/Northco Corp.

Thanks to my family and closest friends for their affection and encouragement. Above all, I extend my appreciation and gratitude for all those special to me, for their inspiration and unfailing support. 


\section{EXECUTIVE SUMMARY}

A solvent extraction technique for removing mineral matter including the inorganic sulfur from coal has been developed by the Department of Chemical Engineering at West Virginia University. The extraction is preformed at mild conditions $\left(202^{\circ} \mathrm{C}\right.$ and atmospheric pressure) using the solvent N-Methyl-2-Pyrrolidone (NMP). Laboratory results have shown that this technique yields a clean coal product containing less than 1 percent ash and virtually no inorganic sulfur.

Since the development of this extraction technique, research has been conducted in process design and economic development of an industrial-scale extraction process. Prior to this research, the extraction process was only performed in the laboratory using small-scale equipment. Earlier studies have only focused on the laboratory scale approach to this technique. All the experimental results and economic calculations have been extrapolated from lab data for the scale up to an industrial facility. Cost evaluations for a possible industrial scale processes were empirically developed to determine unit cost of this product.

The purpose of this research was to produce a significant amount of coal-based carbon extract. The fundamental complexity of this research is that a large amount of extract-with consistent properties-is required. In the previous research projects preformed by the Chemical Engineering Department at West Virginia University, only small amounts of extract were required. The laboratory facilities could prepare a maximum of 600 grams of extract a week. This required a technician to work full-time in that effort. The previous production scale made it impossible to meet the increased demand in production of extract with the existing laboratory facilities. Therefore, major improvements were required in the extract production facility to satisfy this increase in demand. 
Table of Contents

Chapter

Page

Abstract

ii

Dedication

Acknowledgements iv

Executive Summary $\quad$ V

Table of Contents vi

List of Tables ix

List of Figures $\quad \mathrm{X}$

1 INTRODUCTION 1

1.1 Coal History 1

1.2 WVU NMP Extraction History 3

2 BACKGROUND 5

$2.1 \quad$ What is Coal? 5

$\begin{array}{lll}2.1 .1 & \text { Formation of Coal }\end{array}$

$\begin{array}{lll}\text { 2.1.2 Classification of Coal } & 7\end{array}$

2.1.3 Rank of Coal 9

2.1.4 Petrography: The Components of Coal 10

2.1.5 Organic Component of Coal 11

2.1.6 Inorganic Component of Coal 13

2.1.7 Inorganic Sulfur and Organic Sulfur Contained in Coal 14

2.1.8 Upper Powelton Coal (WVGS 13421) 15

2.2 What is Solvent Extraction of Coal? 15

2.2.1 Solvent Classification 16

2.2.2 Coal and Solvent Properties Affecting Extraction 17

2.2.2 Coal Properties Affecting Extraction 18

2.2.4 Solvent Nature and Physical Properties Affecting Extraction 18

2.2.5 Parametric Study of Solvent Extraction 19

2.2.5.1 Effect of time and temperature (kinetics of solvent extraction) 20

2.2.5.2 Effect of particle size on extraction Yield 21

2.2.5.3 Effect of oxygen and moisture on extraction 21 
$2.3 \quad$ N-methyl-2-pyrrolidone (NMP), The Solvent of Choice? 24

2.4 What is the Product of Solvent Extraction of Coal? 27

Pilot Plant Construction Timeline

Preparing the Necessary Extract to Fulfill the ORNL Contract 42 
4.3.1

4.3.2

4.3.3

4.3.4

4.4

4.5

4.3.2

4.3 .3

5

5.1 .0

5.2 .0

5.2 .1

5.2 .2

5.2 .3

5.2 .4

5.2 .5

6

6.0 .1

6.0 .2

6.1 .0

6.1 .1

6.1 .2

6.2 .0

6.3 .0

6.4 .0

7

8
TFE Supporting Heat Exchanger

(HE-102) 59

Vacuum Pump for TFE System

Vapor Trap Heat Exchanger

Product or "Thickened Extract" Tank

Condensed Solvent (NMP) Collection

Rotovap

Drying Ovens

Vacuum Pump for Rotovap \& Drying Ovens

Vapor Trap/Condenser Heat Exchanger

MATERIAL BALANCE

Overall Material Balance

Material Balance Over Individual Units

Material Balance Over Reactor (CSTR)

Material Balance Over Centrifuge

Material Balance Over TFE

Material Balance Over Rotary Evaporator

Material Balance Over Drying Ovens

ENERGY BALANCE

Thermal Properties of NMP

Thermal Properties of Coal

Energy Balance on Reactor (CSTR)

Reactor $\mathrm{NMP} / \mathrm{H}_{2} \mathrm{O}$ Vapor Condenser

Reactor Slurry Cooler Before Centrifuge

Energy Balance on Wiped Thin Film Evaporator

Energy Balance on Rotary Evaporator (Rotovap)

Energy Balance on Drying Ovens

RESULTS

RECOMENDATIONS

REFERENCES
(VP-101) 60

(HE-103) 60

(T-102) 60

(T-103) 60

(RV-101) 61

(DO-101-3) 63

(VP-102) 64

(HE-105) 64

65

66

68

(R-101) 68

(Ct-101) 69

(TFE-101) 69

(RV-101) 69

(DO-101-3) 70

72

72

72

(R-101) 74

(HE-101) 77

(HE-104) 77

(TFE-101) 79

(RV-101) 82

(DO-101-3) 83

84

86

91 
$\underline{\text { List of Tables }}$

Item

Title

Page

Table 2-1

Classification Profile Chart

8

Table 2-2

ASTM Standard Classification of Coal by Rank

10

Table 2-3

Key Physical and Chemical Properties of

26

N-Methyl-2-Pyrrolidone

Table 2-4

Potential Uses of Coal Extracts

28

Table 3-2

Coal Extraction Pilot Unit Stream Table

45

Table 3-3

Equipment List for Coal Extraction Pilot Unit

46

Table 5-1

Material Balance Results

70

Table 6-1

TFE Normal Operating Parameters

81 


\section{List of Figures}

Item

Figure 2-1

Figure 2-2

Figure 2-3

Figure 2-4

Figure 3-1

Figure 3-2

Figure 3-3

Figure 3-4

Figure 3-5

Figure 3-6

Figure 4-1

Figure 4-2

Figure 4-3

Figures $4-4 \& 5$

Figures 4-6\&7

Figures 4-8\&9

Figure 4-10
Title

Raw Coal

Page

5

Schematic Representation of Coalification Process. (Speight, 1983)

High-Volatile Bituminous Coal Model

12

Showing the Functional Groups

Heteroatom Functionalities in Coal

Block Flow Diagram for Laboratory Production of Coal-Based Carbon Pitch

Coal-Based Carbon Pitch

CEPU Construction Time Task Deliverable Chart

P-101 Pump Curve

Process Flow Diagram for

Production of Coal-Based Carbon Pitch

Production of Coal-Based Carbon Pitch

Continuous Stirred Tank Reactor with Heat Exchanger,

48

Pump, Condenser, and Absorber

CSTR Lid Showing Motor, Cap, and Condenser

Sharples Penwalt BM-PF 290 Centrifuge

52

Pfaudler $1.2 \mathrm{ft}^{2}$ Wiped Thin Film Evaporator

56

Thickened Extract Leaving Wiped Thin Film Evaporator 58

Modified Buchi R-135 Rotary Evaporator with Steel Tank 59

Processed Coal and Pitch Drying Ovens

62 and Heat Exchanger 
Item

Title

Figure 4-11

Drying Oven Condenser (HE-105)

Page

$\&$ Collection Tank (T-106)

Figure 5-1

Block Flow Diagram with Stream Compositions for

83

Production of Coal-Based Carbon Pitch

Figure 6-1

Typical Values of the Specific Heats of Coal,

72

Coke, and Graphite. (Volborth 1987)

Figure 6-2

CSTR (R-101) Temperature Profile for Heating

Figure 6-3

CSTR (R-101) Temperature Profile for Cooling the

75

Reactor Without Supporting Heat Exchanger HE-104

Figure 6-4

CSTR (R-101) Slurry Cooling Before Centrifuge (Ct-101) 77

With Supporting Heat Exchanger (HE-104)

Figure 6-5

Log of NMP Saturation Pressure vs. Temperature

Figure 8-1

Charles Ross \& Son Co. (HDM-40)

89

40-gal Hydraulic Double Planetary Mixer

Figure 8-2

Hydraulic Double Planetary Mixer

89

with Supporting Condenser

Figure $8-3 \& 4$

Double Planetary Mixing Blades with Wall Scraper

90

Figure $8-5 \& 6$

Double Planetary Mixing Blades

90

With Product Before and After Grinding 


\section{CHAPTER 1 \\ INTRODUCTION}

\subsubsection{Coal History}

Coal has been considered a valuable raw material throughout history. The first known use of coal was as a domestic heating source in China around 1000 B.C (Speight 1983). During the Middle Ages coal was used extensively in Europe as a domestic heating source and a substitute for charcoal by blacksmiths. The Industrial Revolution promoted the widespread use of coal during the mid- $19^{\text {th }}$ century. Coal ushered in the Industrial Revolution by serving as a cheap and abundant carbon source needed to produce coke for large-scale iron production (Schobert 1987, Zondlo 1995).

The uses of coal are not limited to just iron production and heating. Prior to World War II, coal was used extensively in the United States and Germany to make chemicals such as ammonia, methanol, and benzene. During World War II, Germany's imported supplies of natural rubber and petroleum were cut off. They turned to coal to fuel their war effort. The German tanks and planes ran on synthetic gasoline and rubber made directly from coal gasification and liquefaction. After the war, the use of coal in the United States and Germany declined in the late 1950's because cheap and abundant supplies of natural gas and petroleum became available. Petroleum and natural gas have since been the dominant raw materials for gasoline, rubber, and the manufacturing of specialty chemicals. However, as early as 1970, there has been renewed interest in coal.

As domestic petroleum supplies become less available, the United States has become more dependent upon foreign oil. As a result, the United States has historically been at the mercy of the Middle East oil prices. In the early 1970's, the Organization of Petroleum Exporting Countries (OPEC) imposed an oil embargo on the United States that drastically increased the price of gasoline and other petroleum products. At the present, political unrest in the Middle East has intensified again, and the price of imported oil from the Middle East reflected the escalation. Political wars such as the Persian Gulf War and Desert Storm, and the OPEC monopoly, have pushed oil prices to over $\$ 36.00$ per barrel. The cost per barrel has more than doubled over the past year. The United States was economically unprepared for this increase in oil price, but was forced to pay 
these high prices because it is unable to extricate itself from its oil dependency on the Middle East. Even though the oil prices are elevated, the cost is not enough to make coal gasification and liquefaction economical. In spite of current oversupply of natural gas and oil, and the fluctuations resulting from international competition and shortsighted planning, there is no doubt that coal will constitute in the near future the major organic energy source for hundreds of years to come. Coal combustion for electric energy and the use of coal as a raw material for the organic chemistry industry are and will remain the basis for modern prosperity (Volborth 1987),

The advancement of technology has allowed the extraction of more petroleum and natural gas with greater efficiency. Technology today allows reserves to be reached that were never envisioned possible. Unlike coal, sulfur content has never been a problem for the petroleum industry until recently. The deeper the reserves, the more sulfur content in the crude produced. As petroleum production rate goes up to meet consumption needs, so does the sulfur content in the crude oil and natural gas.

The volatility of the foreign petroleum market and increasingly stringent environmental regulations make it important that economically and environmentally sound methods be developed which will allow for the utilization of U.S. coal reserves. Utilizing these domestic coal reserves will not only reduce our dependence on foreign oil, but will boost the economy of the United States.

The elevated cost of petroleum combined with its increased sulfur content has dramatically increased the cost of manufacturing specialty chemicals from petroleum. With the energy crisis and the belief that natural gas and petroleum supplies are extremely limited, a large amount of time and money has been spent on coal gasification, liquefaction, and the manufacturing of specialty chemicals and products from coal.

Billions of dollars have been spent over the last 50 years on research, design, evaluation, and construction of numerous processes to convert coal into similar feedstocks that are currently produced by the petroleum industry (Bonifera 1997).

Many of the processes were found to be technically feasible, but not economical. Most are too costly when compared to the current prices of the feedstocks that are currently produced from oil and natural gas. Coal comprises 75 percent of the energy reserves in the United States. It is estimated that there is enough reserves for the coal 
industry to last for another 100 to 300 years. With the current rate of production of petroleum and natural gas, it as thought that coal would long outlast the petroleum industry (Schobert 1987, Zondlo 1995). However, a significant amount of the coal reserves in the United States contains high levels of sulfur and mineral matter hindering its use in present industrial process.

\subsubsection{WVU NMP Extraction History}

For the past several years, the Department of Chemical Engineering at West Virginia University (WVU) College of Engineering and Mineral Resources (CEMR) has been conducting research into the use of coal as a raw material for producing feedstocks for carbon products. The focus of these studies has been the dissolution of upper Powelton and other bituminous coals in an aprotic dipolar solvent. This research has led to the development of a solvent extraction technique that uses $\mathrm{N}$-methyl-2-pyrrolidone (NMP) to separate the mineral matter (referred to as ash) and inorganic sulfur from the carbon components of coal. This solvent has been shown to dissolve over $50 \%$ of the total carbonaceous carbon material in coal. In sequential steps the insoluble materials including the ash and some organic material are separated from the soluble materials by using a centrifuge or pressure filter. The filtrate, that contains all the soluble coal materials, is evaporated to dryness and a dry "solvent free" solid, carbonaceous material that contains less than 1 percent ash and no detectable pyritic or inorganic sulfur results (Gerstner [1989]). This solid, referred to as "reconstituted coal", coal based carbon pitch, extract, or just pitch has unique properties.

1) The BTU has increased to over $14,500 \mathrm{BTU} / \mathrm{lb}$, as compared to the original Powelton coal, which averages at 12,500 BTU/lb. This increase in BTU value is because the ash, which has little BTU value, has been removed.

2) The pitch produces an ash-free isotropic coke. When graphitized the isotopic coke produces graphite that has very small crystallite domains. Graphite, by its very nature is anisotropic. However, when graphite is produced from coke made from the reconstituted coal, it appears as a mosaic of very small randomly oriented anisotropic graphite crystallites. The final graphite has bulk properties that are isotropic in character. Hence it is referred to as isotropic graphite. 
3) The carbon yield (amount of fixed carbon after coking) of the reconstituted coal is about $63 \%$, which is fairly high. Ideally, a higher carbon yield, such as $73 \%$, would be preferable for the carbon material industry.

This newly-developed coal extraction technique of de-ashing and extraction of coal using N-methyl-2-pyrrolidone (NMP) at atmospheric pressure and moderate temperature $\left(202^{\circ} \mathrm{C}\right)$ has been carefully studied at WVU by the Chemical Engineering Department. These studies, such as those by K. Renganathan (1986), G. R. Phillips (1988), J. A. Gerstner (1989), S. D. Gadam (1990), V. P. Bonifera Jr. (1997), W.R. Stewart (1992), etc, only focused on the laboratory scale approach to this new technique. Cost evaluations of possible industrial scale of any kind were empirically developed to estimate cost per ton of this product.

The research presented in this thesis explores the scale-up of production by constructing a pilot plant that will permit more complete material and energy balances. Until now, no pilot plant existed to address the issue of actual production of any appreciable quantity of the coal-based carbon extract. Limited data existed to upscale the process toward industrial scale production. For the most part the issues of chemical safety, quantity, solvent recovery, quality of the product, and material handling and processing had not been satisfactorily addressed. The majority of the data collection on the extraction technique of coal based carbon product was preformed in a laboratory using closely controlled conditions. The primary goal of this research is to develop an actual flow sheet, and complete design and construction for a pilot plant at WVU.

This thesis reports the design, construction, operation, and preliminary evaluations of a pilot scale operation for a coal based Carbon Extraction Pilot Unit (CEPU). The specific objectives for this research include:

1. design and construction of the CEPU;

2. material Balance;

3. energy balance;

4. and process improvements for production. 


\section{CHAPTER 2}

\section{BACKGROUND}

It is difficult to write any extensive formal treatise in the field of coal research without providing some background on the very basics of coal science. Therefore, this chapter is dedicated to explaining a few general areas that greatly impact the science of solvent extraction of coal. This chapter gives an introduction to the nature of coal, what is solvent extraction of coal, and what is the product of solvent extraction. It explains why upper Powelton (WVGS 13421) coal was chosen for this research, why N-methyl-2pyrrolidone was selected as the solvent, and how the product of that solvent could be used.

\subsubsection{What is Coal?}

Coal is actually a generic term given to widespread and greatly differing natural rocks that consist primarily or organic material mixed with smaller quantities of inorganic materials. Coal can be defined as a compact stratified mass of lithified plant material. This plant material has been modified chemically to varying degrees, and interspersed with smaller amounts of inorganic

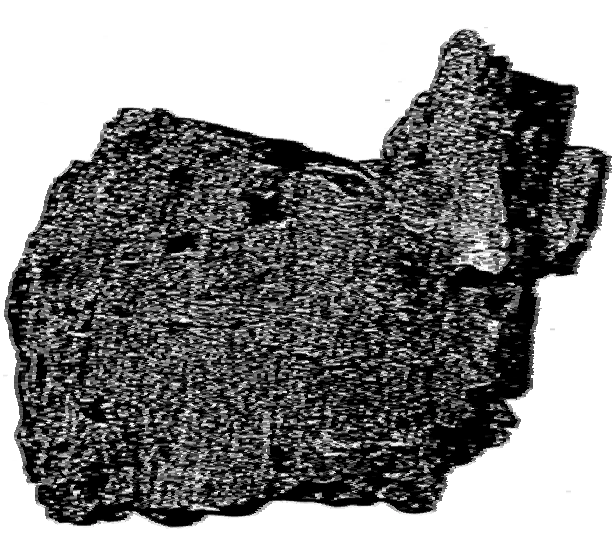

Figure 2-1 Raw Coal materials (Francis 1954). Essentially, plant material builds up and partially decomposes without oxygen to form a mixture of organic and significant inorganic mineral matter, which is then compressed to form a heterogeneous carbonaceous rock of organic origin.

This rock varies from region to region and era to era. Coal can be descried as a fruitcake that is formed from a mixture of diverse ingredients then baked to produce a visibly heterogeneous product as shown in Figure 2-1. The ingredients are composed of trees, ferns, rushes, lyco-pods, and several thousand-plant species that have been identified in coal beds. Plant remains of similar types have been found in all types (ranks) of coal, but the relative amounts vary considerably. Therefore, the composition of coal varies considerably. 


\subsubsection{Formation of Coal}

Coal is formed from the collection of plant debris under the correct conditions. This formation usually takes place in a swamp or shallow lake where the debris accumulates and partially decomposes to a material called peat. The peat then becomes lithified in what is termed the "coalification" process. The coalification process shown in Figure 2-2 is a progressive change in the plant debris as it is transformed from peat to lignite and then through the higher ranks of coals to anthracite. The degree of coalification generally determines the rank of the coal. This process is not a series of straightforward chemical changes; rather, it is a metamorphosis. The metamorphosis of the plant debris not only relies on time but also temperature and pressure. The major influences in the coalification process are believed to be the resulting heat and pressure developed because of the overlying sedimentary cover (overburden). Under the correct conditions, oxygen and hydrogen are progressively eliminated. The ultimate final product contains about $90 \%$ by weight carbon, i.e., anthracite (Speight 1983).

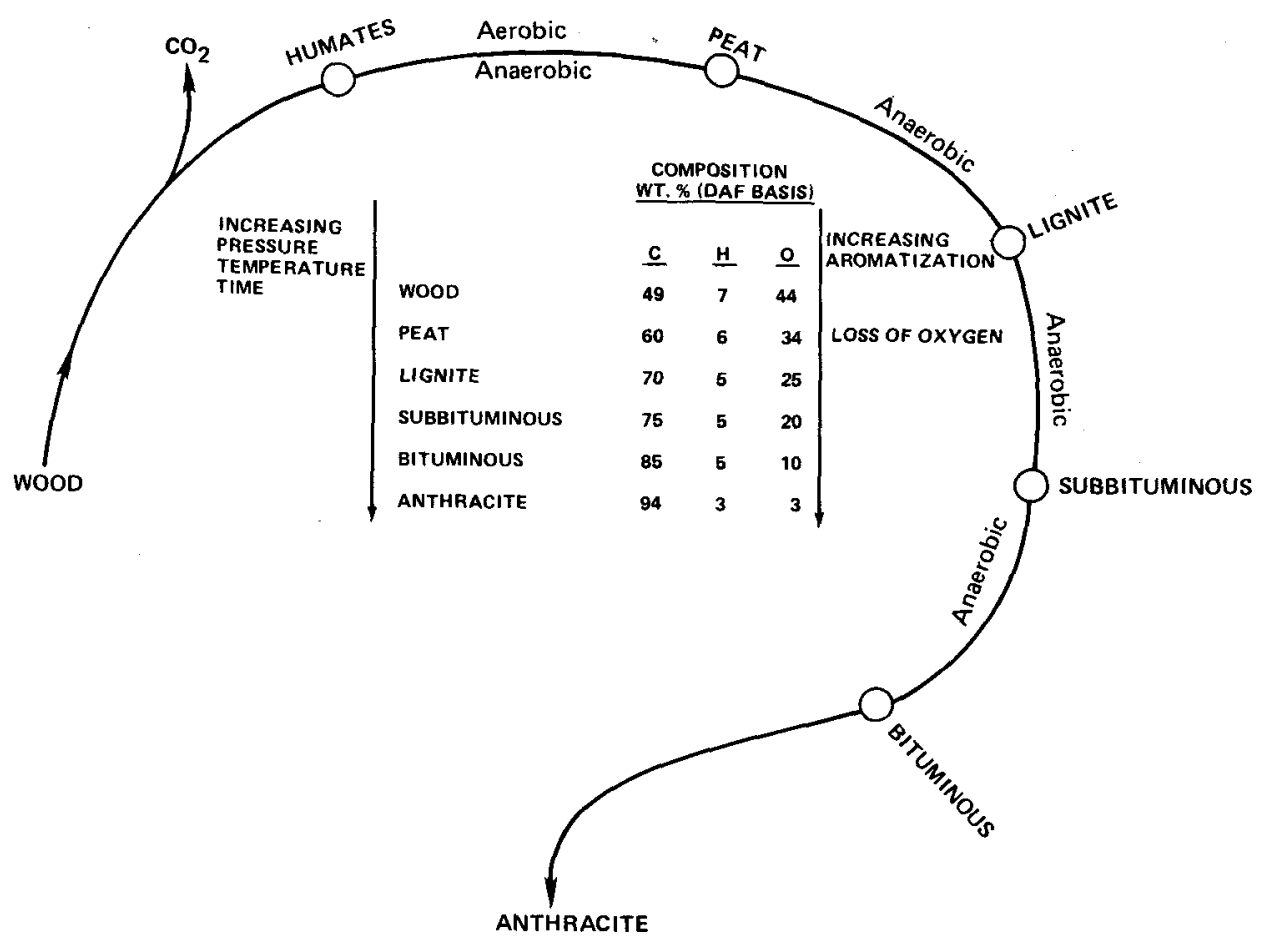

Figure 2-2 Schematic Representation of Coalification Process. (Speight, 1983) 


\subsubsection{Classification of Coal}

As described earlier, coal is actually a generic term given to widespread and greatly differing natural rocks that consist primarily or organic material mixed with smaller quantities of inorganic materials. Coal ranges from a soft, moist, brownish material to a very hard, glossy, black solid. With the wide span of compositions and properties, there is a need to have some standard way of describing and classifying coal. The traditional procedure of characterizing coal relies on the following three types of analysis (Schobert 1987).

A) Proximate Analysis: The first method of examining coal is used to evaluate its fuel potential. This method of coal analysis was developed as a simple means for classifying coal by determining the product distribution from coal during heating under standard conditions. Proximate analysis of coal is expressed in terms of the following four constituents:

1) Moisture-Moisture or water is lost upon heating coal to $105^{\circ} \mathrm{C}$

2) Volatile matter-The gases and vapors driven off during pyrolysis. (i.e. heating to $800^{\circ} \mathrm{C}$ in the absence of oxygen).

3) Fixed Carbon-The carbon remaining as the nonvolatile fraction of the pyrolysed coal.

4) Ash-The mineral matter or "ash" that is left after complete combustion of coal, or by heating to $750^{\circ} \mathrm{C}$, in the presence of oxygen.

Results from proximate analysis confirm the observation of great variability among coals. The moisture content in coals ranges from nearly zero to almost $70 \%$, the volatile matter ranges from 5 to $60 \%$, while the ash content varies from 1 to $35 \%$, and fixed carbon ranges from 20 to $85 \%$. Proximate analysis is relatively quick and easy to perform and is useful in making experimental predictions of the behavior of coal and classification type.

B) Heating Value: This analysis determines the amount of energy the can be obtained by burning the coal, and is termed as the heating value or calorific value of coal. This value is usually reported in BTU/lb. 
C) Ultimate Analyses: This analysis is done using IR spectroscopy or the following set of analytical procedures to determine the elemental composition of coal in terms of the weight percentages of carbon, hydrogen, nitrogen, oxygen, and sulfur.

Reacting the coal and separating the materials produced determine the compositions of these elements. (1) Carbon and hydrogen: Burning the coal and collecting the resulting carbon dioxide and water in chemicals that can absorb them is used to measure the carbon and hydrogen. These chemicals are weighed to determine the weight gain and calculate the amount of carbon and hydrogen consumed. (2) Sulfur: One of the common methods to determine the sulfur is to convert it to sodium sulfate, which can then be converted to barium sulfate. The Barium sulfate is collected dried and weighted. (3) Nitrogen: Nitrogen is converted to ammonia, which is then absorbed in a known amount of acid. The difference in the acid is used calculate the amount of ammonia made during the experiment. (4) Oxygen: There is no simple analytical method for determining the amount of oxygen. The most commonly used method today involves bombarding the coal with neutrons to convert the oxygen to unstable isotope of nitrogen (nitrogen-16). The nitrogen-16 rapidly decays emitting beta $(\beta)$ and gamma $(\gamma)$ rays, which can be detected and counted. Table 2-1 shows the average ultimate analysis of different classifications of coals.

\section{Table 2-1 Classification Profile Chart}

\begin{tabular}{|c|c|c|c|c|c|c|c|c|c|}
\hline \multirow[b]{2}{*}{ Class } & \multirow{2}{*}{\multicolumn{2}{|c|}{ Group }} & \multicolumn{7}{|c|}{ Average Analysis-moisture and ash-free } \\
\hline & & & $\begin{array}{c}\text { Volatile } \\
\text { Matter } \\
(\%) \\
\end{array}$ & $\begin{array}{c}\text { Hydrogen } \\
\text { (wt.\%) }\end{array}$ & $\begin{array}{c}\text { Carbon } \\
(\text { wt.\%) }\end{array}$ & $\begin{array}{c}\text { Oxygen } \\
\text { (wt.\%) }\end{array}$ & $\begin{array}{c}\text { Heating } \\
\text { Value } \\
(\mathrm{kJ} / \mathrm{kg})^{\mathrm{a}}\end{array}$ & $\mathbf{C} / \mathbf{H}$ & $(\mathbf{C}+\mathbf{H}) / \mathbf{O}$ \\
\hline \multirow[t]{3}{*}{ I. Anthracite } & 1 & Meta-anthracite & 1.8 & 2.0 & 94.4 & 2.0 & 34425 & 46.0 & 50.8 \\
\hline & 2 & Anthracite & 5.2 & 2.9 & 91.0 & 2.3 & 35000 & 33.6 & 42.4 \\
\hline & 3 & Semianthracite & 9.9 & 3.9 & 91.0 & 2.8 & 35725 & 23.4 & 31.3 \\
\hline \multirow[t]{5}{*}{ II. Bituminous } & 1 & Low volatile bituminous coal & 19.1 & 4.7 & 89.9 & 2.6 & 36260 & 19.2 & 37.5 \\
\hline & 2 & Medium-volatile bituminous coal & 26.9 & 5.2 & 88.4 & 4.2 & 35925 & 16.9 & 25.1 \\
\hline & 3 & High-volatile A bituminous coal & 38.8 & 5.5 & 83.0 & 7.3 & 34655 & 15.0 & 13.8 \\
\hline & 4 & High-volatile B bituminous coal & 43.6 & 5.6 & 80.7 & 10.8 & 33330 & 14.4 & 8.1 \\
\hline & 5 & High-volatile $\mathrm{C}$ bituminous coal & 44.6 & 4.4 & 77.7 & 13.5 & 31910 & 14.2 & 6.2 \\
\hline \multirow{3}{*}{\multicolumn{2}{|c|}{ III. Subbituminous }} & Subbituminous A coal & 44.7 & 5.3 & 76.0 & 16.4 & 30680 & 14.3 & 5.0 \\
\hline & & Subbituminous $\mathrm{C}$ coal & 42.7 & 5.2 & 76.1 & 16.6 & 30400 & 14.7 & 5.0 \\
\hline & 3 & Subbituminous B coal & 44.2 & 5.1 & 73.9 & 19.2 & 29050 & 14.6 & 4.2 \\
\hline \multirow[t]{2}{*}{ IV. Lignite } & 1 & Lignite A & 46.7 & 4.9 & 71.2 & 21.9 & 28305 & 14.5 & 3.6 \\
\hline & 2 & Lignite B & - & - & - & - & - & - & - \\
\hline
\end{tabular}

${ }^{\mathrm{a}}$ To Convert $\mathrm{kJ} / \mathrm{kg}$ to Btu/lb, divide by 2.326

Source: Hensel (1981) 


\subsubsection{Rank of Coal}

Coal may seem to be a homogeneous substance, but it is not. Like the vegetation from which it was formed, coals composition varies greatly from one region to another. The vast variety and complexity of the properties and composition of coal made it necessary to develop a procedure to classify it. The classification of some of these properties was necessary for the selection of a standard way to rank or classify different coals for research. Many coal properties and numerous items of commercial description have been proposed as a basis for categorizing coal. The most common of these is "rank", which is a measure of the degree of coalification that the organic plant sediment has reached in its metamorphosis from peat to near graphite-like material. Changes in the metamorphosing coal can be generalized as follows (Smith, et al. 1994): (a) moisture content decreases, rapidly in the early stages, slowly in the later stages; (b) carbon content increases while oxygen content decreases; (c) hydrogen content changes little until late stages of coalification where it decreases rapidly; (d) volatile matter generally decreases; (e) calorific value increases, levels off, then drops off slightly.

A set of labels for different kinds of coal could help separate coals into classifications having roughly similar properties. For coal in the United States, the classification is made on the basis of rank, and the names of the coal ranks are labels that provide some information about the coal. The rank of coal indicates the extent of the geological maturity of the coal, the carbon content, the volatile matter, and the heating value. In the United States the American Society for Testing and Materials (ASTM) developed the system that is currently used for classification of coal by ranks.

Classification of coal by rank is specified from knowledge of the proximate analysis and heating analysis (calorific value) of a coal. Rank is not necessarily directly related to the carbon content of coal or any other specific coal structure. There are four main ranks of coal. These ranks are further broken done into groups to classify the coal. The ASTM Designation D 388-84 (1984), as reproduced in Table 2-2, is the standard specification for the classification of coal by rank in North America. In this system the higher rank coals are specified by the percentage of fixed carbon from the proximate analysis on a dry, ash-free basis. Lower rank coals contain less than $69 \%$ fixed carbon, 
and are classified by their calorific value on a moist basis. Agglomerating characteristics of coals are used to distinguish between groups, such as between subbituminous $\mathrm{A}$ and high volatile C bituminous (ASTM 1984).

\section{$\underline{\text { Table 2-2 }} \quad$ ASTM Standard Classification of Coal by Rank ${ }^{\text {a }}$}

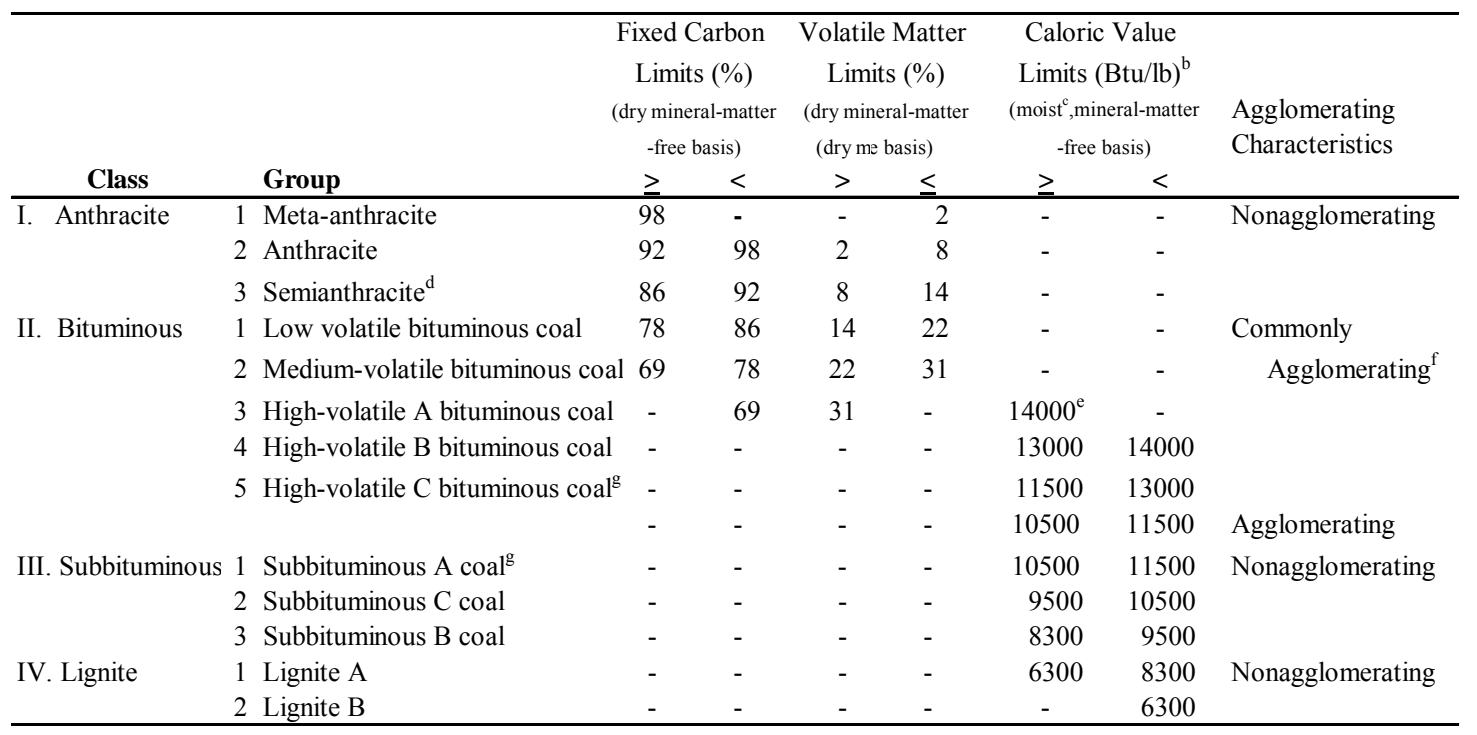

${ }^{\text {a }}$ This classification applies to coals composed mainly of vitrinite; coals rich in liptinite or inertinite do not fit into this classification system.

${ }^{\mathrm{b}}$ Standard units for ASTM classification for calorific value are Btu/lb. To convert to SI units of $\mathrm{kJ} / \mathrm{kg}$, multiply Btullb by 2.326 .

${ }^{\mathrm{c}}$ Moist refers to coal containing its natural inherent moisture but not including visible water on the surface of the coal. .

${ }^{\mathrm{d}}$ If agglomerating, classify in low-volatile group of the bituminous class.

${ }^{\mathrm{e}}$ Coals having $69 \%$ or more fixed carbon on a dry, mineral-matter-free basis are classified according to fixed carbon only, regardless of calorific value.

${ }^{\mathrm{f}}$ It is recognized that there may be nonagglomerating varieties in these groups of bituminous class, and that there are notable exceptions in the high-volatile $\mathrm{C}$ bituminous group.

g Agglomerating coals in the range 10,500-11,500 Btu/lb are classed as high-volatile C bituminous coal.

Source: ASTM Designation D 388-84 (1984).

\subsubsection{Petrography: The Components of Coal}

Coal petrography is a visual examination of coal revealing basic chemical and physical properties of its constituents. These visual observations can be preformed with the naked eye or with a microscopic. Microscopic visual observations reveal what are called macerals. This is used in parallel to rock layers built of minerals. Macerals (mas'er-als) are organic substances derived from plant tissue, cell contents, and animal remains that were incorporated into sedimentary strata, and then altered physically and chemically by natural processes (Gorbaty et al., 1981). 
The macerals in coal tend to be aromatic in nature. The carbon content of coal can be classified as aromatic, hydroaromatic, or aliphatic. The aromaticity of coal increases with rank with $40-50 \%$ for subbituminous coal, and over $90 \%$ for anthracite (Larsen 1978). In a typical bituminous coal, 65 to $75 \%$ of the total carbon is aromatic, 15 to $25 \%$ is hydroaromatic and 5 to $10 \%$ is aliphatic.

Bands of material are distinguished running through a sample of bituminous coal with the naked eye. These bands are called lithotypes. Coal lithotypes arise from different conditions that existed during the coalification process of the coal. Coal contains the following four types of these bands (Schobert 1987):

1) Vitrain - a glossy black material that breaks in a circular pattern (called conchoidal fracture) similar to the way glass breaks when chipped;

2) Clarain - a glossy black material that breaks horizontally;

3) Durain - a dull black material having a granular appearance; and

4) Fusain - a material that resembles charcoal.

Lithotypes are composed of macerals, which can be distinguished under a microscope. The three main macerals groups are vitrinite, exinite, and inertinite. Vitrinite is derived from variously decomposed woody tissues. Exinite (liptinite) is derived from spores and pollen coats, cuticles, resins, and other fatty secretions. Inertinites are derived mainly from partial carbonization of various plant tissues in the peat stage.

\section{$\underline{\text { 2.1.5 }}$ Organic Component of Coal}

The organic matrix of the coal consists mainly of carbon with a smaller amount of hydrogen, oxygen, nitrogen, and sulfur. Coal is actually an aggregate of microscopically distinguishable, physical distinctive, and chemically different macerals and minerals (Gorbaty 1982).

The organic materials in coal are derived mainly from plant remains that have undergone various degrees of decompositions in the peat swamps and various degrees of chemical and biochemical alteration after burial (Meyers 1982). These organic components are composed of large polymer like carbonaceous molecules as exemplified in Figure 2-3 (Wiser 1975). The physical and chemical properties of these molecular structures determine the coal properties (Gorbaty et al., 1982). 


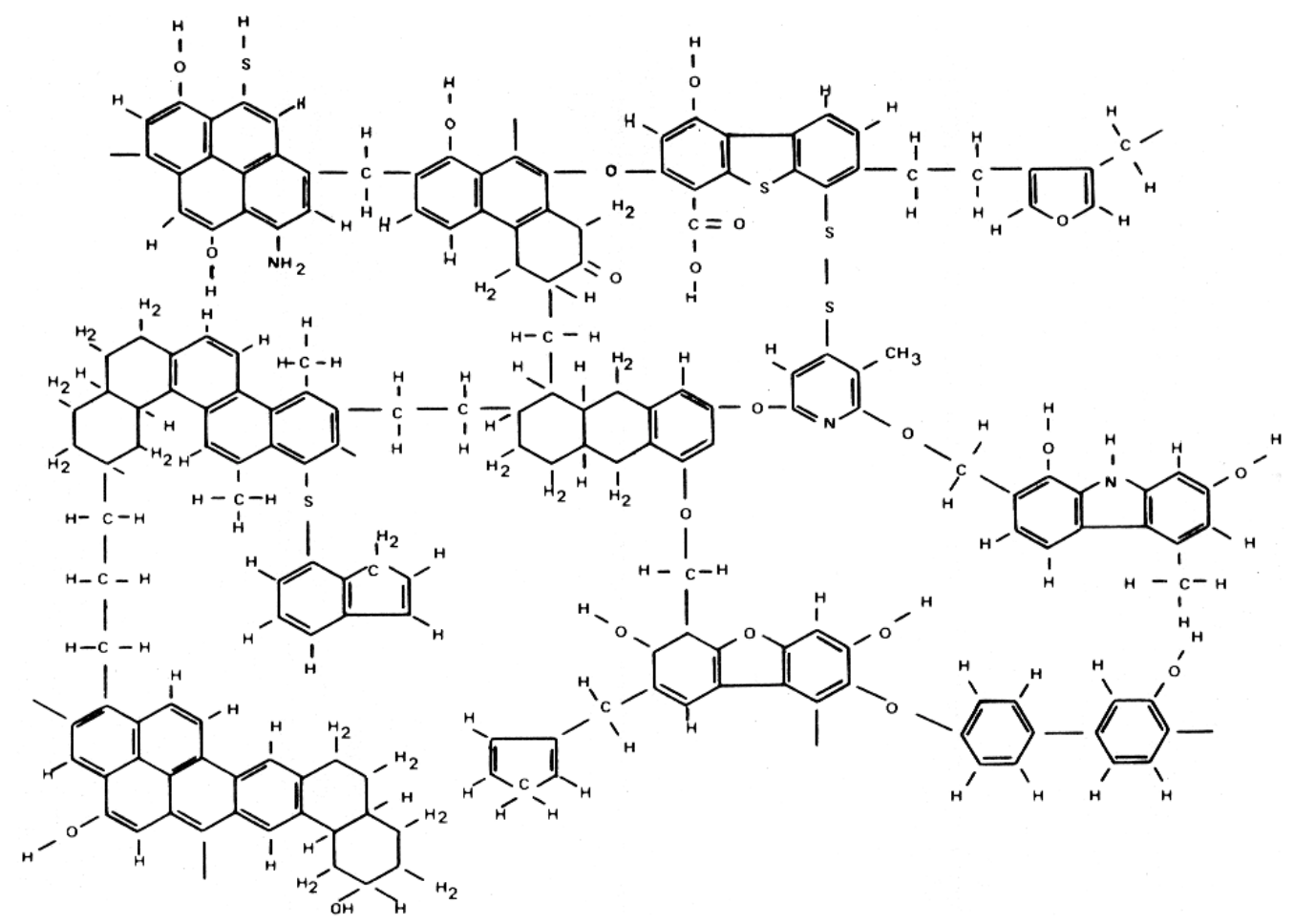

\section{Figure 2-3 High-Volatile Bituminous Coal Model Showing the Functional Groups}

The major chemical functionalities identified in the coal include hydroxyls, carbonyls, aliphatic bridges, heterocylic ring units, and aromatic ring units (Larsen 1978). A typical bituminous coal consists of clusters of aromatic and hydroaromatic carbon rings. Figure 2-4 shows the major heteroatom functional group types found in coal. Oxygen occurs predominately as phenolic or etheric groups with fewer amounts of carboxylic acids or esters. Sulfur has about the same heteroatom functional groups as oxygen in coal although sulfoxides are not as well defined. Nitrogen occurs predominately as pyridine or pyrrolic type rings (Larsen 1978). 
Oxygen<smiles>Oc1ccccc1</smiles>
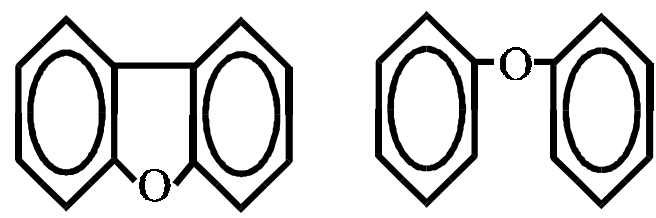

Sulfur<smiles>Sc1ccccc1</smiles><smiles>c1ccc2c(c1)CCS2</smiles><smiles>c1ccc(Sc2ccccc2)cc1</smiles>

Nitrogen
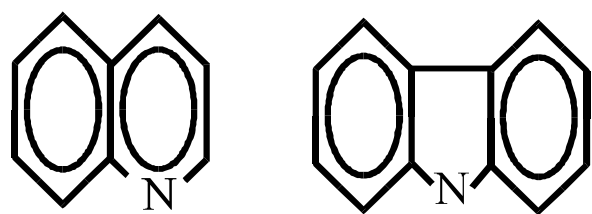

\section{Figure 2-4 Heteroatom Functionalities in Coal}

\subsubsection{Inorganic Component of Coal}

The inorganic matter normally found in coal consists mainly of the minerals deposited with the coal when it was formed. The inorganic minerals are different from ash. The ash is the inorganic oxides that are left after complete combustion of the coal. This inorganic or mineral matter is derived from several sources: transported and deposited by water or air, direct precipitation from solution, precipitation by organic metabolism, etc. The mineral matter deposited in the coal is mainly in the form of clay (aluminosilicates), carbonates, sulfide ores, oxide ores, quartz (silica), and phosphates (Speight 1983). The mineral content of coal can be as high as 35\%. The minerals are usually present in the coal as layers or bands (Meyers 1982). These bands or layers are caused by sedimentation of inorganic substance between layers of organic matter. When the coal is burned these minerals are oxidized to form ferric oxide, calcium oxide, 
magnesium oxide, sulfur oxide, etc. These oxides are the main constituents of the coal ash.

\subsubsection{Inorganic Sulfur and Organic Sulfur Contained in Coal}

Of the eighty elements found in coal, the one beside carbon having the most significant effect on coal use is sulfur. When the sulfur in coal is burned, it produces sulfur oxides that are felt to produce acid rain. The chemistry of the production of the sulfur oxides has been extensively studied. The sulfur in coal has prompted the spending of billions of dollars on equipment and processes to capture the sulfur oxides or to reduce the sulfur content of the coal before it is burned. All coals contain some degree of sulfur. The amount ranges from $0.1 \%$ to $10 \%$ by weight (Eliot 1978). The average in most coal samples ranges from 1 to $4 \%$ by weight. Traditionally the sulfur in coal can be classified as either inorganic or organic. The inorganic sulfur is further broken down into pyritic sulfur and sulfate sulfur as follows:

1) Organic sulfur - is a part of the molecular composition of the coal itself.

2) Pyritic sulfur - occurs in the mineral pyrite and some related minerals.

3) Sulfate sulfur-is mostly iron sulfates.

Organic sulfur, which cannot be removed by physical separation, comprises from 30 to $70 \%$ of the total sulfur contained in coal. Generally, the organic sulfur/total sulfur ratio is the highest for low sulfur coals and decreases as the total sulfur content increases (Eliot 1978). In most bituminous coals, the majority of the sulfur is pyritic sulfur (Schobert 1987).

Pyritic sulfur is sulfur bonded with iron. It is commonly the mineral pyrite or fools gold, and occurs occasionally in coal as discrete crystals but also as microscopic particles. It is classified as a dense mineral with a specific gravity of about 5.0, where as coal has a maximum specific gravity of 1.8. Pyrite content of a coal can be reduced significantly by size reduction and subsequent specific gravity separation.

In most coals, sulfate sulfur is only a small fraction of the total sulfur (less than $0.05 \%$ ). The sulfate is normally an oxidation product, which is water-soluble and can easily be removed. The problem is that this sulfur, although it is low in concentration, it is also soluble in most solvents. 


\subsubsection{Upper Powelton Coal (West Virginia Geological Surveys \# 13421)}

Upper Powelton (WVGS 13421) coal was chosen for this research based on its availability and solubility in NMP. Leckie Smokless Coal Co. donated all the coal for this research to West Virginia University. The average dissolution for this coal in NMP has yields as high as $38 \%$. In our current extraction unit, Upper Powleton coal typically yields about $36 \pm 2 \%$.

\subsubsection{What is Solvent Extraction of Coal?}

Solvent extraction is the method of using a solvent to dissolve a desired portion of coal and extract or separate it from the undesired portion of coal. This can be explained by comparing it to the separation of sugar and rice with water. Imagine that the coal is actually made of a solid mixture of sugar and rice. If this mixture is placed into water, the sugar will dissolve, but the rice will remain unaffected. You can then separate the sugar that dissolved from the rice that didn't by simple filtration. In a similar manner a certain portion of the coal will dissolve if placed in the proper solvent. The dissolved portion of the coal can be separated from the undissolved portion by filtration or centrifugation. The water can be driven off and the dry sugar recovered in a manner similar to driving off the solvent and recovering the dissolved portion of coal.

The solvent extraction of coal has been employed for many years as a means of studying the constitution of coal and as a coal upgrading technique. Unlike many other coal upgrading processes, solvent extraction utilizes relatively less severe treatment conditions and leaves the greater part of its products similar to the original coal. Attempts have been made in the past to explain various coal properties, coking behavior, rank of coal, degree of oxidation, and so forth, on the basis of solvent action on coals (Gadam 1990).

Bedson, in 1902, discovered that bituminous coals are substantially soluble in hot pyridine (Berkowitz, 1974). Since then, the solvent extraction of coal has been extensively studied. The early studies on solvent extraction were based on the view that coal consists of a mixture of organic substances, including a "coking principle" which was responsible for the good coking properties of certain coals. It was thought that solvent extraction could be used to isolate the "coking principle" and to upgrade the poor 
coking coal. Several solvents have been used in the past for coal extraction viz. acetone, vinyl, methyl and ethyl alcohols, aniline, benzaldehyde, benzene and its homologues, pyridine, carbon disulfide, petroleum ether, tetralin, phenol, furfural, ethylene diamine etc. (Lowry, 1945). The voluminous literature on solvent extraction of coal has been reviewed by Bakes (1933), Kiebler (Lowry, 1945), Stoppes and Wheeler (1924), Wise (1971) and Dryden $(1950,1951,1952)$.

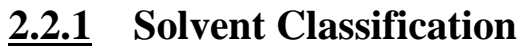

A classification of solvents with respect to their effect on coal was proposed by Oele et al. (1951). Solvents that are conventionally used for coal extraction can be grouped into four general categories:

1) Non-specific solvents extract a small amount of coal (10\%) at temperatures up to $125^{\circ} \mathrm{C}$. These solvents extract the resins and the wax residues in the coal. The extract material is typically aliphatic in nature. Alcohols, benzene, ethers, acetone, and chloroform, are examples of non-specific solvents.

2) Specific solvents are defined these types of solvents that dissolve appreciable quantities of coal (20 to $40 \%$ ) below $230^{\circ} \mathrm{C}$ by a process of physical dissolution (Dryden 1951). Dryden showed that effective specific coal solvents are those that contain a nitrogen atom and an oxygen atom with unshared electrons as a lone pair. This unshared lone pair of electrons tends to affect the solvent polarity and the coal swelling characteristics of the solvent. The nature of the extract is virtually indistinguishable from the original coal. NMP (N-methyl-2-pyrrolidone) is an example of a specific solvent.

3) Degrading solvents extract major amounts of the coal (more than $90 \%$ ) at temperatures up to $425^{\circ} \mathrm{C}$. The solvent can be recovered from solution substantially unchanged. Their action is presumed to depend on mild thermal degradation of coal to produce smaller soluble fragments. Phenanthrene, anthracene oil, etc. are examples of degrading solvents. 
4) Reactive solvents dissolve coal by chemical interaction. The chemical interaction of the solvent promotes degradation of the coal matter during extraction. This causes the solvent to change chemically during the process. Examples of reactive solvents include: low temperature alkaline hydrolysis of coal by alkali alcoholate resulting in the partial depolymerization of the coal matrix, extraction of coals at temperatures above $300^{\circ} \mathrm{C}$ with oleic acid resulting in the formation of asphalt-like material containing combined oleic acid, and the high temperature depolymerization of coal in the presence of hydrogen or hydrogen-donor solvents such as tetralin (tetralin loses hydrogen to form naphthalene) (Philips 1988).

\subsubsection{Coal and Solvent Properties Effecting Extraction}

Several attempts have been made to correlate coal solubility with some physical parameter of either the solvent and/or the coal. Illingworth (1922) observed that when certain coals were heated to 200 to $400^{\circ} \mathrm{C}$ in an inert atmosphere, cooled subsequently and extracted with solvents, the yield was higher than that from untreated coals. Asbury (1934) studied the influence of time on extraction. Asbury found that after the initial extraction period of 32 hours, the relation between time and time-yield was linear and its slope gave the ultimate yield. Keppler and Brochers (1934) found that an increase in temperature increased the extent of extraction. Curless (1963) showed that a smaller size of coal particle produced a higher yield of extraction.

Dryden $(1950,1951)$ reviewed the voluminous literature on solvent extraction of coal. Compiling this review with his own research (1951), Dryden concluded that the coal solubility in the solvent had very little correlation with the internal pressure, surface tension, dielectric constant, or dipole moment of solvent. He distinguished solvents capable of breaking the intermolecular bonds in coal from those that can stabilize coal fragments in solution. Dryden proposed that a family of solvents containing a nitrogen or oxygen atom possessing an unshared pair of electrons were desirable solvents.

Dryden proposed a model in 1955 to explain the process of dissolution based on the assumption that coal consisted of units called micelles. These micelles are a smaller unit of the macerals, which can vary in size over a range of 1 to 300 microns and are assumed to be indestructible. The desired family of solvents mentioned above act by 
swelling the micellar network so that the smaller units of coal could be extracted out of the swelled structure. Dryden substantiated this micellar-sieve extraction mechanism by showing that the extent of extraction was proportional to the extent of swelling. He then stated that a solvent was suitable if it promoted swelling of the coal structure while simultaneously stabilizing the coal micelles in solution. The swelling promotes loosening. The availability of an unshared pair of electrons was considered to be an important factor (Renganathan 1986).

Dryden (1951) has shown that physical properties like surface tension, dielectric constant and dipole moment are of little assistance in classifying solvents for coal extractions. Moreover, the approach of relating physical properties of the solvent to the dissolvability of the solvent is of little value in scientific understanding of the extraction process (Gadam 1990).

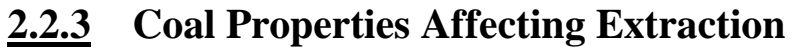

The dissolvability of coal decreases rapidly as the carbon composition of coal increases from 85 to 89 -weight \% (dry ash free basis). The dissolvability then becomes almost negligible at 92 to $93 \% \mathrm{wt} \%$ carbon. This statement applies broadly to all temperatures and types of solvents. Of the petrological constituents, vitrain yields the most extract and fusain the least (Gadam 1990).

\subsubsection{Solvent Nature and Physical Properties Affecting Extraction}

Years of research have been devoted to determining which solvent dissolves what rank, how much, and what proportions of coals. Kiebler put forth a detailed discussion of the effect of the solvent nature (Lowry, 1945). Kiebler's results and can be summed up as follows: "Aromatic solvents are more effective than neutral aliphatic solvents". Extractions with benzene and similar solvents do not peptize the coal, while pyridine extractions do. The acidic or basic nature of aromatic solvents is unimportant although it may affect the yield owing to its contribution to internal pressure (Gadam 1990).

Different solvents can have different types of interactions with the same coal resulting from the specific chemical structure of the solvent. The high solvent power of amines and related solvents for lower rank bituminous coals can be accredited to the 
presence of an unshared pair of electrons on the nitrogen atom (Gadam 1990). Evidence has been obtained showing that the solvent power is closely related to the presence of hydroxyl groups. A two-ring system containing a hydroxyl group attached to an aromatic ring and a hydroaromatic ring was found to be very effective (Dryden, 1950).

The point of whether the solvent action on coal is physical or chemical in nature has always been disputed. It is suspected that certain organic liquids are performing more than a solvent action on coal. An indication of chemical interaction is obtained from the observation that the total weight of the products sometimes exceeds that of the original coal (Lowry, 1945).

The extremely high extraction yield of lignite coals, using etylenediamine as the solvent, is believed to be because of actual chemical reaction of the solvent with the coal (Given et al., 1986). Operating at a temperature below $250^{\circ} \mathrm{C}$ the solvent power appears to be its ability to alter the coal physically (by swelling) prior to the onset of degradation (depolymerization). However, at operating at temperatures above $250^{\circ} \mathrm{C}$, the extraction is accompanied by chemical effects (Dryden, 1950).

Previous experimental results reported in the literature indicate that solvent mixtures enhanced solvent power for certain coals. The possibility of chemical interaction with coal is much higher with the use of mixed solvents (Gadam 1990). However, it has been found that successive (treating the coal with one then another) use of solvents at their boiling points does no better than using the better solvent alone.

\subsubsection{Parametric Study of Solvent Extraction}

The most important parameters that affect the amount of solubility of the coal are time and temperature of extraction. In addition to these two parameters, the particle size of the coal, the presence of moisture and oxygen in the coal, and pretreatment of the coal also affect the solvent extraction of coal to a certain extent. Oxygen degrades most solvents; consequently coal with high oxygen content is not commonly used. The most undesired component of coal is moisture (water). The presence of water in the solvent greatly reduces it solubility. Water can be a real problem, especially in a continuous process where the solvent is continuously recycled causing the water composition to rise decreasing the amount its solubility of coal. 
Some of the important aspects of the parametric study of solvent extraction are discussed below:

\subsubsection{Affect of time and temperature (Kinetics of solvent extraction):}

Several scientists have studied the kinetics of solvent extraction using several different solvents and many different coals. This research focuses on the extraction of the bituminous Upper Powleton coal (WVGS 13421) using N-methyl-2-pyrrolidone (NMP) as the solvent.

Phillips (1988) proposed first order irreversible kinetics for the extraction of Upper Eagle coal using NMP as the solvent and obtained a good fit of the initial rate data with this kinetic model. Philips also investigated NMP extraction of ten different coals and concluded that:

- NMP extraction yields for the ten coals range from 22.8\% (MAF) to $57.9 \%$ (MAF). Coals obtained from the Northern West Virginia coalfield tended to have a higher NMP extraction yields than those obtained from the southern West Virginia coalfield.

- The NMP extraction mechanism is believed to be limited by mass transfer and for temperatures less than $135^{\circ} \mathrm{C}$ due to decreased pore dimensions and availability of active sites for extraction. At temperatures greater than $135^{\circ} \mathrm{C}$ the extraction is limited by mass transfer effects only due to increased swelling of the coal in this temperature region. The increased swelling of the coal at these temperatures exposes more active extraction sites to the solvent resulting in increased extraction rates and yields.

- NMP solvent extraction tends to decrease the BTU value, carbon, and hydrogen content of the residue while it increases the BTU value, carbon, and hydrogen content of the extract.

- NMP solvent extraction tends to increase the total sulfur and ash in the residue and decrease the total sulfur and ash of the extract.

- The activation energy obtained for the high-temperature extraction region (T > $135^{\circ} \mathrm{C}$ ) was $1.3 \mathrm{Kcal} / \mathrm{mol}$ and indicates that the extraction mechanism is controlled by diffusion alone in this temperature region. This value is in good agreement with predicted activation energies for diffusion in liquids.

- The initial rate of the NMP extraction and the ultimate extraction yield increases with increasing treatment temperature. At temperatures greater than $150^{\circ} \mathrm{C}$ the extraction is essentially complete after twenty minutes. 
Phillips observed that with increasing temperature the extraction yield leveled off at a higher value after a period of time. A similar observation was made by Renganathan (1986). Renganathan studied the NMP extraction of Bakerstown coal and found that after about 10 minutes of extraction time, the yield of extract leveled off. He observed a pseudo-first order kinetic behavior of Bakerstown coal and again fitted initial rate data. Gadam (1990) derived a $1^{\text {st }}$ order reversible kinetic rate model to explain the entire kinetics.

\subsubsection{Effect of particle size on extraction Yield:}

Researchers have noticed a significant increase in the extraction yield with the decrease in coal particle size to a certain extent. However, Kiebler considered that the rate of increase in yield with decrease in coal particle size is much too small to be accounted for on the assumption that a surface layer of constant thickness is completely extracted (Lowry, 1945). Renganathan (1986) studied the effect of coal particle size from 60 to 200-mesh on the extraction yield and found the results to be insignificant. This finding is very important from the process economics point of view, since with the conventional crushers coals can be crushed down to 60 mesh size and no grinding of coal (an expensive unit operation) would be required for this solvent extraction process (Gadam 1990).

\subsubsection{Effect of oxygen and moisture on extraction:}

The presence of oxygen during extraction also decreases the yield with most of the solvents (Dryden, 1950). The presence of moisture in coal has an unfavorable effect on extraction yield for solvents that are miscible with water. This is probably because water impedes the power of the solvent since water alone is not a good solvent for coal (Gadam 1990). Water greatly reduces coal's solubility in NMP. As little as 3-mole \% $(0.6 \mathrm{wt} \%)$ water will cut the extraction yield more than $50 \%$. This makes the issue of moisture content in coal extremely important, especially for a continuous process where the solvent would be recycled. 


\subsubsection{Solvent Swelling of Coal:}

Researchers have argued that the ability of a solvent to effectively take the organic matter from the coal into solution largely depends on the degree of penetration of the solvent into the organic matrix pore system. This basically states that a solvent's extraction ability is diffusion controlled. When contacted by a solvent, all but the most mature coals imbibe fluid, swell, and depending on the solvent and extraction conditions, will disperse and dissolve (Phillips 1988).

\subsubsection{Desulfurization of Coal}

The ultimate goal of this research is to produce a clean fuel containing very low ash and sulfur. One of the advantages of using solvent extraction to upgrade coal is that while the extracted coal is in solution it is very amenable to chemical and physical cleaning operations. As discussed earlier, solvent extraction of coal produces an extract almost free of mineral matter.

\subsubsection{Supersolvents}

The term "supersolvents" is used to denote dipolar aprotic solvents that are capable of dissolving a large fraction of organic material in coal (Stiller et al., 1981). As Dr. Alfred Stiller states "Supersolvents have the general formula,

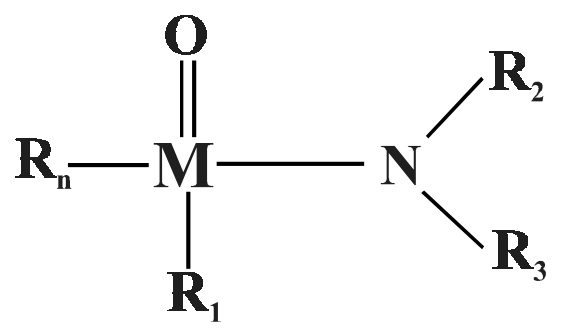

where:

$\mathrm{M}$ is a carbon, sulfur, or a phosphorous atom;

$\mathrm{R}_{2}$ and $\mathrm{R}_{3}$, are each a hydrogen atom or a lower alkyl group;

$R_{1}$ and $R_{n}$ are either each a lower alkyl group, another 

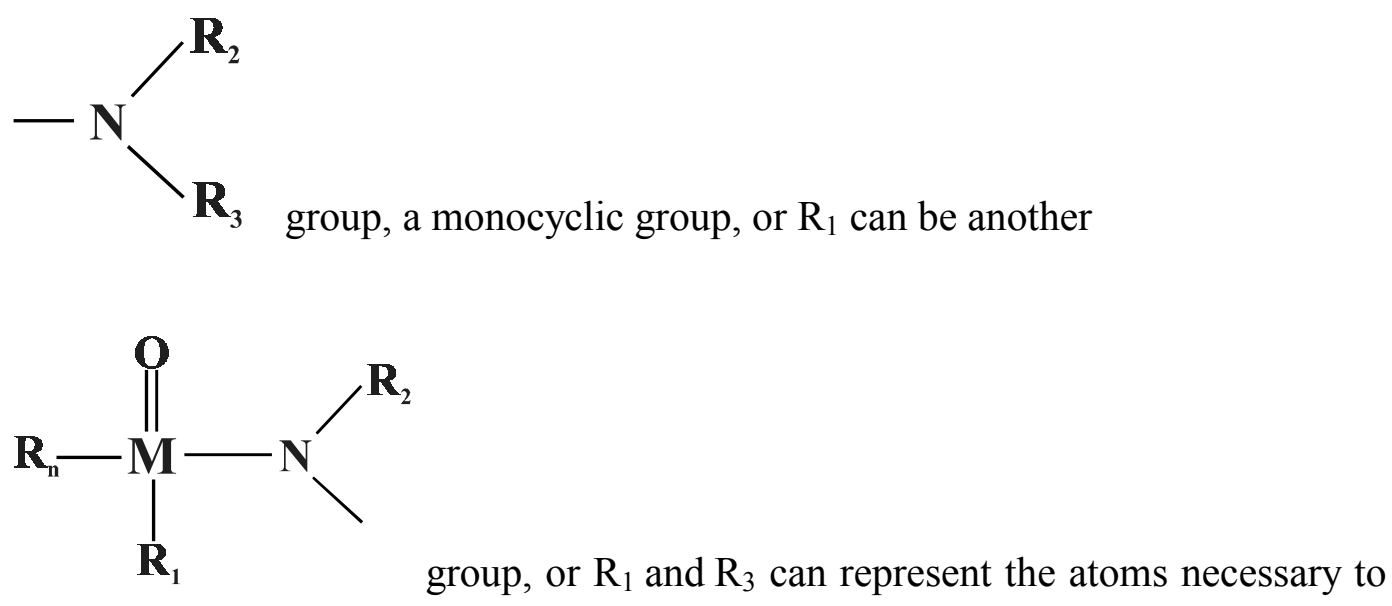
close a heterocylic ring, and $\mathrm{n}=1$ where $\mathrm{M}=$ phosphorus and is otherwise 0 . Where $R_{n}$ and $R_{1}$ are either or both lower alkyl groups in this formula alkyl can apparently have a carbon content in the range of $\mathrm{C}_{1}-\mathrm{C}_{4}$ or possibly $\mathrm{C}_{5}$, of which $\mathrm{C}_{1}$ and $\mathrm{C}_{2}$ are considered preferable. Preferred substituents for $\mathrm{R}_{2}$ and $\mathrm{R}_{3}$ are methyl and ethyl groups, although it is presumed that homologs up to about $\mathrm{C}_{4}$ or possibly higher would produce more or less useful solvent compounds, and the replacement of such groups with one or more hydrogen atoms also appears to be an acceptable alternative. Monocyclic aromatic groups such as benzyl radical might also prove as a benzyl radical might also prove useful as the substituent $R_{n}$ and $\mathrm{R}_{1}$, because the structure of this group is favorable to the resonance stabilizing function of the solvent. Either or both of $R_{n}$ and $R_{1}$ can be another amino group" (Stiller et al., 1981).

Some examples of supersolvents are tetramethyl urea (TMU), dimethyl sulfoxide (DMSO), hexamethyl phsphoramide (HMPA), N-, N-dimethyl acetamide (DMAA), and N-methyl-2-pyrrolidone (NMP) (Stiller et al., 1981).

\subsubsection{Studies of Supersolvents on Coal}

Studies of the effects of supersolvents on coal have shown that they are capable of extracting significant proportions of coal's organic matter at relatively mild extraction conditions. 
Roy et al. (1978) studied the extraction of coal using DMSO as the solvent and found that its solvent capacity is comparable to that of any other good solvent such as pyridine or ethylenediamine.

Leong (1985) and Renganathan (1986) preformed one-hour extraction studies using TMU $\left(166^{\circ} \mathrm{C}\right)$ and NMP $\left(202^{\circ} \mathrm{C}\right)$ at a $10: 1$ solvent-to-coal ratio and obtained extraction yields as high as 50\% (MAF) for TMU and 70\% (MAF) for NMP. NMP has a higher boiling point $\left(202^{\circ} \mathrm{C}\right)$ than either TMU or DMSO. In addition NMP has been shown to swell coal much more than other solvents (Marzec et al. 1979).

Renganathan (1986) investigated NMP's ability to reduce the ash and pyritic sulfur content of coal. Renganathan found that the ash content of various coals could be reduced to as low as $0.1 \%$ by simple extraction with NMP. Renganathan also observed that the pyritic sulfur content of the coal could be significantly reduced by extraction with NMP. The ability of NMP to remove both inorganic material and pyritic sulfur is due to its inability to dissolve appreciably these inorganic species under the mild extraction conditions utilized (Phillips 1988).

Phillips (1988) investigated the extraction yields of 10 different coals using NMP. Phillips concluded that the extraction is essentially complete after twenty minutes when operating above the temperature of $150^{\circ} \mathrm{C}$.

Gadam's (1990) preliminary experiments using NMP as a solvent proved that NMP is a superior solvent to DMSO. Gadam attributed the solvent power of NMP to the presence of the electron pairs on both the nitrogen and oxygen atoms.

\subsubsection{N-methyl-2-pyrrolidone (NMP), The Solvent of Choice?}

Stiller et al. (1985) and Renganathan (1988) used NMP as the solvent of choice for coal extraction experiments at West Virginia University. Through their experiments they concluded that NMP gives higher extraction yields than HMPA, TMU and DMAA. The research proposed here was based upon the results from previous research results. 
The advantages of using NMP as a solvent for coal extraction include: [Leong (1985), Stiller et al. (1985), Renganathan (1986), and Phillips (1988)]

1) High extraction yields $(65+\%)$ are possible at relatively mild conditions $\left(202^{\circ} \mathrm{C}\right.$ and atmospheric pressure) depending on the coal.

2) NMP extraction removes almost all the mineral matter and pyritic sulfur and part of the organic sulfur and during the extraction.

3) Solvent and extract can be easily separated from the coal solution by precipitation with water.

4) The solvent can be reused repeatedly.

5) NMP is a relatively safe solvent for use in coal extractions compared to the other classic coal solvents like: dimethyl sulfoxide (DMSO), hexamethyl phsphoramide (HMPA), N-, N-dimethyl acetamide (DMAA).

The important chemical and physical properties of NMP are shown in Table 2-3 (GAF 1972). Material safety data sheet for NMP, as supplied by the manufacturer BASF is shown in Appendix $\mathrm{C}$ along with other important chemical and physical properties.

Marzec et al. (1979) used the electron donor-acceptor theory to explain the effectiveness of NMP as a solvent for coal extraction. Marzec et al. modeled solvent extraction of coal as a series of replacement reactions of the coal-coal electron donoracceptor bonds by the coal-solvent electron donor-acceptor bonds (Gadam 1990). This theory suggest that solvents with electron donor number $\left(\mathrm{D}_{\mathrm{N}}\right)$ greater than 19 and electron acceptor number $\left(\mathrm{A}_{\mathrm{N}}\right)$ less than 21 would be effective solvents for extraction. NMP has $\left(D_{N}\right)=27.3$ and $\left(A_{N}\right)=13.3$. This model can explain why NMP is an effective solvent. The strong solvating characteristic of NMP on various polar and nonpolar species is also believed to be due to the presence of lone electron pairs on the nitrogen and the oxygen atom (Gadam 1990). Furthermore, the NMP molecule is configured so that it changes its conformation depending upon the relative polarity of the surroundings. This change in configuration also changes the position of the lone pair electrons, which allows the solvent molecule to adapt its polarity to the polarity of its surroundings. This ability of the NMP molecule to adapt itself to its surroundings makes it an extremely powerful solvent for coal extraction where both polar and nonpolar species are encountered (Gadam 1990). 


\section{Table 2-3 Key Physical and Chemical Properties of N-Methyl-2-Pyrrolidone}

Structure:

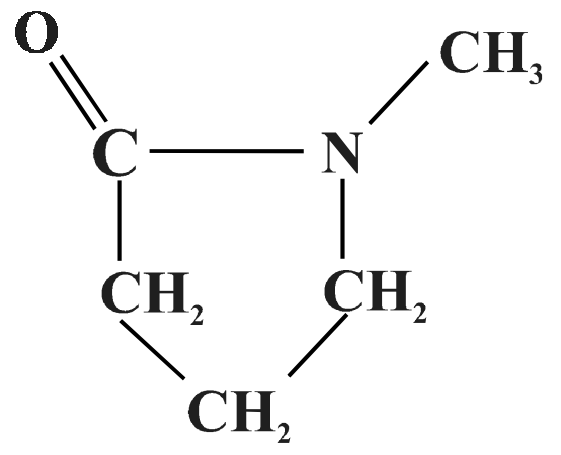

Empirical Formula:

Molecular Weight:

Color (APHA):

Melting Point:

Boiling Point:

Viscosity $\left(25^{\circ} \mathrm{C}\right)$

Specific Gravity:

Specific Heat (Cp):
Physical form:

$\mathrm{C}_{5} \mathrm{H}_{9} \mathrm{NO}$

99.13

Liquid with mild amine-like odor.

50

$-24^{\circ} \mathrm{C}\left(-11.9^{\circ} \mathrm{F}\right)$

$202^{\circ} \mathrm{C}\left(395^{\circ} \mathrm{F}\right) @ 760 \mathrm{mmHg}$

$150^{\circ} \mathrm{C}\left(302^{\circ} \mathrm{F}\right) @ 162 \mathrm{mmHg}$

$100^{\circ} \mathrm{C}\left(212^{\circ} \mathrm{F}\right) @ 24 \mathrm{mmHg}$

$$
\mathrm{T}^{\text {sat }}=\left\{\ln \left(\mathrm{P}^{\text {sat }}\right)+0.2349\right\} / 0.0156
$$

$\left(\mathrm{T}^{\text {sat }}\left[{ }^{\circ} \mathrm{C}\right], \mathrm{P}^{\text {sat }}[\mathrm{mmHg}]\right)$

$1.65 \mathrm{cp}$

$1.027 @ 25^{\circ} \mathrm{C}$

$.987 @ 75^{\circ} \mathrm{C}$

$.969 @ 100^{\circ} \mathrm{C}$

$0.40 \mathrm{Kcal} / \mathrm{kg}$ at $20^{\circ} \mathrm{C}$

$$
\mathrm{Cp}_{\mathrm{NMP}}=8.04 * 10^{\wedge}-4 *(\mathrm{~T})+.38
$$$$
\left(\mathrm{Cp}\left[\mathrm{cal} / \mathrm{gm}^{* \circ} \mathrm{C}\right], \mathrm{T}\left[{ }^{\circ} \mathrm{C}\right]\right)
$$

Thermal Conductivity $\left(\mathrm{k}_{\mathrm{NMP}}\right): \quad \mathrm{k}_{\mathrm{NMP}}=-1 * 10^{\wedge}-4(\mathrm{~T})+0.1954$

$$
\text { (T } \left.\left[{ }^{\circ} \mathrm{C}\right], \mathrm{k}_{\mathrm{NMP}}\left[\mathrm{W} / \mathrm{M} /{ }^{\circ} \mathrm{C}\right]\right)
$$

Heat of Vaporization

Interfacial Surface Tension $\left(25^{\circ} \mathrm{C}\right)$ :

Flash Point (open cup):

Dipole Moment

Dielectric Constant $\left(25^{\circ} \mathrm{C}\right)$ :

Solubility parameter $(\delta)$ :

Miscibility with Other Solvents:
$127.3 \mathrm{~K} \mathrm{cal} / \mathrm{kg}$ at $20^{\circ} \mathrm{C}$

40.7 dynes/cm

$95^{\circ} \mathrm{C}\left(204^{\circ} \mathrm{F}\right)$

4.09 \pm 0.04 Debye

32.2

11.0

completely miscible with water and most organic solvents including alcohols, esters, ketones, aromatic and chlorinated hydrocarbons and vegetable oil. 


\subsubsection{What is the Product of Solvent Extraction of Coal?}

Solvent extraction of coal produces two materials - the extract and residue that can be generally characterized as:

$\begin{array}{llll} & \text { Extract } & & \text { Residue } \\ \text { Organic Material } & \text { All } & & \text { Some } \\ \text { Mineral Sulfur } & \text { None } & & \text { All } \\ \text { Inorganic Sulfur } & \text { None } & & \text { All pyrites \& sulfates } \\ \text { Organic Sulfur } & \text { Some } & \text { some }\end{array}$

Source: Dr.Zondlo 2000

The goal of this research project was to scale up the production of an ash-free coal-based carbon pitch (extract) from the laboratory scale to pilot scale using NMP as the extraction solvent. The product of this solvent extraction is a coal based carbon pitch referred to as "reconstituted coal" or extract. This coal-based product is essentially free of inorganic matter and pyritic sulfur.

The use of different types of solvents and coals produces a variety of coal based carbon pitches. However, supersolvents extraction on bituminous coals produces a relatively consistent coal extract. When the extraction process is complete, and the extracted coal materials are processed to dryness, a "solvent free" solid is produced. Several uses have been identified for this product. Some of the important ones are listed below (Gadam 1990):

1) As an environmentally "clean" fuel, since the ash and sulfur are removed.

2) As a feedstock for further hydrogenation and upgrading to higher quality fuels.

3) As a source of electrode carbon used in the aluminum industry.

4) As a base for varnish manufacture.

5) As a filler in rubber compounding.

6) Addition of coal extracts in noncaking coals to induce caking.

7) Briquette binding.

8) Production of pitch containing little "free carbon".

9) Production of colloidal fuel.

10) Production of low ash carbon.

11) Production of special grades of jet fuels for supersonic aircrafts (Wise1971).

12) Precursor for the production of Carbon Foam.

13) Ingredient to make graphite, coke, binder pitch, and impregnation pitch. 
Zondlo presented a few uses for the extract and its related values at a presentation

to Dupont and New Carbon at Morgantown, West Virginia. These uses and values can be seen in Table 2-4.

\section{Table 2-4 Potential Uses of Coal Extracts}

Products

\section{Fuel}

Low-ash, Low-sulfur

Solid Fuels

\section{High-grade Coke}

Electrodes

Anodes

Graphite

C-C Composites

Pitch

Binder

Impregnation

Mesophase (fiber)

*Source: Dr. John Zondlo 2000
Customers

Power Generation

Transportation

\& Domestic Heating

Steel Industry

Aluminum Industry

Nuclear Reactors

Military and Nuclear

Aluminum \& Steel Industry

Graphite Industry

Nuclear Reactor

Military \& Aerospace
Current Market Price (approx.)

$\$ 3-6 / 10^{6}$ BTU

$\$ 1 / \mathrm{lb}$

$\$ 0.18 / \mathrm{lb}$

$\$ 10 / \mathrm{lb}$

$\$ 10-1500 /$ lb

${ }^{\$} 0.15 / \mathrm{lb}$

${ }^{\$} 0.15 / \mathrm{lb}$

$\$ 1-5 / \mathrm{lb}$ 


\section{CHAPTER 3}

\section{COAL-BASED CARBON PITCH PRODUCTION}

As described earlier, the major effort in this research was the design and construction of a larger pitch production facility to manufacture significant amounts of reconstituted coal. The change in dimension can only be appreciated when consideration is given to the previous laboratory procedure. The following chapter details the previous (laboratory scale) production method, and provides an overview of the scaled-up process (CEPU) with its major steps and equipment. Also the design and construction of the pilot unit is laid out, and the startup is discussed.

\subsubsection{Laboratory Scale Pitch Production}

In the laboratory procedure (shown as a Block Flow Diagram in Figure 3-1), samples of coal are ground to $\leq 60$ mesh $(0.25 \mathrm{~mm})$. After grinding, the coal is dried

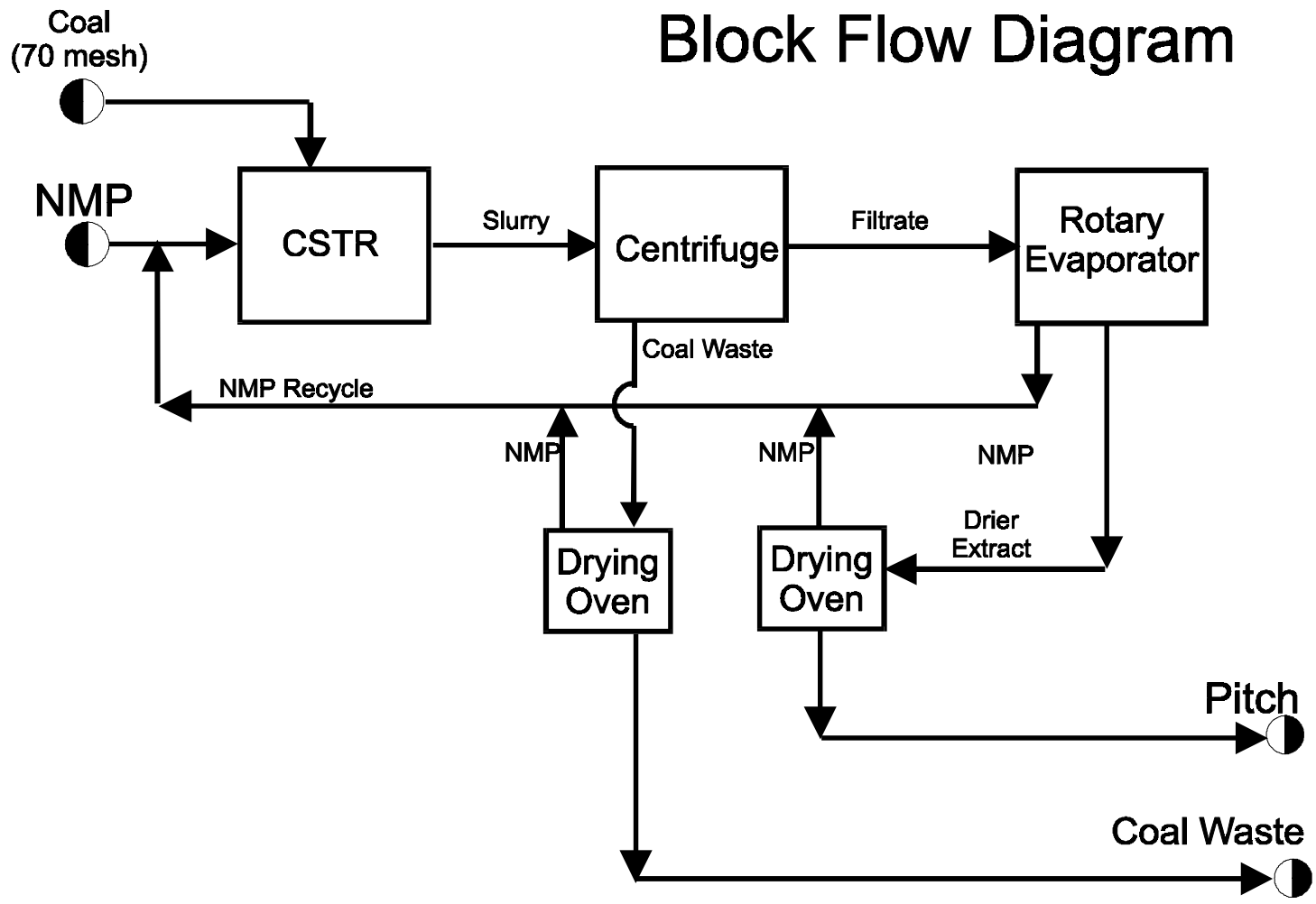

Figure 3-1 Block Flow Diagram for Laboratory Production of Coal-Based Carbon Pitch 
under a nitrogen atmosphere (drying in the presence of air oxidizes the coal and decreases its solubility). The coal is dried because water diminishes the ability of NMP to dissolve coal. The coal is then mixed with the aprotic dipolar solvent, N-Methyl-2-pyrrolidone (NMP), at a ratio of 1 part coal to 10 parts NMP by weight. The coal/NMP slurry is then heated to $202^{\circ} \mathrm{C}$, the boiling point of the solvent. The mixture is kept at this temperature for 1 hour. Prior experimentation has shown that in this time, the maximum portion of the coal is dissolved in the solvent. In a laboratory setting, the dissolution step is done in a 5 gallon stirred tank reactor (CSTR) under a nitrogen atmosphere to prevent any degradation of the solvent. The slurry is then permitted to cool to room temperature. The slurry is then centrifuged to separate the dissolved coal/NMP liquor from the undissolved solid coal/ash. In the laboratory, this centrifugation is done in four symmetrically arranged $750 \mathrm{ml}$ centrifuge containers. It requires 25 minutes at $2000 \mathrm{~g}$ to separate all the ash. After centrifugation, the supernatant containing all the soluble coal material and the NMP is decanted off. The residue that contains all the ash and insoluble coal is removed from the centrifuge flasks and the process is repeated until the 5 gallons of slurry are centrifuged.

The supernatant is then transferred to a rotary evaporator (Rotovap) flask. The rotary evaporator is essentially a batch thin film evaporation unit. While the capacity of the flask is 20 liters, only 8-liter batches of solution can be run at a time. The pressure in the Rotovap is reduced to less than 1-mmHg using a vacuum pump. The flask is rotated in a hot $\left(110^{\circ} \mathrm{C}\right)$ oil bath to heat the mixture. The vapor pressure of the solvent is high enough at this temperature to evaporate off the NMP, which is then condensed using a water-cooled heat exchanger. The condensed NMP is collected for recycle. The evaporation procedure is done until no more condensate (NMP) is given off for $1 / 2$ hour. The required time for evaporation depends upon the dissolved coal-to-solvent ratio. As the solvent is removed, the dissolved coal coats the inside surface of the rotating flask creating a thick skin. This skin reduces the heat transfer rate, thus lengthening the batch time.

After the procedure is completed, the NMP is recovered and recycled back to the reactor for another run. The flask containing the dried coal extract is removed from the Rotovap, filled with a nitrogen atmosphere, and sealed. The sealed flask is put in a 
freezer. At low temperatures the solid coal extract becomes friable and can be "chipped" from the internal surface of the flask. However, at this condition the coal extract is still not completely free of NMP and an additional drying step is needed.

The additional drying step is required to lower the solvent concentration in the coal extract below the level of detection. Once removed, the chipped coal extract is crushed to $\leq 40$ mesh $(0.425 \mathrm{~mm})$ and placed in trays in a vacuum drying oven. The drying oven temperature is set at $205^{\circ} \mathrm{C}$ and the system is evacuated to about $50 \mathrm{mmHg}$. In addition, a low flow rate of preheated nitrogen is permitted to flow over the drying chipped coal extract. The nitrogen carries the NMP out of the oven and into a cold trap installed just before the vacuum pump. This drying process takes over 18 hours. The NMP is again recovered for recycle. The product of this step is the dry coal extract, or pitch, that will be used for subsequent experimentation.

Since NMP is a very expensive solvent $(2.12 \$ / 1 b)$, it is imperative that as much as possible be recovered and recycled. The residue (insoluble coal and ash) from the centrifuge is rich in NMP-nominally it contains about 55\% by weight NMP! NMP is recovered by vacuum drying the residue in the same oven as the extract (described above). This operation also requires about 18 hours. The products of this separation are a high-surface-area coal and NMP. The NMP is again recycled for another run.

Limited by the size and multi-step sequential batch nature of the laboratory preparation process, only a limited supply of coal extract could be prepared. It would take one lab technician 40 hours to make 600 grams of pitch. Therefore, the time required to make $10 \mathrm{~kg}$ of pitch would be greater than 6 months-providing that no equipment failed! The most fragile piece of equipment is the Rotovap. The Rotovap is mostly glass. It is also the most fragile and expensive piece of equipment to fix. Because it is manufactured in Sweden, replacement parts require about 6 to 8 weeks to arrive.

With equipment and personnel limitations, it would be impracticable to proceed with the manufacturing of large quantities of coal extract using the laboratory procedure. A larger experimental operation needed to be designed and constructed to increase efficiency. This facility could also be used to evaluate the economics of the processing steps required for mass production of the coal-based carbon pitch. 


\subsubsection{Coal Extraction Pilot Unit (CEPU)}

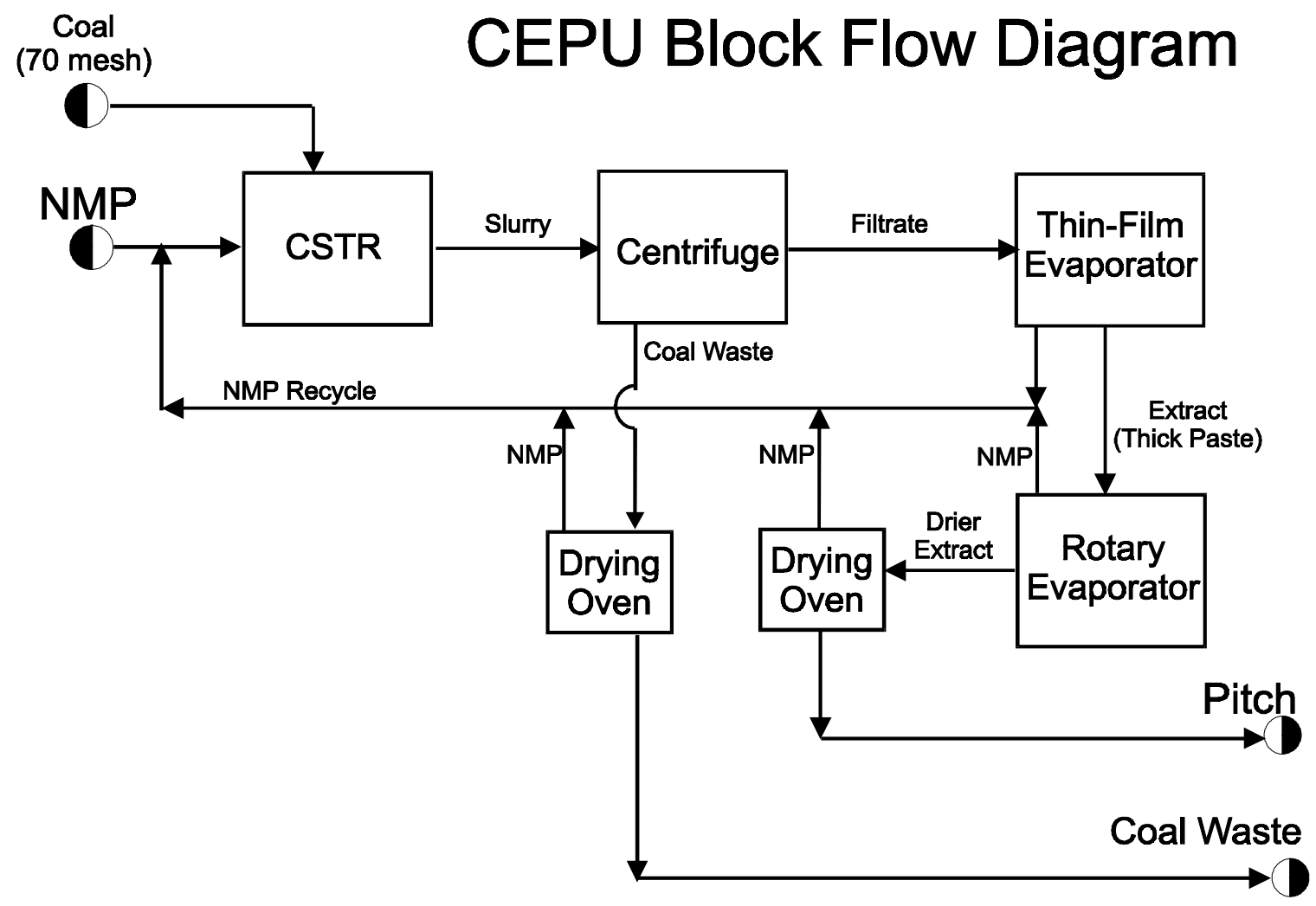

\section{Figure 3-2 Block Flow Diagram for Pilot Plant Production of}

Coal-Based Carbon Pitch

Just prior to the beginning of research on this project, the feasibility of constructing a pilot plant was discussed. The size of the pilot plant equipment would be significantly larger than that used in the laboratory operation. Therefore, more substantial commercial type equipment was to be incorporated into the pilot plant design. A Block Flow Diagram of the pilot plant is shown in Figure 3-2. Instead of a 5-gallon CSTR, a 25-gallon CSTR was to be the extraction unit. Instead of a laboratory centrifuge that had a maximum capacity of four $750 \mathrm{ml}$ flasks and could only reach $2000 \mathrm{~g}$, a small commercial continuous centrifuge that had the capacity of 5 -gallon per minute at $2170 \mathrm{~g}$ was utilized.

In the pilot unit the supernatant, which previously went to the Rotovap for solvent removal, is instead sent to a wiped thin film evaporator (TFE). The TFE removes about $70 \%$ of the NMP at a feed rate of about 0.5 -liter/minute. With a $70 \%$ reduction in 
solvent, the product leaves the TFE as a thick paste. The majority of the remaining NMP is removed from the thickened extract in a modified rotary evaporation unit. The Rotovap takes the remaining 30\% of initial NMP and reduces it down to 5\%. The product leaving the Rotovap contains $\leq 5 \%$ by weight NMP, and is semi-solid. The Rotovap is followed by a vacuum drying step (as described previously) to remove the remainder of the NMP. In each step, NMP is collected and recycled back to the reactor for another run.

This process is essentially the same as the one previously performed in the lab. The primary difference is that $70 \%$ of the solvent is now driven off before the Rotovap by the TFE. A few modifications were made to the Rotovap to increase its performance. The flask is now an old converted 20-liter low-pressure steel Freon tank. With a little machining the tank was made to fit the Rotovap. The larger metal flask facilitates better heat transfer and will permit the drying of larger volumes (14 liters) of thickened sample than the glass vessel. The metal tank also allows more "physical" means of removing the semi-dried extract than the glass. With these modifications, the time required per weight of product is significantly decreased. The drying ovens used in the pilot unit have capacities 30 times greater than the laboratory models. By using commercial type equipment-with some modifications to the Rotovap-the new process is much more efficient than the laboratory process.

The pilot plant can produce up to $35 \mathrm{~kg}$ of pitch in a week under the supervision of two technicians. This is compared to the $0.6 \mathrm{~kg}$ pitch a week in the lab by one technician. The new process allows the production of significant quantities of pitch to be produced in a timely manner. This facility was also used to evaluate the economics of the processing steps required for mass production of the coal-based carbon pitch.

\subsubsection{Construction of the Coal Extraction Pilot Unit}

The construction of a pilot unit was not a simple operation. First, a suitable floor space was required. The Engineering Research Building at WVU had no such space available. The mezzanine floor in the high-bay area in the National Research Center for Coal and Energy (NRCCE) was vacant and suitable. However, this space had neither the power nor plumbing for such a unit. It was agreed that this space would be made 
available with several restrictions. The College of Engineering and Mineral Resources (CEMR) would have to pay for the electrical service installation and the unit had to meet federal safety specifications. Therefore, before any construction could begin, a Chemical Hygiene Plan (CHP) had to be written, reviewed, and approved by the NRCCE safety committee and WVU Environmental Safety and Health (ES\&H) staff. ThE CHP is Appendix A. Once the CHP was approved, the water and electrical requirements were determined and work contracts sent out for bid. After the water, electrical, and safety components were in place, the pilot plant construction was started.

\subsubsection{Pilot Plant Construction Timeline}

Every construction operation needs a timeline so parallel steps can be planned and completed in a timely manner. The construction of the pilot plant was a major operation. Therefore, a timeline was made up to keep everything on schedule.

This project was divided into five main parts. The first part of construction was to design and manufacture the individual components of the CEPU. The second part of the project was to create a detailed Chemical Hygiene Plan to help design the CEPU into an inherently safe working system. The third part was to prepare the laboratory area for the CEPU, and assemble the components. The fourth Part of the research project was to assemble, operate, and debug the system so as to permit convenient production of significant quantities of coal-based carbon pitch. The fifth part of the research project was to generate $15 \mathrm{~kg}$ of coal based carbon pitch for Oak Ridge National Laboratory, and perform a mass balance and energy balance over the system so that some design recommendations could be made.

These five main parts, listed below, are further broken down into tasks. The timeline for each task is shown in the Time Task Deliverable Chart in Figure 3-3.

1) CEPU Equipment Design

Task 1

2) Safety Concerns and Chemical Hygiene Plan

Task 2

3) Mezzanine Preparation and Component Manufacturing \&Assembly

Task $3,4,5,6$

4) CEPU Startup

Task 7,8 ,

5) Preparing the Necessary Extract to Fulfill the ORNL Contract

Task 9,10 \& Material and Energy Balances 
1999-

2000-

Apr. May June July Aug. Sept. Oct. Nov. Dec. Jan. Feb. Mar. Apr. May Task

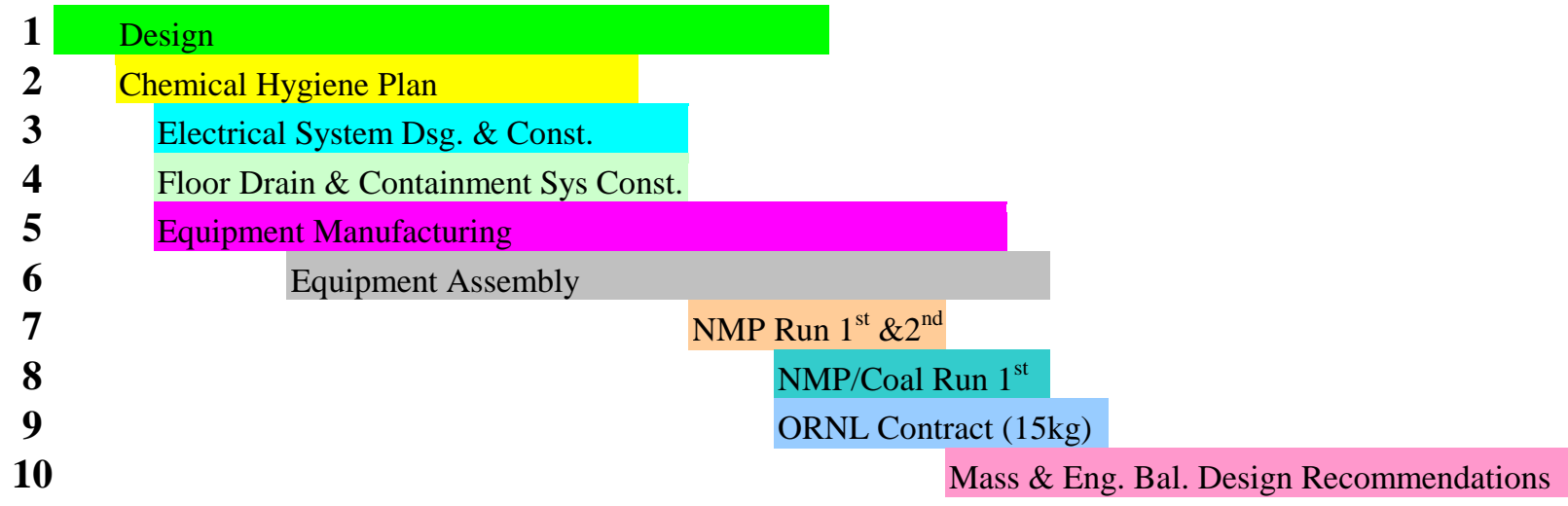

\section{Figure 3-3 CEPU Construction Time Task Deliverable Chart}

\subsubsection{CEPU Equipment Design}

The CEPU equipment can be divided into five main components listed below, as seen on the Block Flow diagram in Figure 3-2. The following five main units and their supporting components were scaled, designed and constructed to handle the $100 \mathrm{~kg}$ of NMP with $10 \mathrm{~kg}$ of coal per run.
1) Continuous Stirred Tank Reactor (CSTR)
(R-101)
2) Centrifuge
3) Wiped Thin Film Evaporator (TFE)
(TFE-101)
4) Rotary Evaporator (Rotovap)
$(\mathrm{RV}-101)$
5) Drying Oven
(DO-101-3)

These main components and their supporting equipment are described in detail in Chapter 4. The components are labeled with equipment numbers that relate to their position on the Process Flow Diagram (PFD). The PFD for this process can be seen in Figures 3-5\&6. A stream table follows in Table 3-1, and an equipment list in Table 3-2. A Block Flow Diagram (BFD) with stream compositions is also shown in Figure 5-1.

\subsubsection{Safety Concerns and Chemical Hygiene Plan}

The designated location for the construction and operation of the CEPU is the mezzanine floor in the high bay of the National Research Center for Coal and Energy 
(NRCCE) building. In order to locate the CEPU at this site, a chemical hygiene plan (CHP) had to be prepared, reviewed by the safety management staff of the NRCCE and the West Virginia University Environmental Health and Safety office prior to any equipment being moved into the building. Only after an approved CHP was received did the construction of the CEPU begin.

The safety considerations mandated that the CEPU facility be designed and constructed to be inherently safe and nontoxic to the operating personnel and the surrounding environment. To achieve this level of safety, a number of concerns needed to be addressed before construction. Below is a portion of the major safety considerations addressed in the CHP. The entire Chemical Hygiene Plan can be found in Appendix A.

- A containment system was manufactured such that if all the fluids catastrophically left the vessels, no solution would escape the containment structure. It was imperative that a safety factor of two be employed in this regard.

- Flammable storage closets for all solvents and materials used in the pilot plant were purchased and used. A ventilation system that would vent any fumes from the system and the cabinets was constructed, and elephant trunk hoses were affixed to all parts of the system that might produce volatile materials.

- Water systems for safety showers, as well as coolants for various pieces of equipment, were built and drains constructed.

- An electrical supply large enough for the various components of the system was fabricated and built by a certified electrician to meet specific safety regulations. This included a Shunt Trip located next to each exit.

- Special training for correct operating procedures of the overhead crane was given to all personnel who worked on the project.

- An Emergency Spill Kit was purchased, along with the necessary Personal Protective Equipment, such as: lab coats, gloves, hearing and eye protection, dust masks, hard hats ...etc.

- Emergency procedures were detailed along with emergency phone numbers and Material Safety Data Sheets. 


\subsubsection{Mezzanine Preparations and Component Manufacturing Assembly}

The CEPU was constructed on the mezzanine in the following steps:

- Once the CHP was approved, designs of the water and electrical systems were drawn up. The specific electrical requirements for each of the components (pumps, heaters, motors, ...etc.) were needed, along with a detailed layout of the placement of the equipment. Contracts were prepared, sent out on bid, and awarded. WVU employees plumbed the water system. Tri County Electric was hired as the certified electrician to install the electrical wiring to meet all electrical codes.

- Once the electrical work was completed, the NRCCE high bay mezzanine was prepared for CEPU installation. This includes fitting the mezzanine for spill safety, installing a water supply system, eyewash, fire extinguishers, drainage system, and contained ventilation system.

- The spill safety consisted of secondary and tertiary containment structures. The containment systems and additional components were designed, purchased, and installed by the researcher.

- The researcher installed the water supply for use as a cooling medium for condensers and heat exchangers and for the safety shower and eyewash.

- The contained ventilation system was designed and installed by the researcher to handle the pilot plant's needs.

- Each piece of equipment had its own support structure(s) designed and manufactured. Detailed drawings were drafted and delivered to a qualified local machine shop to perform the work. The structures were designed to safely support each unit. The structures are easily disassembled and mobile.

- To save time, the equipment was dry-assembled on the ground floor of the high bay while the mezzanine preparation was taking place.

- Once the site preparation was complete, each unit was moved to the mezzanine by the overhead crane.

- Finally, the wiring and plumbing for each piece was installed connecting the individual units into a safe operating system. This system was named the Coal Extraction Pilot Unit (CEPU). A complete Process Flow Diagram can be seen in Figures 3-5\&6. 


\subsubsection{CEPU Startup}

After the site construction was complete, and the equipment assembled, the CEPU was ready for startup. A large part of the time spent on this research was to operate and debug the system to allow convenient production of significant quantities $(3.5 \mathrm{~kg})$ of coal-based carbon pitch from the CEPU. For this step, each unit of the system was first batch tested using NMP alone. It was then tested using coal and NMP.

\subsubsection{First and Second Run With NMP}

The first two runs were done with just NMP to test for leaks, clean the units, develop pump curves, and rate test flow meters. This trial run helped to yield partial operating parameters for each component of the system up to the TFE. Since no coal was use, no separation in the TFE was needed. Experimentation with the TFE followed these tests when coal was used. The NMP from the initial trial runs was then sent to a Rotary Evaporator where the NMP was cleaned up by distillation to be reused. The correction and improvements made after the trial run are as follows. The designated component identification in parentheses refers to unit number shown in Figure 3-5.

- The reactor was taken out of total reflux by placing a collection flask between the condenser (HE-101) and the reactor. This eliminated the drying of the fresh coal.

- The reflux condenser on the reactor (HE-101) was too small. A new larger condenser was installed.

- The centrifuge developed vibrations. Therefore, the direct piping between the centrifuge and the collection tank (T-100) was replaced with flex piping.

- The Cap on the reactor (R-101) leaked and was fixed.

- $\quad$ TFE feed pump (P-103) was plumbed so that the supernatant can be recycled back to the reactor to increase residence time in the centrifuge.

- Temperature sensors were added on the condenser (HE-101) and reactor (R101) top to monitor the vapor temperature.

- Upright tank (T-104) was installed to better measure the NMP to be sent to the reactor. This tank also helped to monitor the rate at which the TFE was fed. 
- The reactor took over 8 hours to cool from $202^{\circ} \mathrm{C}$ to $100^{\circ} \mathrm{C}$. Therefore, a heat exchanger (HE-104) was designed, constructed, and installed to cool the reactors contents before they are sent to the centrifuge (Ct-101).

- Centrifuge feed and reactor recycle pump (P-101) leaked and was rebuilt.

- Pump P-101 was the only pump that had a RPM controller on it. The curve shown in Figure 3-4 plots the rate vs. rpm.

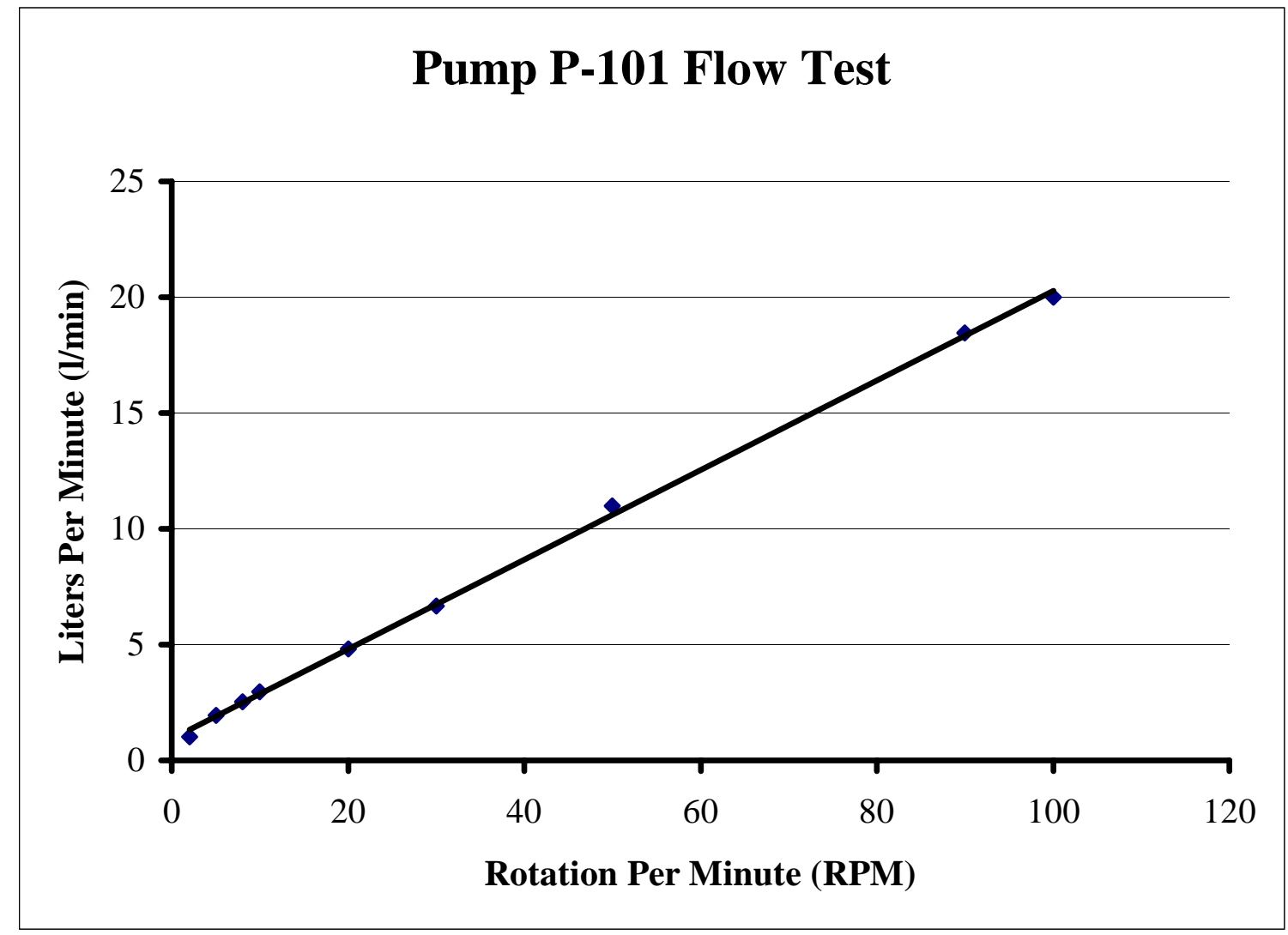

\section{Figure 3-4 P-101 Pump Curve}

\subsubsection{First Run With NMP and Coal}

After the NMP from the first two runs was cleaned, and all improvements and corrections were made to the system, a third run of coal and NMP $(10 \mathrm{~kg}$ coal $/ 100 \mathrm{~kg}$ NMP) was loaded into the CSTR. This run helped to develop more accurate operating procedures and parameters. Once the first coal/NMP run was completed, the system 
performance was evaluated. Again, corrections and improvements were made. The correction and improvements made after the trial run were:

- Unloading TFE product tank T-102 was difficult. A new, funnel-shaped tank was designed, constructed and installed.

- Thermocouple installed on feed line to TFE to monitor feed temp ( $\left.\mathrm{T}_{\mathrm{in}}\right)$.

- Sight glasses were installed on T-101, 103, and 104.

\subsubsection{TFE Startup}

A Wiping Thin Film Evaporator (TFE) is used as the third stage of the CEPU process for manufacturing reconstituted coal. The solvent (NMP) in the CSTR (R-101) dissolves the coal. The dissolved portion of the coal is separated from the un-dissolved portion in the Centrifuge (Ct-101). The TFE is then used to evaporate the majority (72 \pm $2 \%$ ) of the solvent from the dissolved portion of the coal. Finally a Rotovap is used to drive off the most of the remaining solvent from the product before it is placed in a vacuum drying oven to complete the process.

A Pfaudler Wiping Thin Film Evaporator was purchased for this research project. Communication with the manufacture assured the group that the evaporator would be well suited to the extraction process. Experimental results showed that 10 gallon/hour could be processed through the unit. However, the unit was designed for liquid/liquid separation (separating a volatile liquid from a non or less volatile one). The TFE was not designed, and had not been used to separate a liquid from a solid. Therefore, the unit was designed and set up to only drive off a percentage of the solvent so that the product would still be a fluid and flow like a "thick liquid".

The TFE evaporator system came in two separate units: the first unit is the TFE itself (TFE-101); and the second unit is a heater (HE-102), which provides the necessary heat to the TFE for the solvent evaporation. The $20-\mathrm{kW}$ heater unit came assembled and only needed to be fitted to the evaporation unit. The TFE evaporation system is also assisted by a vacuum pump (VP-101) to reduce the temperature at which the NMP is evaporated.

Again, the TFE evaporation system thickens the filtrate (solvent and dissolved coal) by separating a majority of the solvent through an evaporation process from the 
filtrate. Section 4.3.0 explains the operation of the TFE in greater detail. From section 4.3.0 one can see this is a fairly complicated system. There are five operating parameters for the TFE that affect the quality of the product.

1) The flow rate of filtrate into the TFE $\left(F_{\text {in }}\right)$.

2) The temperature of the filtrate feed to the TFE $\left(T_{\text {in }}\right)$.

3) The temperature of the heating fluid from the supporting heat exchanger $\left(T_{m}\right)$.

4) The film thickness on the wall—controlled by the wipers rotation rate (RPM)

5) The internal pressure that controls the evaporation temperature $\left(\mathrm{P}^{\mathrm{sat}}\right)$.

To simplify the system into a solvable problem, a few parameters were assumed. The operator has direct control over all five of the operating parameters. With these assumptions listed below, the only parameter left is the temperature of the heating fluid from the supporting heat exchanger $\left(\mathrm{T}_{\mathrm{m}}\right)$. Therefore, the temperature at which the operator should set the supporting heat exchanger was determined to be $225^{\circ} \mathrm{C}$. The actual temperature was found to be approximately $206^{\circ} \mathrm{C}$. The details of this solution can be found in section 6.2.1.

1) The inlet temperature of the filtrate feed is the highest allowed by the centrifuge $\left(\mathrm{T}_{\text {in }}=100^{\circ} \mathrm{C}\right)$.

2) The filtrate feed rate is the lowest desired value for the CEPU operating $\operatorname{system}\left(F_{\text {in }}=0.5\right.$ liter/min $)$.

3) The internal operating pressure is the lowest the vacuum pump can achieve $\left(\mathrm{P}^{\mathrm{sat}}=0.5\right.$-psia $)$. This pressure is the saturation pressure for the solvent, which will determine at what temperature the solvent is driven off. Figure 6-5 shows a plot of the natural log of the saturation pressure verse temperature for NMP.

4) The rate of rotation of the wiper blades for the solvent/NMP film will be the same as that used for the water experiment $(\mathrm{RPM}=370)$. The results from the water experiment will be used to get the adjusted thermal conductivity value (k) for the solvent/NMP film. 


\subsubsection{Drying Ovens and Rotovap First Runs}

The CEPU has been described as being too big to be a laboratory-scale unit but too small to be a production plant. Certain pieces of equipment were not available in the size needed for the pilot plant. Therefore, the Rotovap and drying oven from the lab had to be used. Due to the large increase in material processed by each unit, some modifications had to be made.

The Rotovap was not modified significantly. The only major modification was the addition of a steel vessel instead of the glass one as explained in section 4.4.0. The drying ovens had never been used to dry any significant amount of material. Three vacuum drying ovens were utilized by the CEPU. As a result of the large amount of material being processed, a special condensing system (HE-105) was installed for the ovens as described in section 4.5.0.

\subsubsection{Preparing the Necessary Extract to Fulfill the ORNL Contract}

The majority of the support for this project is a contract with Oak Ridge National Laboratory (ORNL) Lockheed Martin Subcontract \# 19X-TA437C. The product of this contract was the manufacture of $15 \mathrm{~kg}$ of spinable coal-derived carbon pitch. Spinable carbon pitch must have no ash. This quantity of pitch could only conveniently be manufactured in a unit like the one constructed for this project. After the operation conditions were established a major effort was expended to fulfill the ORNL contract. 


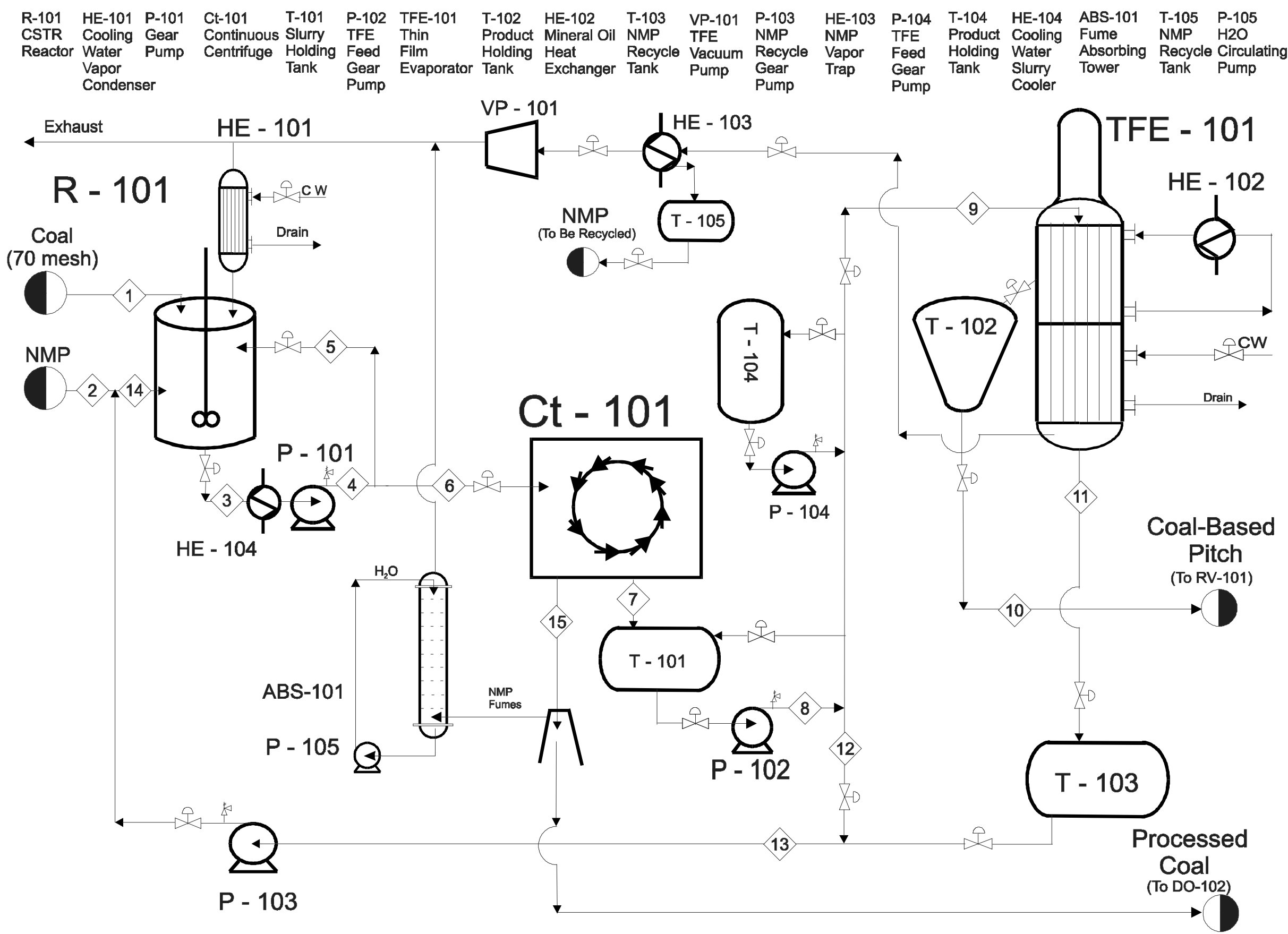

Figure 3-5 Process Flow Diagram for Production of Coal-Based Carbon Pitch 


\begin{tabular}{|c|c|c|c|c|c|}
\hline $\begin{array}{l}\text { RV-101 } \\
\text { Roto-Vap }\end{array}$ & $\begin{array}{l}\text { DO-101 } \\
\text { Pitch } \\
\text { Drying } \\
\text { Oven }\end{array}$ & $\begin{array}{l}\text { DO-102 } \\
\text { Waste Coal } \\
\text { Drying Oven }\end{array}$ & $\begin{array}{l}\text { DO-103 } \\
\text { Waste Coal } \\
\text { Drying Oven }\end{array}$ & $\begin{array}{l}\text { HE-105 } \\
\text { Vapor } \\
\text { Trap }\end{array}$ & $\begin{array}{l}\text { T-106 } \\
\text { NMP } \\
\text { Recycle } \\
\text { Tank }\end{array}$ \\
\hline
\end{tabular}

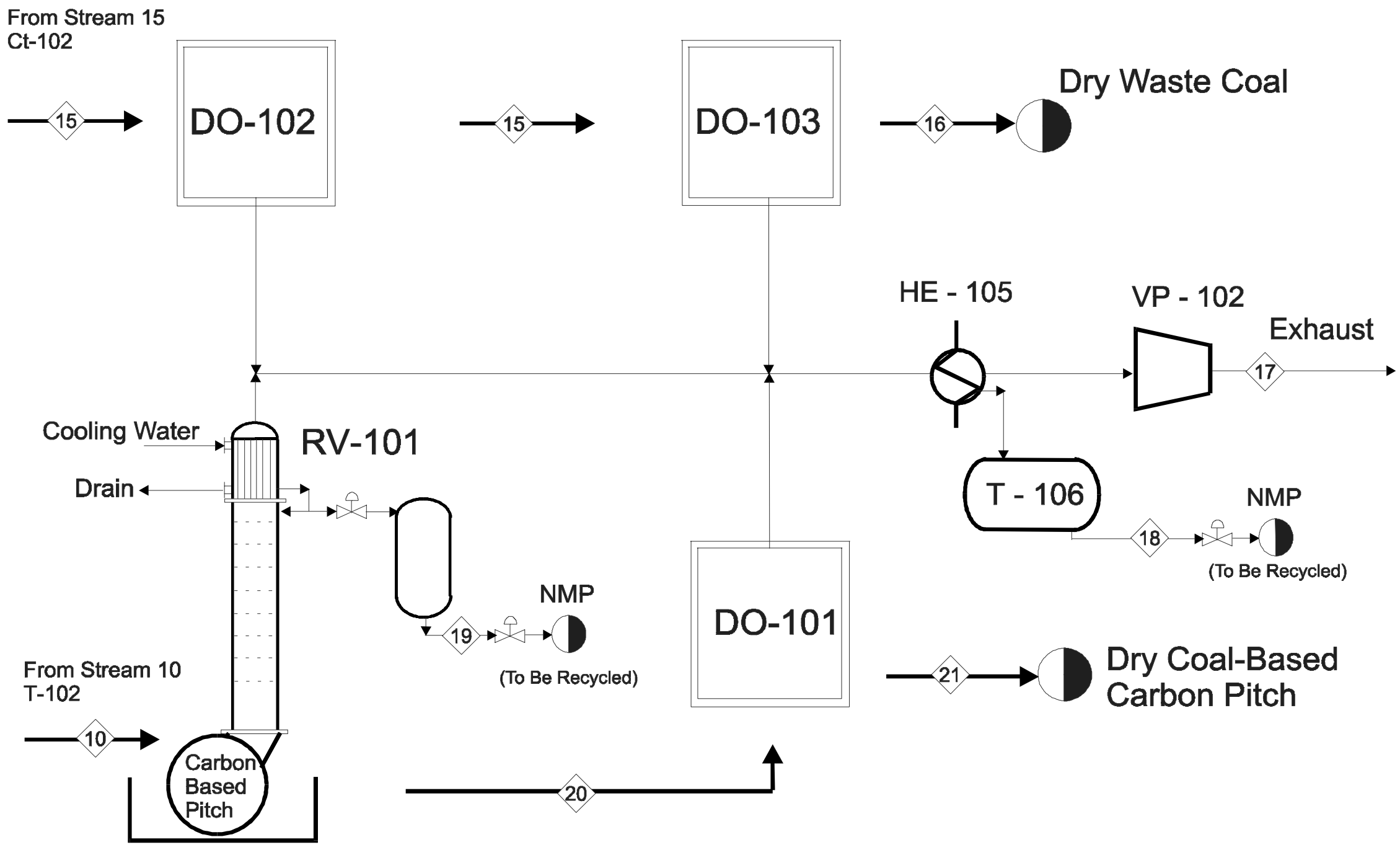




\section{Table 3-2 Coal Extraction Pilot Unit Stream Table}

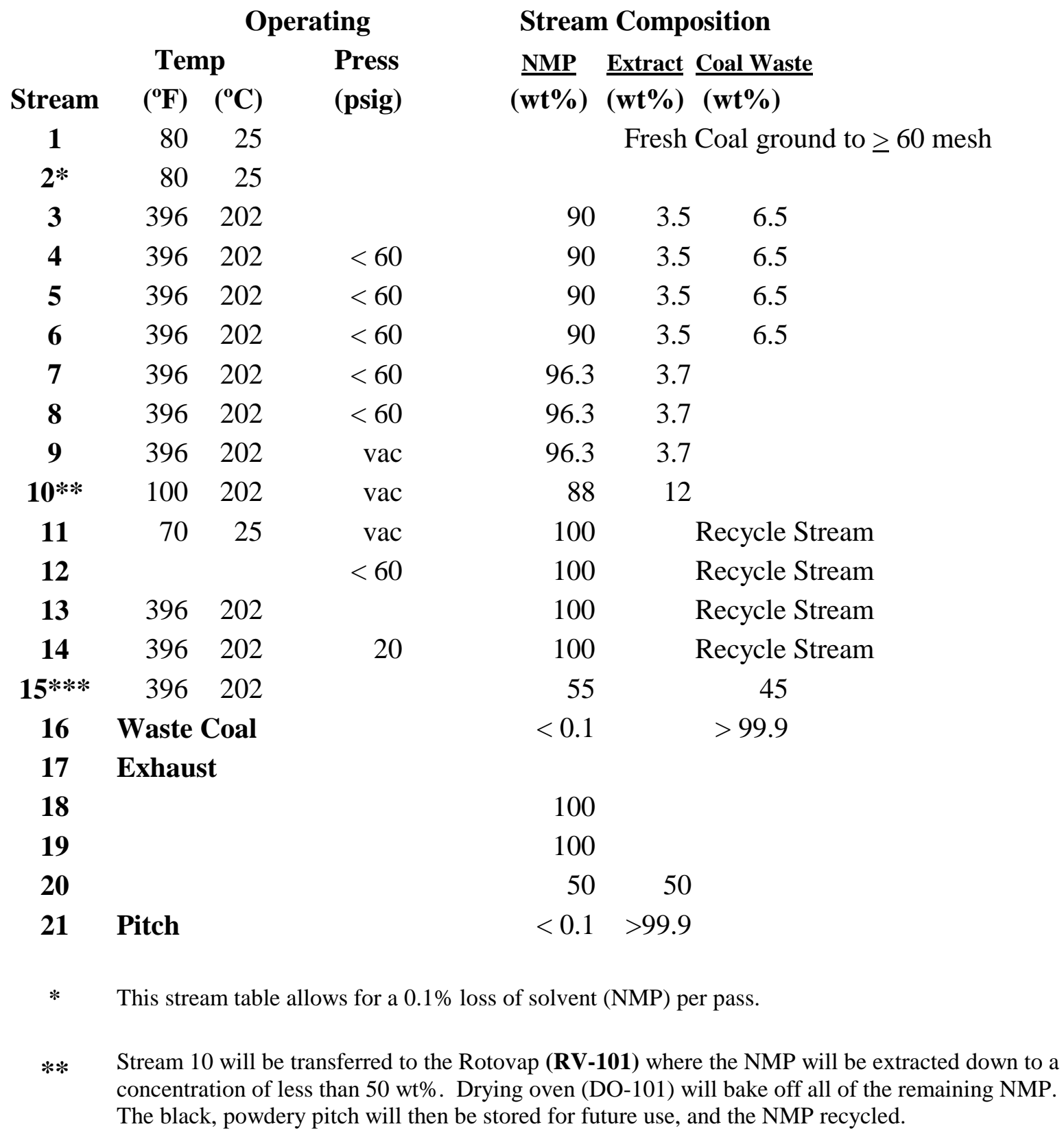

*** Stream 15 will be transferred to drying ovens (DO-102 \& 103) where the remaining NMP will be extracted down to a concentration of less than $1 \mathrm{ppm}$ NMP. The processed coal will then be in powdered form again. The only difference is the used coal will have a higher ash (mineral) content.

No clean up process shown on PFD for the $\mathrm{H}_{2} \mathrm{O} / \mathrm{NMP}$ condensed by the reactor condenser (HE-101). 


\section{Table 3-3 Equipment List for Coal Extraction Pilot Unit}

\section{Equipment List}

\# Discription

CSTR Reactor (with Stirrer \& Heater)

Ct-101 Sharples Penwalt Centrifuge

TFE-101 Pfaudler Wiped Thin Film Evaporator

RV-101 Buchi R-135 Rotary Evaporator

DO-101 NAPCO Model 5961Vacuum Drying Oven

DO-102 NAPCO Model 5961Vacuum Drying Oven

DO-103 NAPCO Model 5961Vacuum Drying Oven

ABS-101 NMP Vapor Absorbing Tower

HE-101 Reactor NMP/ $\mathrm{H}_{2} \mathrm{O}$ Vapor Condenser

HE-102 Heater for TFE (Mineral Oil)

HE-103 Vapor Trap/Condenser for VP-101

HE-104 Reactor Slurry Cooler before Centrifuge

HE-105 Drying Oven Condenser \& VP-102 Vapor Trap

P-101 Tuthill Gear Pump Model 25A D.I. 0.5

P-102 Tuthill Gear Pump Model 5A D.I.

P-103 Magnetic Drive NMP Recycle Pump $\quad 0.125$

P-104 Tuthill Gear Pump Model 25A D.I.

P-105 Absorber $\mathrm{H}_{2} \mathrm{O}$ Recycling Pump (Centrifugal) 0.5

VP-101 Vacuum Pump for TFE

VP-102 Vacuum Pump for Drying Ovens \& Rotovap 1

T-101 Centrifuge Product Tank NMP/Disssolve Coal)

T-102 TFE Product Tank (NMP/Dissolved Coal)

T-103 Tank for Condensed NMP from TFE

T-104 Upright Measuring Tank

T-105 Tank for VP-101 Condenser / Vapor Trap

T-106 Condenser Tank for Vapor Trap for Drying Oven

\begin{tabular}{ccccr} 
& & & & \\
hp) & (kW) & (Volts) & (Amps) & (Ph \\
\hline 1 & 13 & 220 & 60 & 1 \\
7.5 & & 220 & 21 & 3 \\
3 & & 220 & 7.6 & 3 \\
& 3.2 & 220 & 145 & 3 \\
& 1.5 & 120 & 12.5 & 1 \\
& 1.5 & 120 & 12.5 & 1 \\
1.5 & 120 & 12.5 & 1
\end{tabular}

\section{Vol}

(gal)

3

$\begin{array}{ccc}220 & 65 & 3 \\ 110 & 5 & 1\end{array}$

5

$\begin{array}{lll}110 & 5 & 1 \\ 110 & & 1\end{array}$

\section{0}

220 6.2

220

110

110

110

7.4

11

60

30

30
60

60

5

20
Surface Area Oper. Press Oper. Temp

Inside|Outside Max $\mid$ Norm

$\left(\mathbf{f t}^{2}\right)$

$\left(\mathrm{ft}^{2}\right)$

(psia)

\begin{tabular}{lc} 
(psia) \\
\hline 9.6 & 14.6
\end{tabular}

1.2

1.3
6.5

(14.

$295<0.2<650-408$

$14.6<0.2 \quad 356 \quad 230$

$14.6<0.2 \quad 500 \quad 410$

$14.6<0.2 \quad 500 \quad 410$

$14.6<0.2 \quad 500 \quad 410$

$14.6 \quad 14.6$

80

$\begin{array}{cc}500 \quad 396 \\ 600 & 408\end{array}$

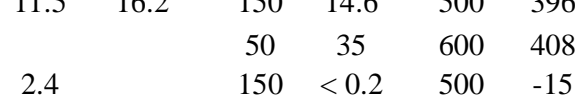

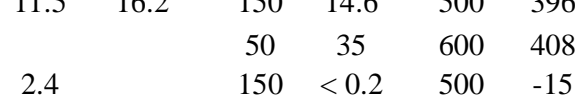

Flow Rate Min $\overline{\text { Max }}$ Norm $(\mathrm{gal} / \mathrm{min})(\mathrm{gal} / \mathrm{min})(\mathrm{gal} / \mathrm{min})$

$\begin{array}{lll}0 & 5 & 1\end{array}$

$\begin{array}{lll}0.2 & 2 & 0.5\end{array}$

$5 \quad 36 \quad 15$

$\begin{array}{cccccc}7.3 & 7.85 & 60 & 20 & 600 & 396 \\ 15.5 & 19.6 & 75 & <0.2 & 400 & -15\end{array}$

$200 \quad 20 \quad 525 \quad 396$

$200 \quad 20 \quad 525 \quad 200$

$25 \quad 5$

$200 \quad 20$

$40 \quad 30$

$14.6<0.2$

$14.6<0.2$

$120 \quad 14.6$

$120<0.2$

$120<0.2$

$120 \quad 14.6$

$120<0.2$

$120<0.2$ 


\section{CHAPTER 4}

\section{COAL EXTRACTION PILOT UNIT}

\section{Prelude}

As described in Chapter 3, the Coal Extraction Pilot Unit can be divided into 5 steps. In this chapter a detailed explanation is given of the major pieces of equipment and the supporting components associated with them. The designated component identification labels in parentheses, both below and in the following figures, refer to unit number shown in Figure 3-5.

The first step of the process is the dissolution step. The equipment in this step is: (1) the Continuously Stirred Tank Reactor (R-101), (2) the reactor condenser (HE-101) (3) the reactor recycle and centrifuge feed pump (P-101).

The second step of the process is the separation of solubles (the portion of coal that dissolved) from the insolubles (the coal and ash material that didn't dissolve). This is done by centrifugation. The components described in this chapter for this step are: (1) the centrifuge (Ct-101), (2) the heat exchanger used to prepare the extract mixture before centrifugation (HE-104), (3) the absorption tower for NMP vapors in the exhaust system (ABS-101), (4) the collection tank (T-101), and (5) upright measuring tank (T-101).

The third step is the partial evaporation of NMP from the filtrate by the Wiped Thin Film Evaporator (TFE). The components of this step described in this chapter are: (1) the TFE (T-101), (2) the supporting hot oil heater (HE-102), (3) the vacuum system (VP-101), (4) vapor trap (HE-103), (5) the product (thickened extract) tank (T-102), and (6) the condensed solvent collection tank (T-103).

The fourth step takes the thickened extract from the TFE and dries it to a semisolid using a Rotary Evaporator. The components in this step described in this chapter are: (1) the Rotary Evaporator or Rotovap (RV-101), (2) supporting vacuum system (VP102), and (3) vapor trap (HE-105).

The fifth step provides the final drying of the semi-solid reconstituted coal from the Rotovap, and recovers the solvent from the undissolved coal discharged from the centrifuge. Three drying ovens accomplish the final drying. The components in this 
step described in this chapter are: (1) the drying ovens (DO-101, 2, \&3), (2) the supporting vacuum system (VP-102), and (3) condenser/vapor trap (HE-105).

\subsubsection{Continuously Stirred Tank Reactor (R-101):}

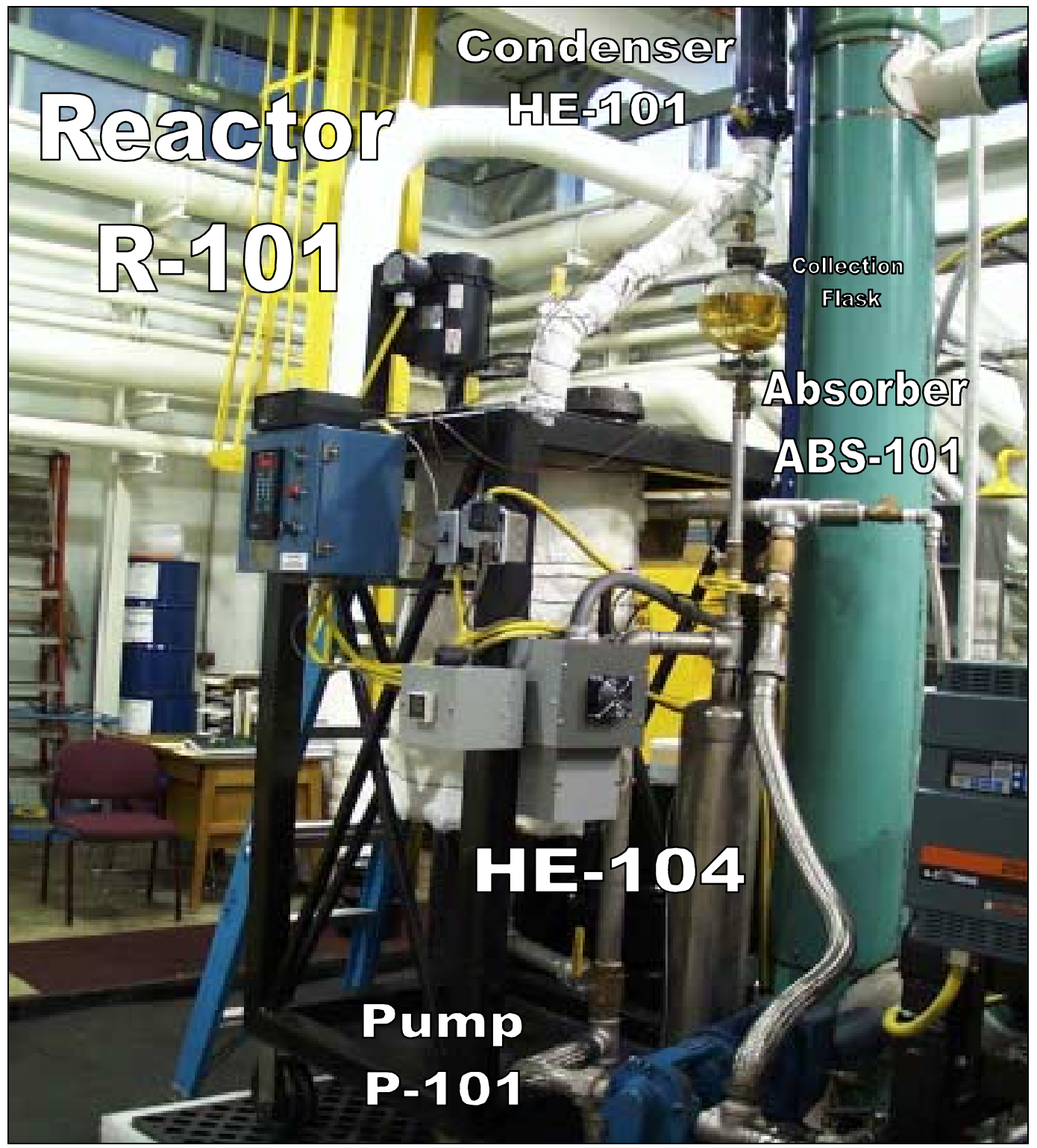

\section{Figure 4-1 Continuous Stirred Tank Reactor with Heat Exchanger, Pump, Condenser, and Absorber}

A suitable batch size for the Continuous Stirred Tank Reactor (R-101) unit shown in Figure 4-1 is 100 liters (26 gallons). The cylindrically shaped reactor vessel is 36 inches high and 18 inches in diameter with a maximum capacity of 40 gallons. The 
bottom of the vessel slopes to a 2-inch Nominal Pipe Thread (NPT) exit nipple. The vessel is supported in a frame 60 inches high with a 32 -inch square top and bottom. The frame is made from 3-inch square tubing with a wall thickness of 1/8 inch. The support structure and vessel are designed so that if more volume is desired, a larger vessel (100gal maximum) can fit directly into the frame. The frame is set on four 6-inch diameter composite wheels so the system can be easily rolled to a suitable position.

The reactor is equipped with several components and features to aid in its operation. Figure 4-2 shows the top view of the reactor vessel. The top of the vessel is sealed with a $1 / 4$ inch steel plate fitted as a flange. This top plate, or lid, is equipped with 6 ports. One of the ports is located in the center of the top plate and houses a shaft that extends down into the vessel. There are four impellers attached to the shaft. The top two impellers direct the flow from the top of the vessel downward and the bottom two impellers direct the flow upward from the bottom of the vessel. This facilitates turbulent flow

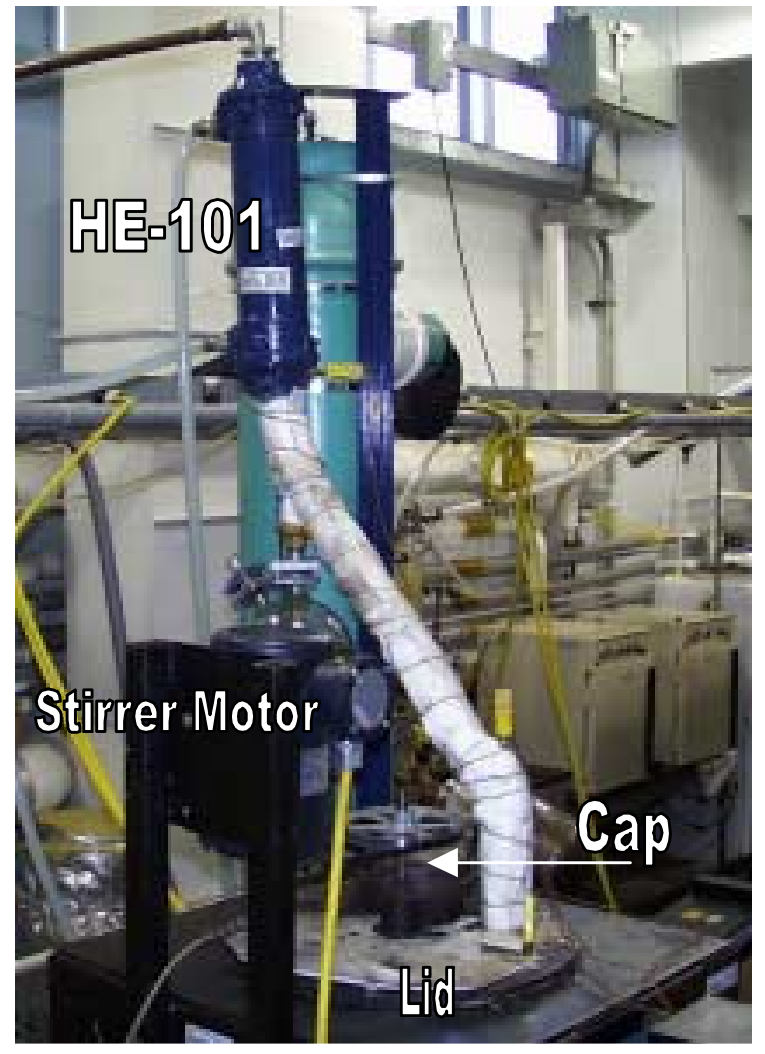

Figure 4-2 CSTR Lid Showing Motor, Cap, and Condenser and provides good mixing. The agitating shaft is driven at 120 RPM by a 1 horsepower electric motor.

Two of the six ports are used to insert thermocouples in the reactor for temperature control and readout. Another port is for the addition of nitrogen. The reactor is kept under a nitrogen blanket to ensure that oxygen does not degrade the NMP at the elevated temperature. The nitrogen is fed into the reactor through a $1 / 4$-inch compression fitting located on the opposite the side of the condenser. Attached to this fitting is a gas line from a nitrogen cylinder. This enables the unit to be maintained under a constant nitrogen blanket. 
The top plate is also is equipped with 2 access ports. One of the access ports is a 6-inch threaded nipple welded into the lid. This nipple is equipped with a cap that can easily be removed to add fresh coal and solvent at the start of a run. The other port is a 1inch collar. Attached to the collar is a water-cooled heat exchanger (HE-101).

A normal batch run consists of 100 liters of NMP mixed with $10 \mathrm{~kg}$ of fresh coal ground to $\leq 60$ mesh $(0.25 \mathrm{~mm})$. The initial slurry fills about $70 \%$ of the total vessel volume; this allows for swelling of the coal particles and the thermal expansion of NMP. The reactor vessel is heated with electric rope heaters. The bottom of the vessel is wrapped with 26 rope heaters. Each rope heater has a capacity of $500 \mathrm{~W}$ yielding a combined $13 \mathrm{~kW}$ heater that draws about 60 amps at 220 volts. Using this heater, the $\mathrm{NMP} /$ coal slurry takes approximately 2 hours to reach its boiling point of $202^{\circ} \mathrm{C}$. Figure 6-2 show the temperature profile for heating the reactor. It is noted that the supporting heater on the reactor is $42 \%$ efficient. This low efficiency is believed to be due to the heat lost in heating the large structural mass of the reactor vessel, and the heat lost by poor insulation.

Once at the boiling temperature, the mixture is then kept at this temperature while stirring for 1 hour. It was found that this one-hour residence time is more than sufficient to dissolve the desired portion of coal. Phillips (1988) found the initial rate of the NMP extraction and the ultimate extraction yield increases with increasing treatment temperature. At temperatures greater than $150^{\circ} \mathrm{C}$ the extraction is essentially complete after twenty minutes. The yield for the Upper Powleton Coal (WVGS 13421) in this research was found to be $36 \pm 2 \%$.

Once the desired portion of coal has been dissolved, the dissolved portion (liquid) must be separated from the undissolved portion (solid). This can be done by centrifugation or filtration. The centrifugation method was chosen for this research. The centrifuge in this research has an upper operating temperature limit off $100^{\circ} \mathrm{C}$. Therefore, the slurry must be cooled prior to centrifugation so as not to exceed equipment operational tolerances. The mixture is cooled to $100^{\circ} \mathrm{C}$ using a single pass shell and tube heat exchanger (HE-104). 


\subsubsection{Reactor Condenser Heat Exchanger (HE-101)}

Heat exchanger (HE-101), shown in Figures 4-1 and 4-2, serves as a condensing unit to condense and collect the water that is driven off the NMP/coal slurry during the heating process. Water inhibits the solubility of coal in NMP and is accumulated in the system from the fresh coal or NMP coming into contact with air. As little as 3-mole \% water will significantly reduce the solubility of coal in NMP. The water and NMP vapors

given off during the heating process are condensed and collected in a flask shown in Figure 4-2. The condensed vapors are collected until the coal/NMP slurry reaches its boiling point of $202^{\circ} \mathrm{C}$. At this point the mixture contains $\leq 0.4$ mole $\%$ water. Once the coal/NMP slurry reaches the boiling point, a valve is switched to allow the condensate from the condenser to flow back into the reactor. This solution in the overhead flask is stored until it can be distilled to remove the water from the NMP. The NMP is then recycled to the reactor for another run.

\subsubsection{Reactor Recycle and Centrifuge Feed Gear Pump (P-101)}

A Tuthill gear pump (P-101) purchased from F.T. Thomas Pump, model number $25 \mathrm{~A} \mathrm{DI}$, is used to circulate the mixture through the tube side of heat exchanger (HE104) to cool the NMP/coal mixture to the maximum operating temperature of the centrifuge. The same gear pump is used to transfer the cooled slurry to the centrifuge where solids (undissolved material or waste coal) are separated from the liquid (NMP and the dissolved coal) at a rate of about 4 liter per minute. This pump is the only one used in the CEPU that has a rate controller. The rate is controlled by the RPM as shown in the plot Figure 3-4. 


\subsubsection{Centrifuge (Ct-101):}

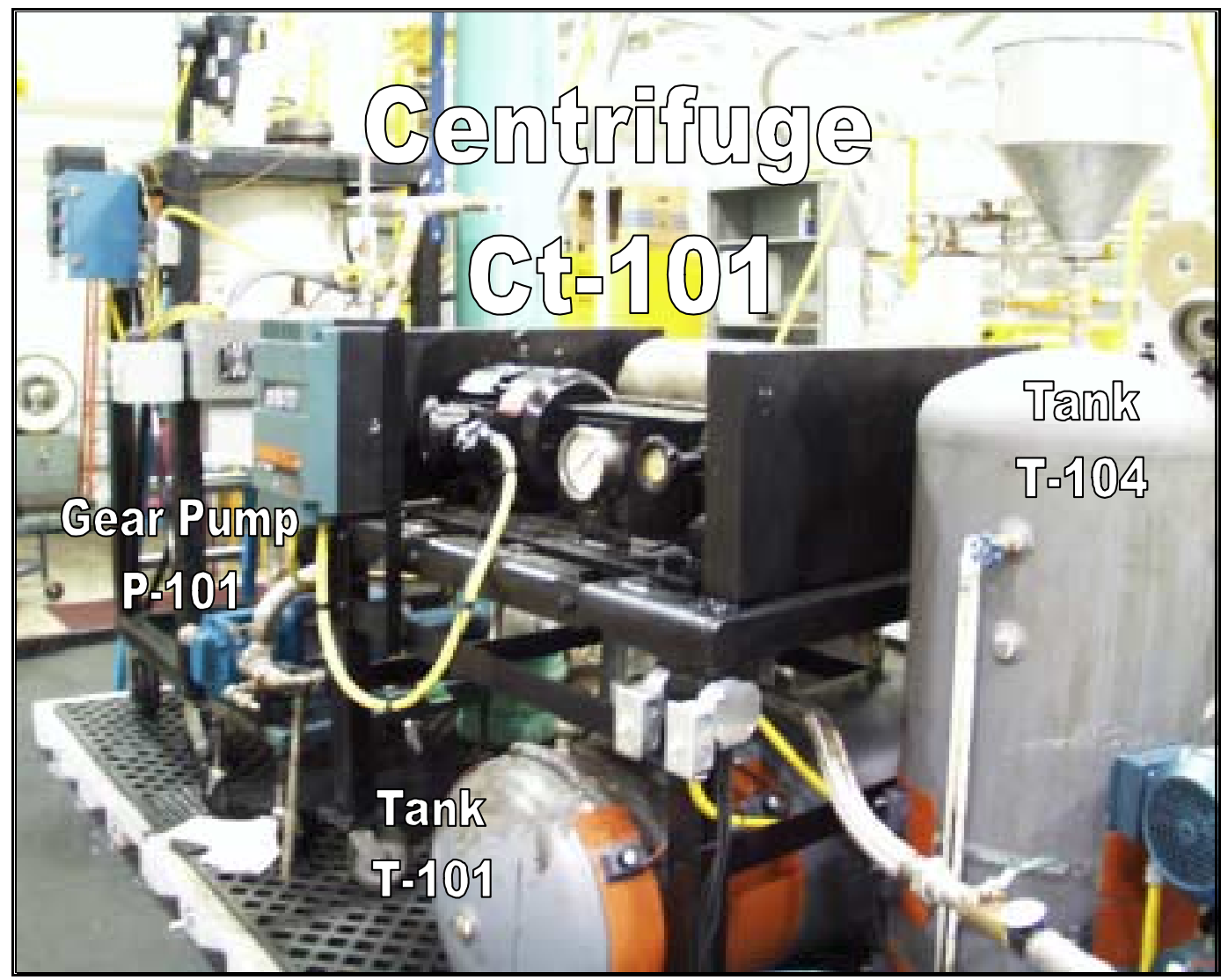

\section{Figure 4-3 Sharples Penwalt BM-PF 290 Centrifuge}

The centrifuge represents an entire step in the process. This step involves several components that combine to perform a set task in the process. The centrifuge used in the CEPU was donated to West Virginia University from Kopper's Industries, Inc.. They previously used it to separate ash and carbon black from molten binder pitch. The unit is a Sharples Penwalt BM-PF 290 shown in Figure 4-3. The unit came without a controller. A suitable controller was purchased and integrated into the unit. Although the maximum RPM rating is 6000, it was found that at 1458 RPM the unit achieved $2200 \mathrm{~g}$ 's. Noting that this is $10 \%$ more than the lab centrifuge, it was felt that this was sufficient to guarantee the desired purity of the extract. This centrifuge has low-temperature seals. As a result, the temperature of the input stream from the reactor must be cooled from $202^{\circ} \mathrm{C}$ to a maximum operating input temperature of $100^{\circ} \mathrm{C}$.

Once the slurry has been cooled to $100^{\circ} \mathrm{C}$, it is fed to the centrifuge by the same pump (P-101) that circulates it through the heat exchanger. The flow rate into the 
centrifuge is about 1 gallon (4 liters) per minute should not exceed its max of 5 gallons (19 liters) per minute. This is a continuous centrifuge where the solid residue is extruded from one side and the filtrate flows from the other end. In this research, the solid residue contains the undissolved portion of the coal as well as ash material. The filtrate (dissolved coal and NMP) flows out the opposite end of the centrifuge and is collected in a 60-gallon tank (T-101) shown in Figure 4-3. This tank is also equipped with a sight glass and heaters so the liquid NMP/dissolved coal batch can be heated before it is sent to the Wiped Thin Film Evaporator. Tank T-101 also has an exit port so samples can be removed for analysis. If the centrifuge fails, and the filtrate contains insoluble material, the sample can be pumped back into the CSTR and centrifuged again. This process could be repeated until the proper purity is obtained.

Once the filtrate meets the desired purity (i.e. ash free) the filtrate is transferred to an upright tank (T-104) by a Tuthill gear pump (P-102), model number 5 A DI, purchased from F.T. Thomas Pump. This containment vessel is a 60-gallon compressed air tank that is vented to the absorbing tower. Due to the fact that tank (T-101) below the centrifuge is in a horizontal position, accurate volume measurements were hard to achieve. As a result, an upright (vertical) tank was installed. The addition of the vertical tank (T-104) makes for more accurate evaluation of the fluid volume. This makes it possible to measure the filtrate volume and predict any solvent loss.

Tank T-104 is also equipped with a sight-glass, so the level of the filtrate can be observed, and heaters so the liquid NMP and dissolved coal batch can be heated before it is sent to the Wiped Thin Film Evaporator.

\subsubsection{Reactor Slurry Cooler Before Centrifuge Heat Exchanger (HE-104)}

As shown in Figure 4-1, located between the CSTR and the centrifuge is a heat exchanger (HE-104). This heat exchanger was designed and manufactured at West Virginia University. It is a stainless steel shell and tube heat exchanger with an internal surface area of $7.3 \mathrm{ft}^{2}$ and an external surface area of $7.85 \mathrm{ft}^{2}$. A gear pump (P-101) circulates the coal/NMP slurry from the reactor through the heat exchanger and back into the reactor until the temperature drops to $100^{\circ} \mathrm{C}$. It takes approximately $1 / 2$ hour of circulating the NMP/coal slurry through the heat exchanger to drop the reactor 
temperature from $204^{\circ} \mathrm{C}$ to $100^{\circ} \mathrm{C}$. Figure 6-4 show the cooling profile for the reactor using the supporting heat exchanger (HE-104). This heat exchanger uses the exit water from the condenser (HE-101) -before it flows to the drain-to lower the slurry temperature of the slurry.

\subsubsection{Absorbing Tower for NMP Vapors}

(ABS-101)

The solid residue or waste coal contains an appreciable quantity of NMP (55\% by weight). This solid residue falls out of the centrifuge and is collected in buckets. NMP has an appreciable vapor pressure at $100^{\circ} \mathrm{C}$. As a result, a light amount of vapor is given off from the waste coal. These NMP vapors are eliminated from the work area by placing a shroud over the residue collection bucket under the centrifuge. The shroud has an elephant trunk that draws the vapors toward the absorber by a ventilation fan, then exhausting the air from the building.

Between the shroud and the ventilation fan is an absorbing tower (ABS-101). The absorbing tower is 11 feet high. It is constructed from a piece of 12-inch SDR-35 PVC pipe. Jaeger Cascade Mini-Rings fill $6.5 \mathrm{ft}$ of the tower. The NMP vapors collected by the shroud are pulled through the absorbing tower by the exhaust fan. These vapors enter the bottom of the absorber just below the Jaeger Cascade ring support. The vapors are pulled up through 6.5 feet of water-saturated rings by the exhaust fan.

The rings are saturated by water flowing over them from the top of the tower. At the bottom of is a 15-gallon collection basin filled with water. A centrifugal pump (P105) circulates water to the top of the absorber. Just below the gas exit port, the water is sprayed through a shower nozzle down through the rings. The water spray wets the plastic hoops and flows downward counter-current to the vapor stream. As the vapor leave the shroud, it passes over the wetted hoops and the NMP vapors are absorbed. Therefore, NO NMP VAPORS ARE RELEASED to the work area or the environment.

The NMP concentration in the absorber is only a trace. It would take over 20 runs to acquire an appreciable amount. Once an appreciable amount of NMP is contained in the water, it can be removed and the water distilled off. The NMP vapors that were absorbed during this research are very minute $(<0.01 \%)$ when compared to the total 
volume. Recovering this small amount of NMP for the water was not worth the effort. Therefore, this microscopic loss of NMP was ignored for this research.

\subsubsection{NMP/Dissolved Coal Collection Tank T-101}

It is important to keep track of the solvent for the material balance over the CEPU. The solvent can be one of four places after the centrifuge: (1) in the filtrate (NMP/dissolved coal) tank (T-101) under the centrifuge, (2) in the condenser collection flask above the reactor (HE-101), (3) in the waste coal, or (4) in the water of the absorber. However the amount of NMP absorbed by the absorbing tower is negligible.

A good approximation of the solvent (NMP) losses could be made when the volume of the filtrate is measured in the vertical tank (T-104). The volume of the NMP and water collected in the collection flask above the reactor is measured using the scales. The difference will tell the amount of NMP entrained in the waste coal.

The residue, or waste coal, leaving the centrifuge is wetted with NMP. This stream of undissolved coal and ash has the consistency of damp potting soil. Therefore, there is some NMP contained in this residue. This waste coal will be dried, and the NMP recovered, weighed and recycled.

\subsubsection{Measuring Tank T-104}

The addition of the upright tank (T-104) increased the accuracy of the material balance. Tank T-104, as shown in Figure 4-3 is calibrated using water and gradations were put on a sight glass to measure the volume easily. Filtrate from the horizontal vessel (T-101) under the centrifuge is pumped into the vertical vessel and its volume is recorded. The vertical vessel also serves as a storage vessel so more than one run of the reactor can be done in a workday, and two runs can be processed through the TFE at the same time.

The NMP in the condenser above the reactor (containing water and NMP distilled off the slurry) will be distilled and reused. The NMP entrained in the waste coal will be recovered by drying the residue in drying ovens (explained later). The NMP in the filtrate will be driven off, condensed, and recycled in the next three steps. Notice that the NMP is always recycled to the reactor to be used again. Solvent recovery is important. 


\subsubsection{Wiped Thin Film Evaporator:}
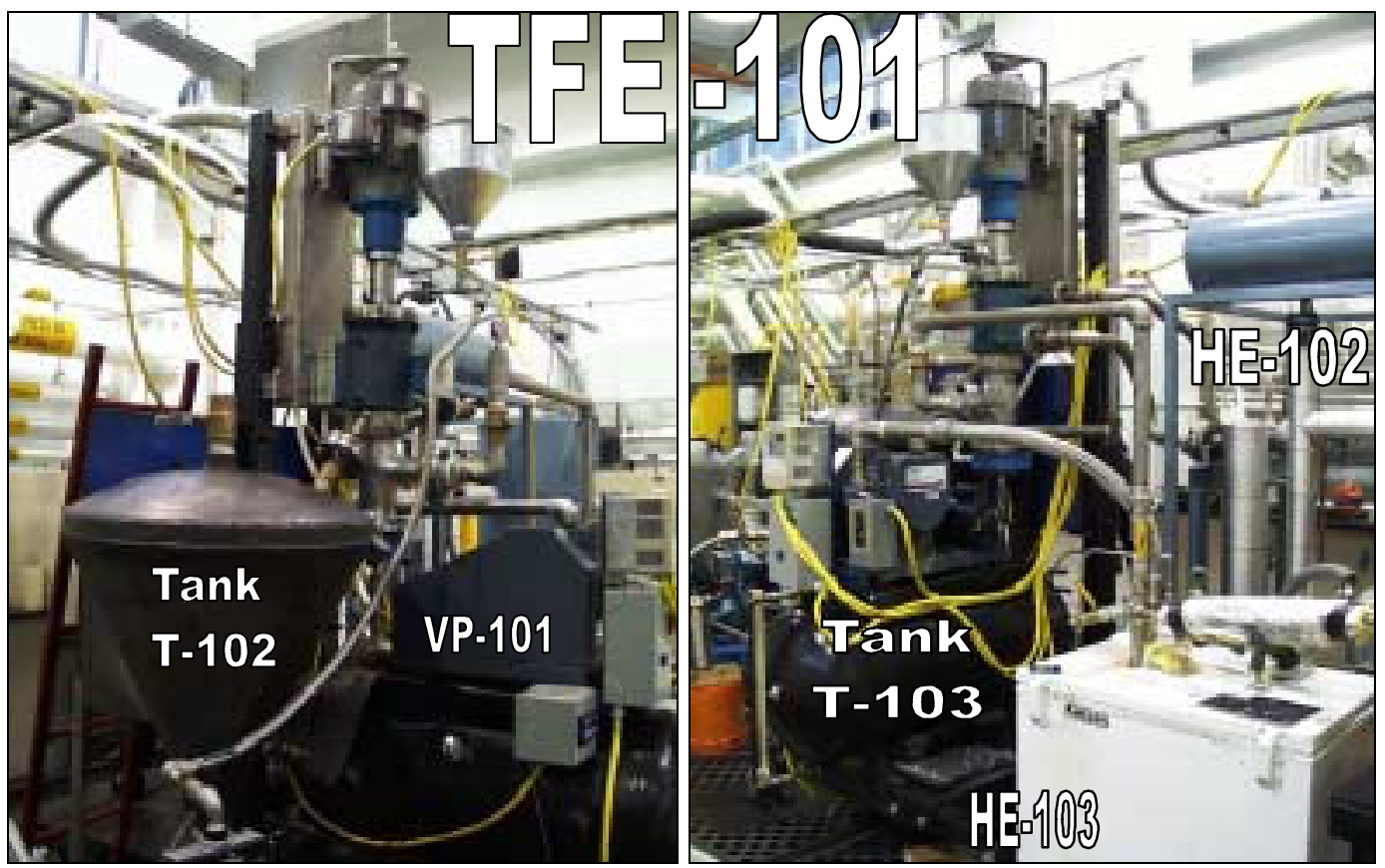

Figures 4-4 \& 4-5 Pfaudler $1.2 \mathrm{ft}^{2}$ Wiped Thin Film Evaporator

The filtrate solution leaving the centrifuge is a mixture of a large quantity of NMP ( $96 \%$ by weight) and a smaller quantity of dissolved coal (4\% by weight). In order to purify the dissolved coal, the volume of NMP must be significantly reduced. The drying process is started through the use of a Wiped Thin Film Evaporator (TFE-101) shown in Figures 4-4 and 4-5. The TFE is essentially a heated cylinder on to which the filtrate is sprayed and wiped thereby boiling off the solvent. The TFE removes a majority of the solvent by evaporation $(72 \pm 2 \%)$.

The TFE unit is actually six separate components working together: (1) the evaporator/wiper/separator (TFE-101), ((2) the supporting hot oil heater (HE-102), (3) the vacuum system (VP-101), (4) vapor trap (HE-103), (5) the product (thickened extract) tank (T-102), and (6) the condensed solvent collection tank (T-103).

- The TFE operation starts by pumping the liquid filtrate (NMP and dissolved coal) into the head of the evaporation unit by means of a gear pump (P-104). A distribution plate in the head of the TFE sprays the fluid onto the heated walls of the cylindrical vessel. The heating unit (HE-102) uses Marlotherm HT as the heating media 
(fluid) to transfer heat to the cylinder wall at a specified temperature. The filtrate is wiped around the cylindrical wall by four blades attached to a rotor that turns on the central axis at 120 RPM. The rotation rate of the wipers (blades) can be controlled. These blades are notched so that the less viscous fluid is lifted upward and the more viscous fluid is allowed to flow down to the exit port. The viscosity of the exiting fluid depends on the amount of NMP in the filtrate film. The NMP amount decreases as it is boiled off and condensed.

As the solvent evaporates from the heated surfaces, the wiper blades scrape the thickened residue away from the hot surface to an exit port. This exit port is affixed to a collection vessel (tank T-102) from which the sludge-like residue can be extracted and placed in the rotary evaporator (RV-101) for further drying

The rotor in the TFE not only rotates the distribution plate and wiper blades, but also a "squirrel cage" fan. The rotation of the fan draws the vaporized solvent from the boiling film surface to the center of the cylinder where a "cold finger" condenser condenses the solvent. An external water supply is used as the cooling source to chill the cold finger condenser. The solvent is condensed on the condenser and gravity fed into a containment vessel (Tank T-103) below the TFE where it will be recycled to the reactor. A large portion of the solvent is evaporated in the TFE ( $72 \%$ of the solvent in the filtrate fed is boiled off), and the concentration of the dissolved coal in the film is increased from 3.7 to $12 \%$ by weight

There are 5 operating conditions that control the TFE operation:

1) The flow rate of filtrate to the TFE $\left(F_{i n}\right)$.

2) The temperature of the filtrate feed to the TFE $\left(T_{\text {in }}\right)$.

3) The temperature of the heating fluid from the supporting heat exchanger $\left(T_{m}\right)$.

4) The film thickness on the wall—controlled by the blades rotation rate (RPM).

5) The internal pressure controlling the evaporation temperature $\left(\mathrm{P}^{\mathrm{sat}}\right)$.

TFE Operational parameters were initially approximated using pure water as a test fluid. Subsequent experimental testing led to the following optimal operating conditions. The fluid viscosity set the rotation rate of the blades at $350 \pm 20$ RPM. It was found that the most favorable heating fluid temperature $\left(\mathrm{T}_{\mathrm{m}}\right)$ is $208^{\circ} \mathrm{C}$ with a filtrate flow rate $\left(\mathrm{F}_{\mathrm{in}}\right)$ of 
$0.5^{\mathrm{L}} / \mathrm{min}$ and an input temperature $\left(\mathrm{T}_{\mathrm{in}}\right)$ of $100+{ }^{\circ} \mathrm{C}$. The Welch vacuum pump is sufficient to evacuate the 60-gallon vessel and all parts of the TFE to the desired operating pressure $\left(\mathrm{P}^{\text {sat }}\right)$ of below $10 \mathrm{~mm} \mathrm{Hg}$ pressure. Approximately $70 \%$ of the original volume of NMP is evaporated at these operating parameters. This seemed to be the desired amount. If too much NMP is evaporated from the coal extract, the TFE will gum up. It is noted that this will not happen with hydrogenated coal extract, which has a softening point below the boiling point of NMP. It is thought that all of the NMP can be evaporated from hydrogenated coal extract using the TFE.

Once the NMP concentration in the filtrate has been reduced, the distilled clean NMP that is collected below the TFE is pumped back into the measuring vessel (T-104). This permits a determination of the efficiency of the TFE and also permits an approximation of the quantity of NMP that remains with the coal. The thickened pitch (Figures 4-6 and 4-7) is extracted from the pitch-receiving vessel and weighed. This also helps to approximate the amount of NMP remaining with the thickened extract.

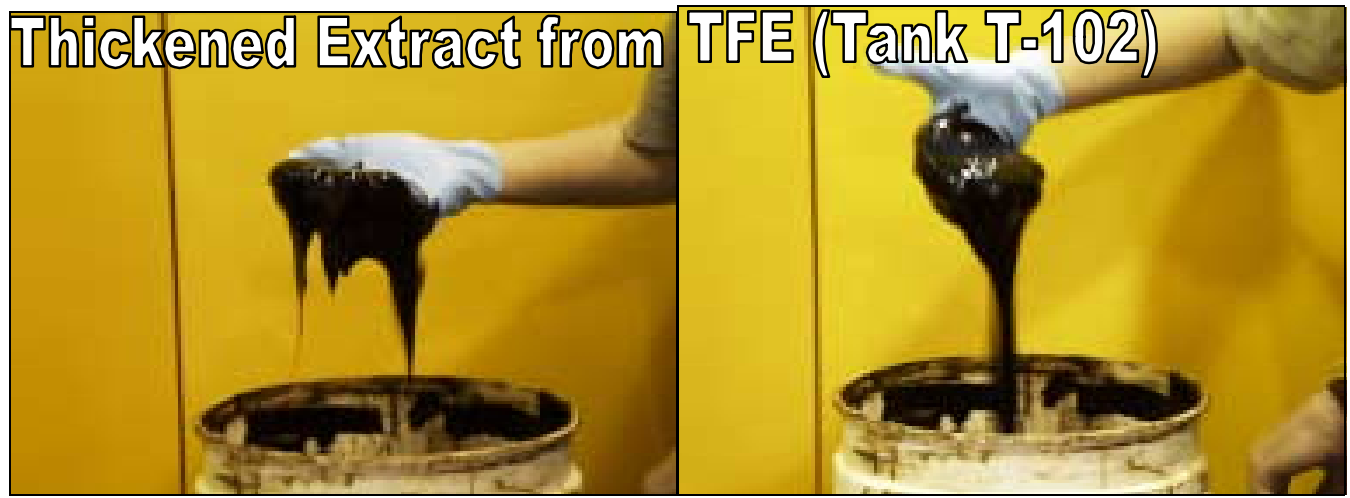

\section{Figures 4-6\&7 $\quad$ Thickened Extract Leaving Wiped Thin Film Evaporator}

\subsubsection{TFE Supporting Heat Exchanger (HE-102)}

This $20 \mathrm{~kW}$ heat exchanger was purchased as a self-standing skid unit. Heat Exchanger HE-102 uses Marlotherm HT as the heat transfer oil with an upper operating temperature limit of $320^{\circ} \mathrm{C}$. Heat exchanger $\mathrm{HE}-102$ heats the oil to the desired operating temperature of the TFE. The hot oil is then circulated over the external surface of the TFE cylinder wall supplying the heat needed to evaporate the NMP. The unit was sized properly and was equipped with a sensitive controller so the oil temperature can be kept essentially constant. 


\subsubsection{Vacuum Pump for TFE System (VP-101)}

The entire TFE system is under vacuum and the pressure is externally controlled by vacuum pump VP-101. The system pressure controls the temperature at which the solvent evaporates.

\subsubsection{Vapor Trap Heat Exchanger (HE-103)}

Located in line between the TFE and VP-101 is a cold trap/condenser (HE-103). This is a stainless steel trap of about 18 liters in volume. It is suspended in a bath of antifreeze kept in a small freezer unit. The freezer keeps the antifreeze at about $-15^{\circ} \mathrm{C}$. This prevents any of the NMP from migrating to the vacuum pump. The pump oil is changed at regular intervals to ensure limited contamination and continued proper operation.

\subsubsection{Product or "Thickened Extract" Tank (T-102)}

Tank T-102 shown in Figure 4-4 is a 35-gallon cone shaped tank. This tank is cone shaped to facilitate the removal of the thickened extract. This tank was an addition designed after the first few runs with coal. The product (thickened extract) from the TFE has the consistency of a tar as shown by Figures 4-6 and 4-7. This tank is designed so that the product could easily flow out the bottom 3" port into the cans designed for the Rotovap. This tank is also equipped with high-pressure air connection to forcefully remove the product in case the TFE was not run properly, and the extract is too thick.

\subsubsection{Condensed Solvent (NMP) Collection (T-103)}

The solvent collection vessel (Tank T-103) contains pure NMP and is equipped with a pump (P-103) that transports the NMP back to the CSTR for reuse. This tank is a 60-gallon air tank mounted in the horizontal position under the TFE to collect the gravity fed condensed solvent. This tank is also fitted with a sight glass to monitor the rate at which the solvent is evaporated. 


\subsubsection{Rotary Evaporator (RV-101)}
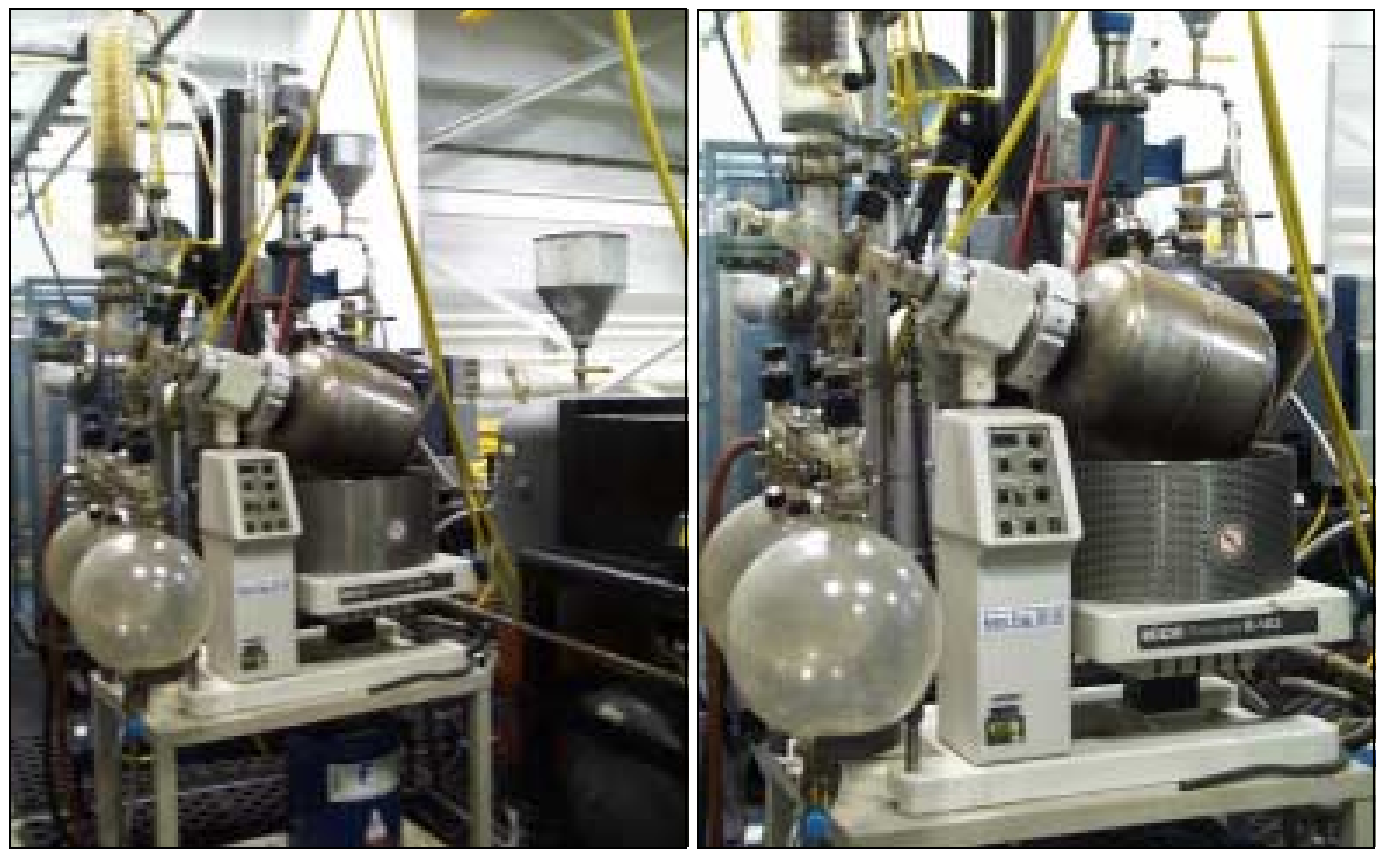

\section{Figures 4-8 and 4-9 Modified Buchi R-135 Rotary Evaporator with Steel Tank}

The next step in the process is to further remove the NMP from the thickened extract product coming from the TFE. This is done using a rotary evaporation unit (Rotovap) shown in Figures 4-8 and 4-9. The Rotovap unit (RV-101) is essentially a thin film evaporator. This system is composed of three components: (1) the Rotary Evaporator or Rotovap (RV-101) that processes the thickened extract from the TFE, (2) supporting vacuum system (VP-102), and (3) vapor trap (HE-105).

The Rotovap takes the thickened extract from the TFE and dries it into a semisolid containing about $50 \%$ by weight-reconstituted coal. This unit was the only piece of equipment that was utilized both in the CEPU and in the laboratory process. However, some modification was done on the Rotovap enabling it to handle the higher rate of processing. This modification was done on the rotating vessel that holds the material being processed. The rotating vessel currently used is a modified steel Freon bottle instead of the previous glass bulb. The steel bottle has a larger volume than the standard 20-liter glass round bottom bulb. Further, the steel bottle cannot be broken as easily. 
The Rotovap is a batch unit that uses a hot oil bath to evaporate the NMP. The process is started by placing the tar like thickened extract from the TFE into a steel bulb. The steel bottle is partially filled with the thickened extract. About 3 gallons of material constitutes a batch. The steel bottle is attached to the Rotovap by a rotating flange. Once attached to the Rotovap, the steel bottle is lowered into a hot oil bath and rotated. The system is then evacuated to below $10 \mathrm{mmHg}$ using vacuum pump P-102.

As the steel bottle is rotated, the thickened extract forms a thin film over the internal surface of the bottle. The hot oil provides heat to the film from which the NMP is evaporated. The NMP vapors are then drawn by the Welch vacuum pump (VP-102) into the condenser. The condenser is chilled using cold water. The NMP vapors are condensed to a liquid and collected in glass bulbs for reuse.

As the solvent is removed from the thickened extract, the solid coalesces into large agglomerates. These have the appearance of small footballs or a large softball. This unbalances the rotating vessel and the process is discontinued. This material is then removed, ground, and placed in trays and put in the drying ovens for the final drying.

The Rotovap and the vacuum drying ovens share the same vacuum system. This system is composed of the vacuum pump (VP-102), and supporting vapor trap (HE-105). These are both explained further in sections 4.5.1 and 4.5.2 respectively. 


\subsubsection{Vacuum Drying Ovens:}

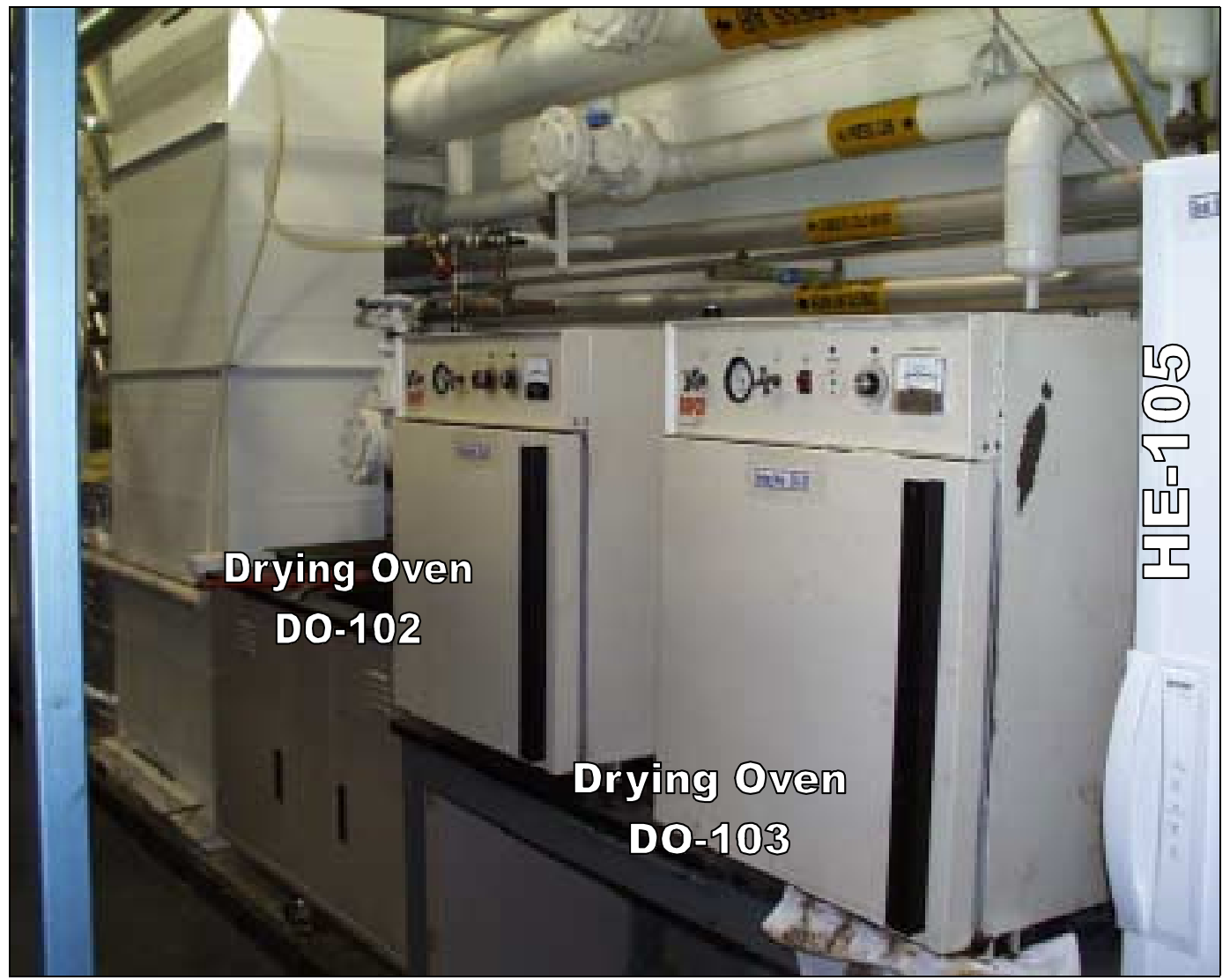

\section{Figure 4-10 Processed Coal and Pitch Drying Ovens and Heat Exchanger}

The vacuum drying ovens evaporate the NMP contained in the product from the rotovap and the undissolved coal stream from the centrifuge. The Rotovap process removes the solvent from the reconstituted coal to less than $50 \%$ by weight. The remaining solvent can be removed to less than $2 \%$ by vacuum drying. The undissolved processed coal leaving the centrifuge contains $55 \pm 5 \%$ NMP. It is essential that this NMP also be recovered by vacuum drying.

In the vacuum drying operation, the semi-dried, thickened extract is ground and spread out in stainless steel trays. The purpose of the trays and grinding is to expose as much surface as possible. The undissolved coal is spread out in the trays and the trays are then placed in the vacuum drying ovens shown in Figure 4-10. Each oven holds three trays and each tray holds about $3 \mathrm{~kg}$ of semi-dry, thickened extract or undissolved coal. 
The ovens are heated to $205^{\circ} \mathrm{C}$ under a $10 \mathrm{~mm} \mathrm{Hg}$ vacuum. The NMP vapors given off are drawn through condenser HE-105 by vacuum pump VP-102. This condenser is chilled to $-13^{\circ} \mathrm{C}$ by circulating antifreeze from the 45 -gallon sump (reservoir) located below tank T-106 as shown in Figure 4-11. The NMP condensate is collected in tank T106 and recycled for re-use. A small nitrogen flow is permitted to pass through the ovens to help draw the NMP vapors to the condenser (HE-105).

As the semi-dry granular extract is dried, it is stirred so that more surface area is exposed. This process is repeated until no more condensate is detected at the condenser. At this time it is assumed that the coal extract is free of solvent. Ultimate analysis of the solid reconstituted pitch showed the extract contained less than $2 \% \mathrm{NMP}$, assuming that all solvent retention is in the extract. This indicated that indeed most the solvent (greater than $99.9 \%$ ) has been removed and recycled or re-use.

\subsubsection{Vacuum Pump for Rotovap \& Vacuum Drying Ovens (VP-102)}

The vacuum pump VP-102 is used to lower the pressure of the Rotovap and the drying ovens. This vacuum pump is identical to VP-101.

The entire Rotovap and drying oven systems are maintained under vacuum and the pressure is externally controlled by vacuum pump VP-102. This vacuum pump draws the NMP vapors given off from the semi-dry granular extract in the drying ovens through the condenser HE-105 where the NMP is condensed and recycled.

\subsubsection{Vapor Trap/Condenser Heat Exchanger (HE-105)}

Located in line between the drying ovens and VP-102 is a cold trap/condenser (HE-105). This heat exchanger is a cast iron and copper two-pass U-tube type heat exchanger. This heat exchanger serves as the condenser for the drying ovens and vapor trap for the vacuum pump.

The freezer unit shown in Figure 4-11 keeps the heat exchanger and a 45-gallon antifreeze sump (reservoir) chilled to about $-13^{\circ} \mathrm{C}$. The antifreeze is circulated from the reservoir, through the tube side of the condenser, and back top the reservoir by an air operated diaphragm pump. This cooling media condenses the NMP vapors on the shell 
side of the heat exchanger. This low temperature cooling media prevents any of the NMP vapors from migrating to the pump. However, the pump oil is changed at regular intervals to ensure limited contamination and continued proper operation.

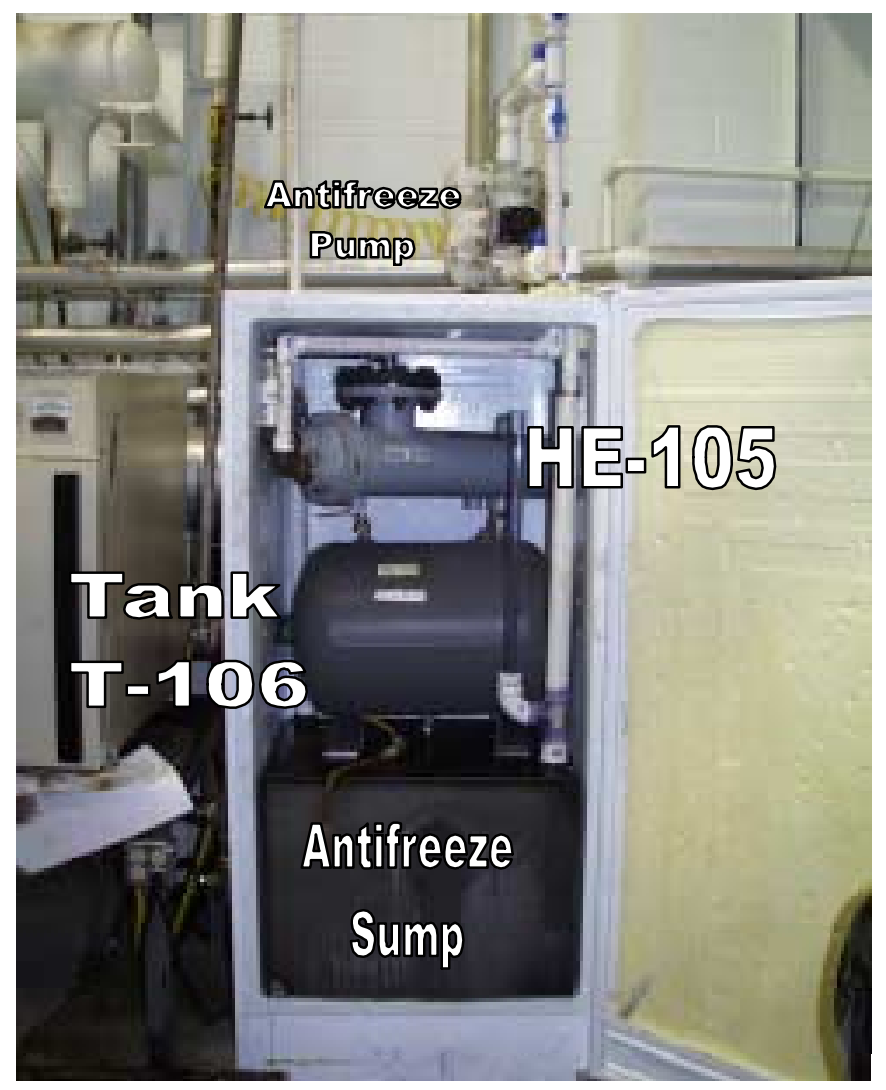

Figure 4-11 Drying Oven Condenser (HE-105) $\&$ Collection Tank (T-106) 


\section{CHAPTER 5}

\section{MATERIAL BALANCE}

One of the major goals of this research project was to perform a material balance over the CEPU to determine the actual extract yield, solvent loss, and percent mass balance closure (\% MBC) on the weight of raw coal, extract and residue. The amount of solvent loss is a major concern. Loosing a small percentage of the original solvent would greatly affect the cost of the coal-based carbon pitch produced. Under normal operation conditions the solvent can leave the system in only three ways: as residual solvent in the dried pitch, as solvent not recovered in the dried processed coal waste (residue), and solvent loss cleaning up the solvent/water mixture taken off of the reactor by condenser (HE-101) as explained in section 4.1.0.

Using the ultimate analysis preformed on the coal, centrifuged processed coal, dried centrifuged processed coal, and the dried extract or "pitch", a material balance on the solvent can be done over the entire system. The overall solvent material balance for this research neglected the solvent that might be lost in cleaning up the solvent/water mixture from the reactor's condenser (HE-101). The amount of solvent recovered from the solvent/water mixture would be a function of how much water the coal contained, whether or not the coal was dried, and what type of clean up method was used.

The research presented here focused on the amount of residual or solvent retention in the extract and residue. Currently there is no quantitative measurement of the solvent retention, however, an apparent solvent retention level can be calculated using the ultimate analysis of the raw coal, extract and residue. The major assumption in this calculation is that all increased nitrogen in the extract and residue originates from the nitrogen in NMP. It may however, originate from the nitrogen in the air or the nitrogen that is used to purge the extraction vessel and the vacuum ovens (Gerstner 1989). Also, the absolute amount of nitrogen in the raw coal, extract, and residue is small. This smaller number can lead to large errors in the determination of the amount of increased nitrogen. Therefore, the results are considered to be an inflated value for the level of solvent retention in the extract and can be assumed to be the worst-case scenario. 


\subsubsection{Overall Material Balance}

A few terms need to be defined to calculate the overall material balance for the coal and solvent over the CEPU.

First, the $\%$ yield, or percent extraction is the percentage of the feed coal that is dissolved or extracted. The \% yield is calculated using the Equation 5-1 below.

$$
\% \text { yield }=\frac{(\text { wt. of raw coal }- \text { wt. or residue })}{\text { wt. of raw coal }} * 100
$$

This is the most practical method, in determining how well the coal was extracted by the NMP. However, it is not the most accurate due to the fact that it considers the ash or moisture content of the raw coal that are not extractable.

The Moisture and Ash Free percent yield calculation (\% yield MAF) does not take into account the portion of the raw coal that is not extractable material, namely the water and ash. So the amount of water and ash is subtracted out of the weight of the coal to determine the amount of organic material that is extractable. This is a more accurate and consistent basis of which to compare the performance of various coals in extraction procedure (Gerstner 1989). For this calculation, the extract is assumed to be essentially dry and ash-free which is confirmed by the proximate analysis. The \% yield (MAF) is calculated using Equation 5-2 shown below.

$$
\% \text { yield }(\mathrm{MAF})=\frac{\mathrm{wt} . \text { of extract }}{(1-\mathrm{A} \%-\mathrm{M} \%) * \text { wt. of raw coal }} * 100
$$

$$
\begin{aligned}
& \text { where: } \mathrm{A}=\text { Ash from proximate analysis of raw coal } \\
& \mathrm{M}=\text { Moisture from proximate analysis of raw coal }
\end{aligned}
$$

Percent Mass Balance Closure (\% MBC) is calculated using Equation 5-3. This is an indication of the operational performance of the CEPU by weight o raw coal. A \% MBC greater that $2 \%$ indicates that there is significant solvent retention. A \% MBC less that $-2 \%$ indicates that there is a problem somewhere in the system. 


$$
\% \mathrm{MBC}=\frac{(\text { wt. of extract }+ \text { wt. of residue })-\text { wt. of raw coal }}{\text { wt. of raw coal }} * 100
$$

The apparent percent nitrogen retention is back calculated by determining the increase in nitrogen content of the extract and the residue as compared to the raw coal.

$$
\begin{gathered}
\text { increased } \mathrm{N}=\left(\frac{\% \mathrm{~N}_{\mathrm{R}}}{100} * \text { wt. of residue }+\frac{\% \mathrm{~N}_{\mathrm{E}}}{100} * \text { wt. of extract }\right)- \\
\frac{\% \mathrm{~N}_{\mathrm{Raw}}}{100} * \text { wt. of raw coal }
\end{gathered}
$$

$$
\text { where: } \begin{array}{ll} 
& \mathrm{N}_{\mathrm{R}}=\% \text { Nitrogen of Residue }{ }^{\mathrm{a}} \\
& \mathrm{N}_{\mathrm{E}}=\% \text { Nitrogen of Extract }{ }^{\mathrm{a}} \\
& \mathrm{N}_{\mathrm{Raw}}=\% \text { Nitrogen of Raw Coal }{ }^{\mathrm{a}} \\
& { }^{\mathrm{a}} \text { From Ultimate Analysis Wet Basis }
\end{array}
$$

The apparent level of solvent retention can then be calculated by relating the increased nitrogen content to the mass ratio of nitrogen in NMP in the following manner:

$$
\% \text { Solvent Retention }=\frac{\text { increased } \mathrm{N} * \frac{\mathrm{MW}_{\mathrm{NMP}}}{\mathrm{MW}_{\mathrm{N}}}}{\text { wt. or raw coal }} * 100
$$

where: $\quad \mathrm{MW}_{\mathrm{NMP}}=99.1$

$$
\mathrm{MW}_{\mathrm{N}}=14
$$

Using the Ultimate Analysis in Appendix B, an overall material balance can be done over the system. The results for the material balance are shown in Table 5-1. 


\subsubsection{Material Balance Over Individual Units}

Now that the overall material balance for the system had been considered, the material balances over the five main components of the system as seen on the Block Flow Diagram in Figure 3-2 will be considered.

- Continuous Stirred Tank Reactor (CSTR)

(R-101)

- Centrifuge

- Wiped Thin Film Evaporator (TFE)

(TFE-101)

- Rotary Evaporator (Rotovap)

(RV-101)

- Drying Ovens

(DO-101-3)

This material balance is important for calculating the energy balance over the system next.

\subsubsection{Material Balance Over Continuous Stirred Tank Reactor (CSTR)}

(R-101)

Again the CSTR (reactor) is loaded with a 10:1 ratio by weight of NMP to coal. It is heated to $202^{\circ} \mathrm{C}$ while stirring. The reactor is then kept at $202^{\circ} \mathrm{C}$ for one hour. NMP dissolves the Upper Powleton coal giving the yield of $36 \pm 2 \%$ and a yield (MAF) of $39 \pm 2 \%$ shown for the overall material balance. The slurry of dissolved coal in NMP and undissolved coal is then fed to the Centrifuge (Ct-101).

The fresh coal used in the CEPU is not dried prior to processing. Therefore the reactor is designed so that the water can be driven off, condensed, and cleaned up. Again, the overall material balance for this research neglected the solvent that might be lost in cleaning up the solvent/water mixture from the reactor's condenser (HE-101). The amount of solvent recovered from this would be a function of how much water the coal contained, whether or not the coal was dried, and what type of clean up method was used.

The average amount of solvent driven of by the reactor appeared to be 1 to 2 liters. This volume of solvent loss was not thought to be enough to affect the $\%$ yield. The condensed NMP is collected until there was enough to load the reactor with an appreciable quantity. The reactor then serves as a distillation column by heating several batch loads to the boiling point $\left(202^{\circ} \mathrm{C}\right)$ of NMP. Once at $202^{\circ} \mathrm{C}$ the essentially pure NMP solution is cooled and pumped into the upright tank (T-104) to be used in the next run. The water and NMP vapors that are driven off are condensed and collected. The reactor is always operated at atmospheric pressure. Therefore, using the glass collection flask affixed to the bottom of the condenser (HE-101), the boiling temperature is noted. 
This boiling temperature is used to calculate the amount of water in mixture using the vapor/liquid equilibrium diagram shown in Figure B-4 of Appendix B. The procedure listed above is repeated until the mixture contains only 40-mole \% NMP.

\subsubsection{Material Balance Over Centrifuge}

(Ct-101)

Again, the centrifuge is fed the slurry of dissolved coal in NMP and undissolved coal from the reactor by pump P-101 at 2 liters $/ \mathrm{min}$. The feed is approximately $92 \%$ NMP $3 \%$ Dissolved coal (extract), and $5 \%$ undissolved coal by weight. The centrifuge, operating at $2200 \mathrm{~g}$, separates the solution of dissolved coal and NMP from the solid undissolved coal.

The dissolved coal in NMP leaves the centrifuge and is feed to the TFE where a major portion of the NMP is then driven off. The TFE feed stream contains approximately 96.3 weight $\%$ NMP and 3.7 weight $\%$ extract.

The undissolved coal leaves as a moist granular solid containing $55 \pm 2$ weight $\%$ NMP. This solid resembles damp potting soil. NMP is still entrapped in the undissolved coal particles, yet the particles are not saturated. The undissolved coal is then placed in a vacuum drying oven where the NMP is driven off.

\subsubsection{Material Balance Over Wiped Thin Film Evaporator (TFE) (TFE-101)}

The TFE is utilized to drive off a major proportion of the NMP from the extract. The TFE drives off $72 \pm 2 \%$ of the NMP in the feed stream from the centrifuge using the $20 \mathrm{~kW}$ supporting heat exchanger. The evaporated NMP off is condensed and recycled for another run. The product of the TFE is a "thickened extract". This thickened extract, containing $89 \%$ NMP by weight and $11 \%$ extract, is then feed to the Rotary Evaporator (Rotovap).

\subsubsection{Material Balance Over Rotary Evaporator (Rotovap)}

(RV-101)

The thickened extract from the TFE is fed to the Rotovap in batch loads. The Rotovap uses a hot oil bath to drive off approximately $88 \%$ by weight of the NMP contained in the thickened extract. This is $24 \%$ by weight of the NMP in the filtrate from the centrifuge. The product leaving the rotovap is a semi-solid mass of $50 / 50 \%$ by 
weight extract/NMP. This solid is sent to a vacuum drying oven to complete the drying process.

Notice that the Rotovap drives off $1 / 3$ the amount of NMP in the filtrate as that evaporated in the TFE. Therefore, the addition of the TFE reduces the number of batches in the Rotovap by a factor 4 .

\subsubsection{Material Balance Over Drying Ovens}

(DO-101-3)

The Drying Ovens are the final step in the process. The solid extract leaving the Rotovap and the residue leaving the centrifuge are sent to the Drying Ovens to remove the NMP down to less than $2 \mathrm{wt} \%$. Note that this is a calculated number from the Ultimate Analysis of the fresh coal, dried waste coal, and final dried coal-based carbon pitch.

\section{Table 5-1 Material Balance Results}

\begin{tabular}{llll} 
Coal & $36 \%$ Extract \\
& $64 \%$ Residue \\
& $36 \pm 2 \%$ & \\
Yield & $39 \pm 2 \%$ & \\
Yield (MAF) & $<1 \%$ & \\
MBC & \multicolumn{3}{l}{} \\
Solvent Retention & $<0.7 \%$ (by wt of Raw Coal) \\
& $<2 \%$ (by wt of Extract) \\
& \multicolumn{3}{l}{} \\
& Fresh Coal & Extract & Residue \\
& $100 \%$ & $36 \%$ & $64 \%$ \\
\% Volatile Matter & 26 & 30 & \\
\% Fixed Carbon & 64 & 68 & 13200 \\
BTU/lb & 14200 & 15000 & 77 \\
\% Carbon & 84 & 87 & 2.7 \\
\% Hydrogen & 3.4 & 3.8 & 0.47 \\
\% Nitrogen & 0.6 & 1.2 & 3.0 \\
\% Oxygen & 4.1 & 5.2 & 0.7 \\
\% Sulfur & 0.8 & 0.7 & $8.5 \%$ \\
\% Ash & $6.8 \%$ & $<0.6 \%$ &
\end{tabular}




\section{Coal \\ (70 mesh) \\ CEPU Block Flow Diagram}

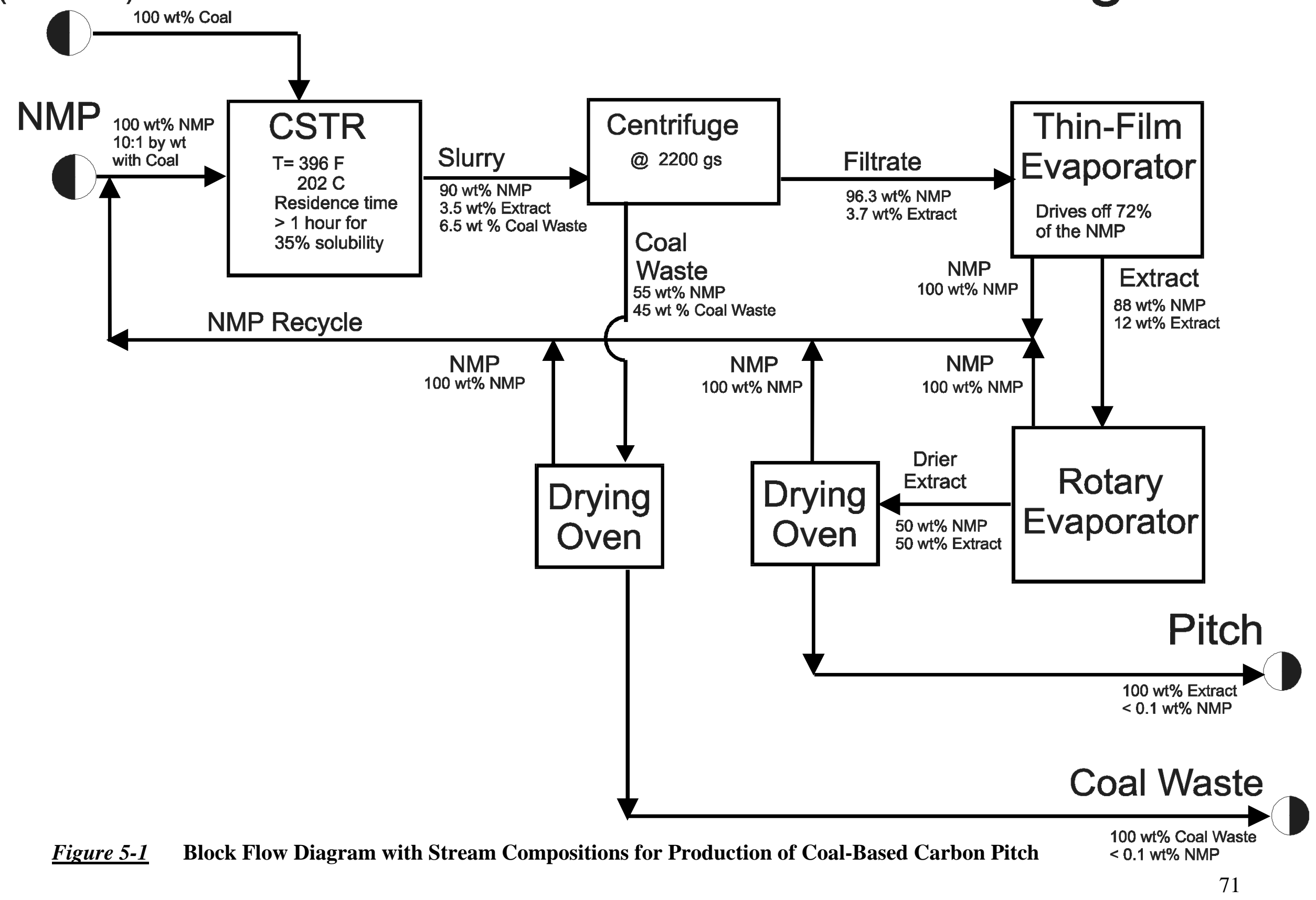




\section{CHAPTER 6}

\section{ENERGY BALANCE}

An energy balance over the CEPU can be calculated once a detailed material balance for the CEPU is complete. The energy balance for this system focuses on the amount of thermal energy associated with the operation of four of the five main components and their supporting units (i.e. heat exchangers) of the CEPU. The Centrifuge is not mentioned in this section due to the fact that there is only mechanical work done by the centrifuge, no thermal. Thus the four main components are:

- Continuous Stirred Tank Reactor (CSTR)

- Wiped Thin Film Evaporator (TFE)

- Rotary Evaporator (Rotovap)

$(\mathrm{RV}-101)$

- Drying Ovens

(DO-101-3)

\subsubsection{Thermal Properties of NMP}

The physical properties of NMP and coal are needed to calculate the energy balance. Most of NMP's physical properties can be found in Appendix B. Listed below are the major thermal properties of NMP needed for the energy balance.

$$
\begin{array}{lc}
\text { Specific Heat }\left(\mathrm{Cp} \mathrm{pMP}_{\mathrm{NM}}\right): & \begin{array}{c}
1.67 \mathrm{~J} / \mathrm{kg} /{ }^{\circ} \mathrm{C} \text { at } 20^{\circ} \mathrm{C} \\
\mathrm{C} \mathrm{p}_{\mathrm{NMP}}=3.37 * 10^{\wedge}-3 *(\mathrm{~T})+1.59 \\
\left(\mathrm{Cp}\left[\mathrm{kJ} / \mathrm{kg}{ }^{\circ} \mathrm{C}\right], \mathrm{T}\left[{ }^{\circ} \mathrm{C}\right]\right)
\end{array} \\
& \begin{array}{c}
\mathrm{k}_{\mathrm{NMP}}=-1^{*} 10^{\wedge}-4(\mathrm{~T})+0.1954 \\
\left(\mathrm{~T}\left[{ }^{\circ} \mathrm{C}\right], \mathrm{k}_{\mathrm{NMP}}\left[\mathrm{W} / \mathrm{M}{ }^{\circ} \mathrm{C}\right]\right)
\end{array} \\
\text { Thermal Conductivity }\left(\mathrm{k}_{\mathrm{NMP}}\right): & 53 \mathrm{~kJ} / \mathrm{kg} \text { at } 20^{\circ} \mathrm{C}
\end{array}
$$

Even though NMP makes up the majority of the composition of the streams in the CEPU, its concentration drops as a batch progresses through the CEPU process. Therefore, some of the thermal properties of coal need to be defined.

\subsubsection{Thermal Properties of Coal}

The specific heat of coal is a thermal property needed to calculate the energy associated with heating and cooling the coal in the CEPU. As seen in Figure 6-1, the 
specific heat of coal increases with volatile matter and temperature (Volborth 1987). Figure 6-1 shows the specific heat of coal with $25 \%$ volatile matter at $200^{\circ} \mathrm{C}$ to be about $1.6 \mathrm{~kJ} / \mathrm{kg} \mathrm{K}$. The coal used in this research project contains less than $25 \%$ volatile matter. As a result, the research presented here assumes the energy need in heating and cooling of coal to be the same as that of NMP. NMP has a specific heat of $1.67 \mathrm{~J} / \mathrm{kg} /{ }^{\circ} \mathrm{C}$ at $20^{\circ} \mathrm{C}$ and increase with temperature. This is greater than that of coal at $202^{\circ} \mathrm{C}$ (the maximum operating temperature of the CEPU).

The assumption that coal has the same specific heat as NMP may lead to slightly inflated values for the energy balance. However, the numbers calculated here are theoretical values and do not take into account the equipment operating efficiency. Therefore, the inflated values are thought to be an order of magnitude less that the adjusted values for equipment efficiency. If one is so inclined, more detailed models for the specific heats of coals are presented by Kirov (1960) and van Krevelen (1961).

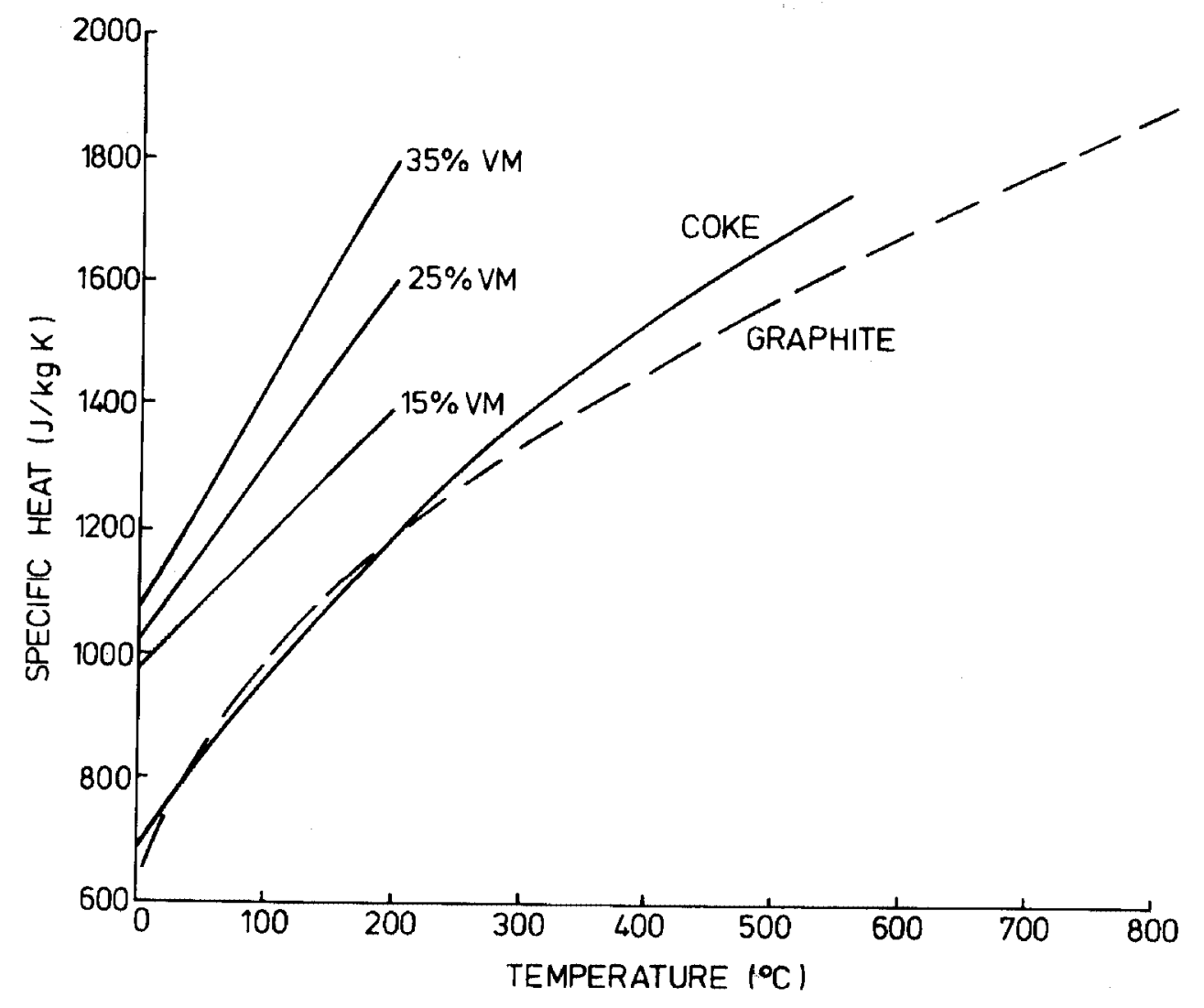

Figure 6-1 Typical Values of the Specific Heats of Coal, Coke and Graphite. (Volborth 1987) 
The thermal conductivity of coal is the second property that is important for the energy balance on the Rotovap and drying ovens. The thermal conductivity of coal is calculated using Equation 6-3 (Volborth 1987).

$$
\frac{1}{\mathrm{k}_{\text {Coal }}}=\left(\frac{\mathrm{C}}{1.47}+\frac{\mathrm{H}}{.0118}\right) *\left[\left(\frac{273}{\overline{\mathrm{T}}}\right)^{\wedge} 0.5\right]
$$

Where: $\mathrm{C}$ and $\mathrm{H}$ are mass fractions of Carbon and Hydrogen, and $\mathrm{T}$ is absolute temperature in $\mathrm{K}$.

At $0^{\circ} \mathrm{C}(\mathrm{T}=273 \mathrm{~K})$, the equation predicts the measured thermal conductivity of amorphous carbon $(1.47 \mathrm{~W} / \mathrm{m} \mathrm{K})$ for the limiting case of pure carbon $(\mathrm{C}=1)$. For a typical bituminous coal $(\mathrm{C}=0.85, \mathrm{H}=0.05)$ at $0^{\circ} \mathrm{C}$, the equation predicts a thermal conductivity of $0.21 \mathrm{~W} / \mathrm{m} \mathrm{K}$. This value lies within the range of literature data for monolithic specimens of bituminous coal (Volborth 1987).

\subsubsection{Energy Balance on The Continuous Stirred Tank Reactor (CSTR) (R-101)}

Again, the CSTR (reactor) is used to heat the fresh coal/NMP solution from $25^{\circ} \mathrm{C}$ to $202^{\circ} \mathrm{C}$. Loaded with $100 \mathrm{~kg} \mathrm{NMP}$ and $10 \mathrm{~kg}$ coal, this temperature increase of $177^{\circ} \mathrm{C}$ requires $38.4-\mathrm{MJ}$ of thermal energy assuming the average specific heat of $1.97-\mathrm{kJ} / \mathrm{kg}{ }^{\circ} \mathrm{C}$ from Equation 6-1. Assuming that a max of 2 liters of NMP and water are driven off and condensed by condenser (HE-101), the extra energy needed is 1.1 MJ. This brings the total to $39.5 \mathrm{MJ}$ of energy needed to heat a batch.

The reactor was designed with a 26 rope heaters operating each at 500W, generating a total of $13 \mathrm{~kW}$. Experimental results have shown that it takes 2 hours to bring the mixture of NMP and coal to a boil. Figure 6-2 shows the heating rate for the CSTR (R-101) with $100 \mathrm{~kg}$ pure $\mathrm{H}_{2} \mathrm{O}, 100 \mathrm{~kg}$ pure NMP, and a batch of $100 \mathrm{~kg}$ NMP and $10 \mathrm{~kg}$ of coal. 


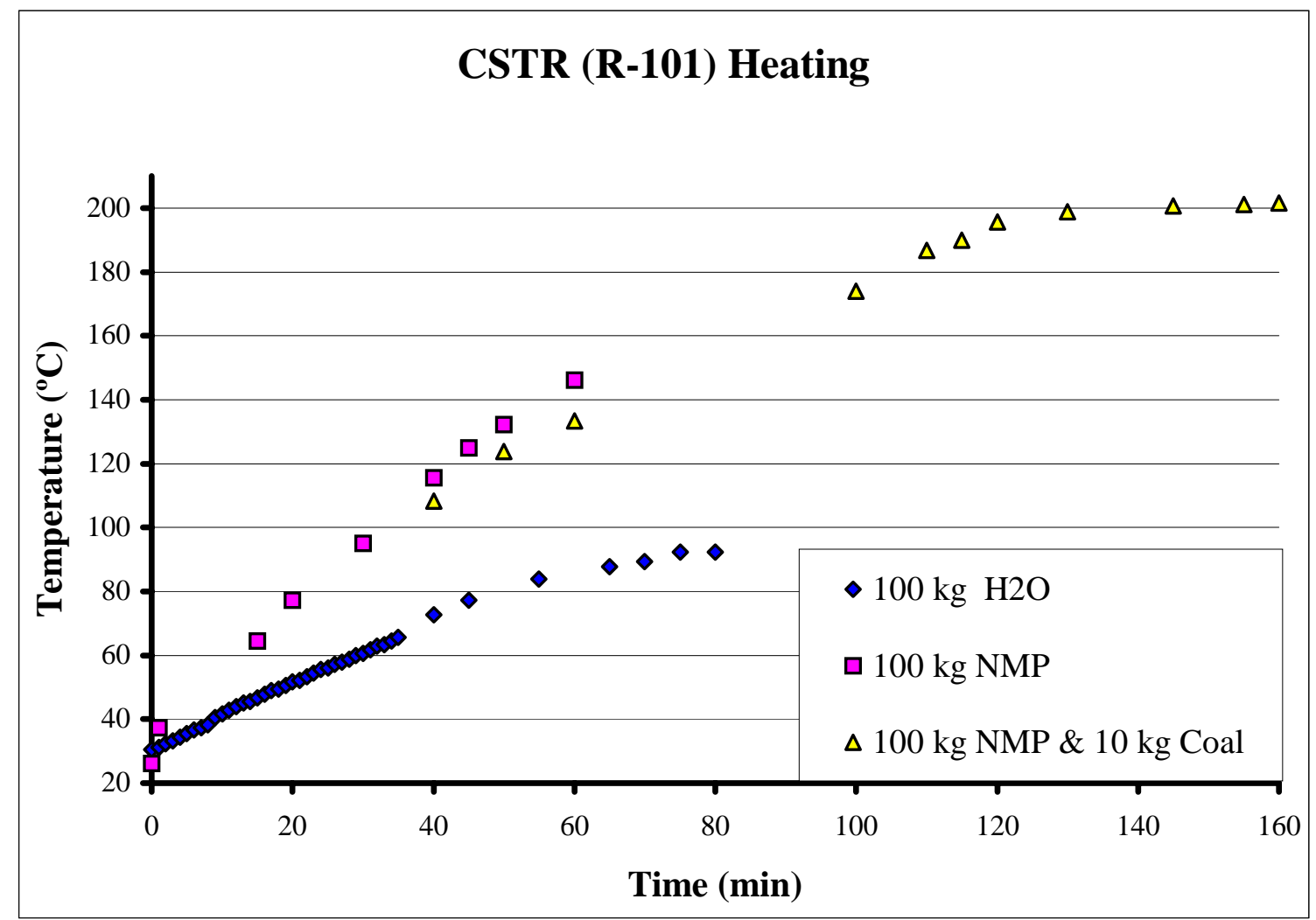

\section{Figure 6-2 CSTR (R-101) Temperature Profile for Heating}

The heaters produce a total of 93.6 MJ of energy during the two hours of heating, assuming that the heaters all work at $100 \%$. This makes the reactor's heaters about $42 \%$ efficient in heating. This low efficiency may be due to some heaters not working, heating of the metal walls and piping of the reactor itself, heat loss by convection to the surroundings, to the room temperature $\mathrm{N}_{2}$ purge, and the vapors that are condensed and allowed to flow back into the reactor.

The reactor was cooled to calculate how much heat was actually lost by the reactor. The cooling temperature profile was plotted in Figure 6-3. From this figure the rate of heat loss by the reactor can be calculated. 


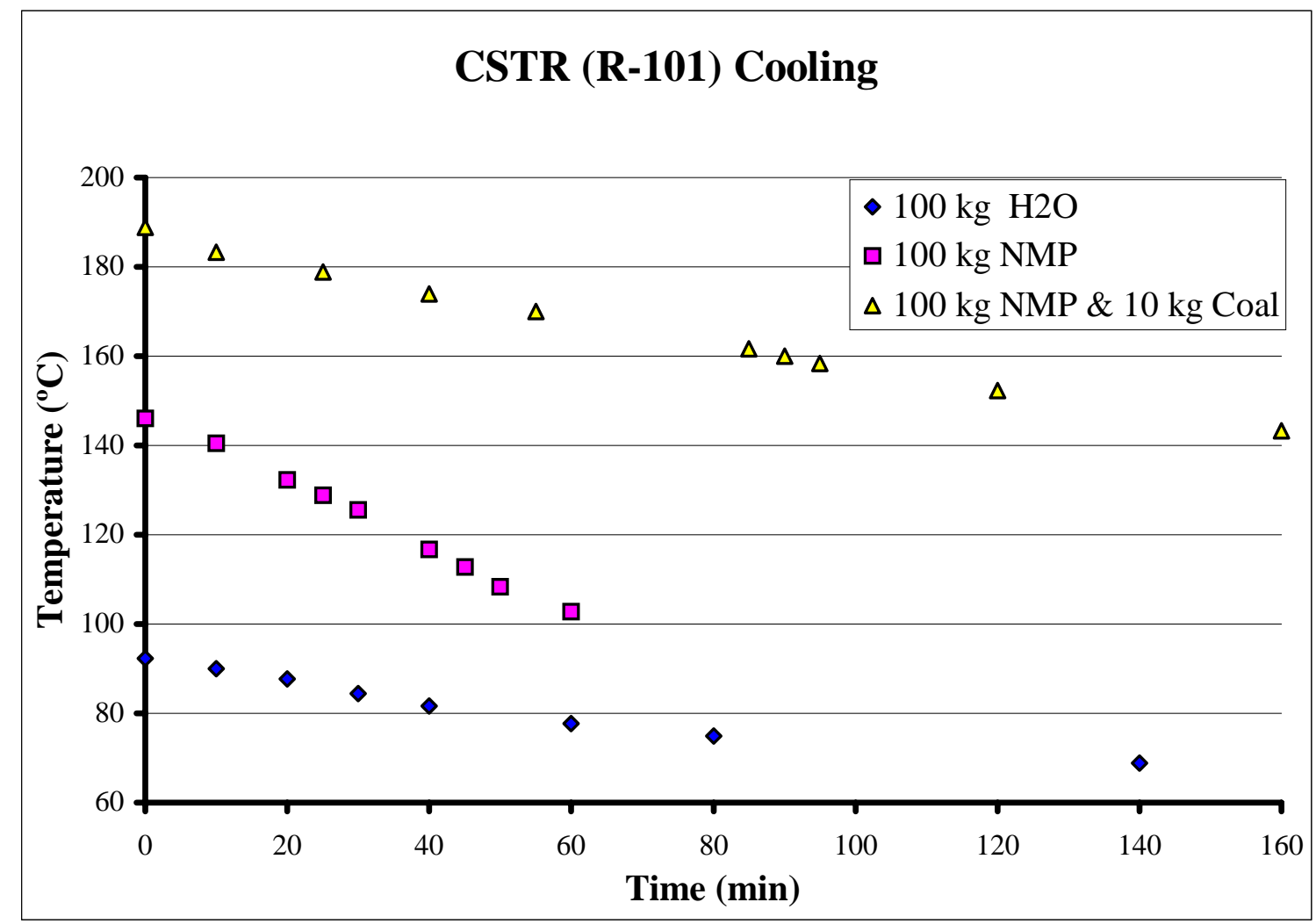

\section{Figure 6-3 CSTR (R-101) Temperature Profile for Cooling the Reactor Without Supporting Heat Exchanger HE-104}

Figure 6-3 shows the rate of heat loss of the reactor under three different conditions. Notice that the NMP solution cooled faster than the NMP/coal slurry even thought it was at a lower temperature. This is because the pure NMP solution was circulated through the un-insulated recycle pipes while it was cooling, and the NMP/coal slurry was not. The reactor cooled down $28{ }^{\circ} \mathrm{C}$ in the time of 2 hours. Assuming that only the NMP/coal mixture was cooled, a calculated rate of 3.3 MJ per hour was lost at an average rate off $0.92 \mathrm{~kW}$.

Along with the $13 \mathrm{~kW}$ rope heaters, the reactor has two supporting heat exchangers (HE-101 \& 104). These heat exchangers use tap water as the cooling media. HE-101 condenses the vapors driven off from the reactor. HE-104 cools the NMP/coal slurry in the reactor before it is pumped to the centrifuge to stay within operational limits of the unit. 


\subsubsection{Reactor $\mathrm{NMP} / \mathrm{H}_{2} \mathrm{O}$ Vapor Condenser Heat Exchanger}

(HE-101)

Heat Exchanger HE-101 uses tap water on the tube side as a cooling medium to condense NMP and water vapors boiled off of the NMP/coal slurry in the reactor on the shell side. This stainless steel heat exchanger was sized so that it could handle a thermal rate well above the maximum reactor output of $13 \mathrm{~kW}$. The reactor operating at $100 \%$ efficiency can vaporize a maximum rate $1.45 \mathrm{~kg} / \mathrm{min}$ of NMP.

The tube side of the stainless steel heat exchanger transfers heat by forced conduction of water, and the shell side of the heat transfer transfers heat by condensation of vapor. The temperate driving force from shell side to the tube side is more than $180^{\circ} \mathrm{C}$.

Using equation 6-4 (Holman 1997), the overall heat-transfer coefficient $\left(\mathrm{U}_{\mathrm{o}}\right)$ for the shell side was calculated to be $1480 \mathrm{~W} / \mathrm{m}^{2}{ }^{\circ} \mathrm{C}$. The maximum rate of heat transfer $\left(\mathrm{Q}_{\max }\right)$ for this heat exchanger was calculated to be over $400 \mathrm{~kW}$ using equation 6-5 (Holman 1997).

$$
\mathrm{U}_{\mathrm{o}}=\frac{1}{\frac{\mathrm{r}_{\mathrm{o}}}{\mathrm{r}_{\mathrm{i}}} \frac{1}{\mathrm{~h}_{\mathrm{i}}}+\frac{\mathrm{r}_{\mathrm{o}}}{3.14} * \frac{\ln \frac{\mathrm{r}_{\mathrm{o}}}{\mathrm{r}_{\mathrm{i}}}}{\mathrm{k}_{\mathrm{ss}}}+\frac{1}{\mathrm{~h}_{\mathrm{o}}}}
$$

where: $r_{o}$ is the outside radius of the tubes, $r_{i}$ is the inside radius of the tubes, $\mathrm{h}_{\mathrm{o}}$ is the inside heat transfer coefficient, $\mathrm{h}_{\mathrm{i}}$ is the outside heat transfer coefficient, and $\mathrm{k}_{\mathrm{ss}}$ is the thermal conductivity of stainless steel.

$$
\mathrm{Q}=\mathrm{U}_{\mathrm{o}} \mathrm{A}_{\mathrm{o}}\left(\mathrm{T}_{\text {Vapor }}-\mathrm{T}_{\text {Fluid }}\right)
$$

where: $\mathrm{U}_{\mathrm{o}}$ is the overall heat-transfer coefficient, $A_{o}$ is the outside surface area of the tubes, $\mathrm{T}_{\text {Vapor }}$ is the temperature of the vapor, and $\mathrm{T}_{\text {Fluid }}$ is the temperature of the fluid.

\subsubsection{Reactor Slurry Cooler Before The Centrifuge (HE-104)}

After the first run with coal and NMP, it was noticed that there needed to be a heat exchanger installed in between the reactor and the centrifuge. This heat exchanger was designed constructed and installed to reduce the temperature of the reactor's contents 
from $202^{\circ} \mathrm{C}$ to the maximum operational temperature of the centrifuge $\left(100^{\circ} \mathrm{C}\right)$ in less than 30 minutes. Figure 6-4 shows the operational temperature profile this heat exchanger.

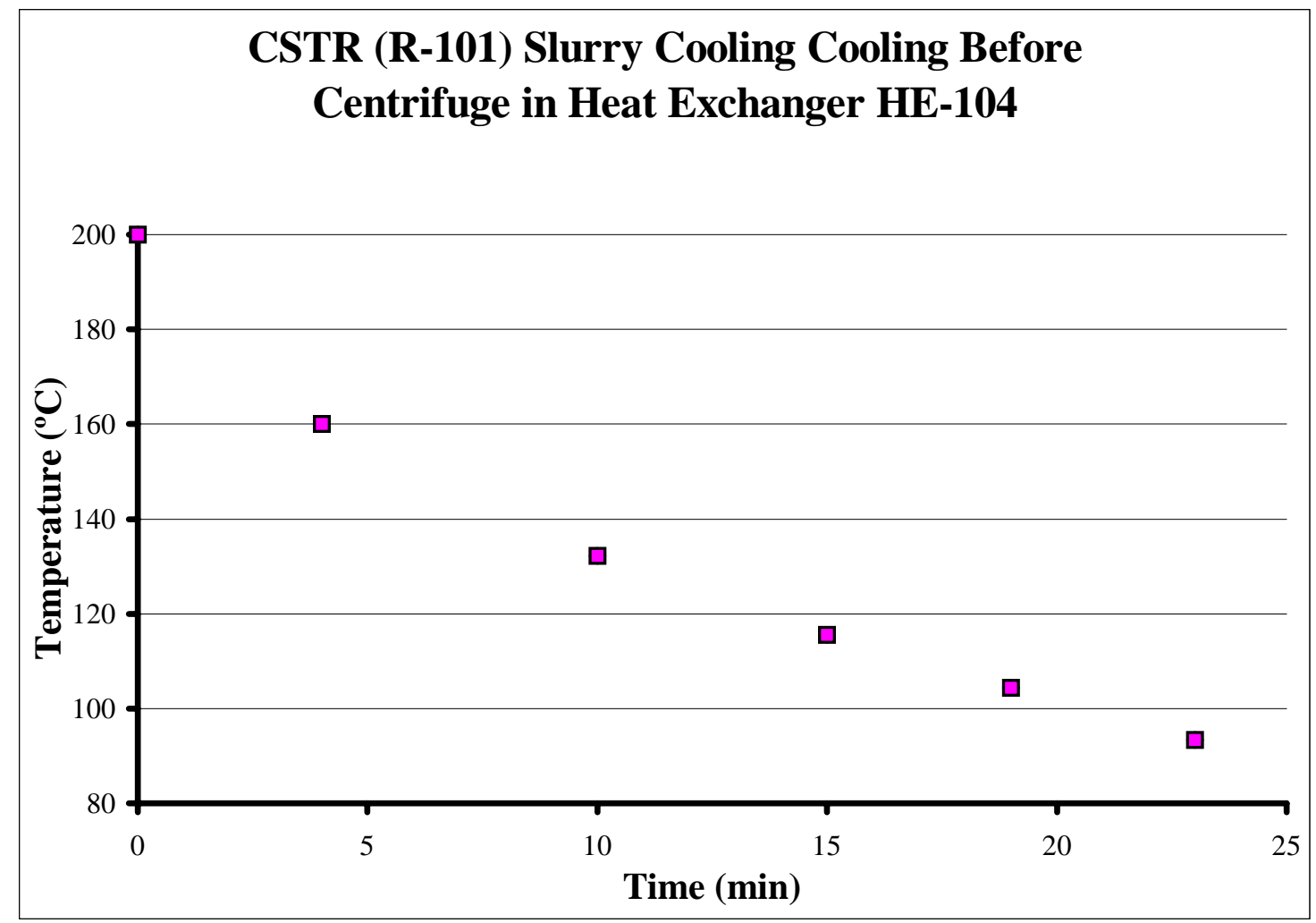

\section{Figure 6-4 CSTR (R-101) Slurry Cooling Before Centrifuge (Ct-101) With Supporting Heat Exchanger (HE-104)}

Heat Exchanger HE-104 was mounted vertically to ensure that the solids flowed from the top to the bottom. The tap water flowed on the shell side and the NMP/coal slurry on the tube-side. The overall average overall heat-transfer coefficient $\left(\mathrm{U}_{\mathrm{o}}\right)$ can be calculated for heat exchanger HE-104 cooling the NMP/coal mixture from the reactor using tap water. From Figure 6-4, the temperature is cooled from $392^{\circ} \mathrm{F}$ to $200^{\circ} \mathrm{F}\left(200^{\circ} \mathrm{C}\right.$ to $93^{\circ} \mathrm{C}$ ) in 23 minutes. Using Equation 6-1 to calculate the average specific heat of 2.1$\mathrm{kJ} / \mathrm{kg}{ }^{\circ} \mathrm{C}$, the true average rate of heat transfer $\left(\mathrm{Q}_{\text {avg }}\right)$ is $17.9 \mathrm{~kW}$. Substituting this into Equation 6-5, the average overall heat-transfer coefficient $\left(\mathrm{U}_{\mathrm{o}}\right)$ is calculated to be 230 $\mathrm{W} / \mathrm{m}^{2}{ }^{\circ} \mathrm{C}$. 


\subsubsection{Energy Balance on Wiped Thin Film Evaporator (TFE)}

(TFE-101)

A Wiped Thin Film Evaporator (TFE) was used as the third stage of a continuous process for making coal-based carbon pitches. The solvent (NMP) in the CSTR (R-101) dissolves the coal. Then the dissolved portion of the coal is separated from the undissolved portion in the Centrifuge (Ct-101). The TFE is then used to drive off the majority $(72 \pm 2 \%)$ of the solvent from the dissolved portion of the coal. Then a Rotovap is used to drive off the most of the remaining solvent from the product before it is placed in a vacuum drying oven to complete the process.

Again, the TFE evaporation system thickens the filtrate (solvent and dissolved coal) by separating a majority of the solvent through an evaporation process from the filtrate. Section 4.3.0 explains the operation of the TFE in great detail.

Section 3.2.4.3 details the five main operating parameters for the TFE that affect the quality of the product. Using the assumptions listed in section 3.2.4.3, four of the five operating parameters are established. The temperature of the heating fluid $\left(\mathrm{T}_{\mathrm{m}}\right)$ from the supporting heat exchanger (HE-102) is the only unknown parameter. An energy balance over the TFE for pure water can approximate this temperature.

The rate of heat transfer is needed to start the energy balance. Assuming that the inlet feed temperature $\left(\mathrm{T}_{\mathrm{in}}\right)$ is less than that of the saturation temperature governed by the operating pressure of the vacuum pump, the required rate of heat transfer is $3.1 \mathrm{~kW}$. This is for a feed rate $\left(\mathrm{F}_{\mathrm{in}}\right)$ of 0.5 liters per minute while assuming $72 \%$ of NMP is evaporated off. Next, the temperature of the filtrate film is needed to perform an energy balance over the system. Figure 6-5 show the log of the saturation pressure $\left(\mathrm{P}^{\mathrm{sat}}\right)$ of NMP vs. temperature $\left(\mathrm{T}^{\mathrm{sat}}\right)$. The saturation temperature is the temperature at which the NMP is boiling for a set pressure. This temperature is the temperature of the filtrate film. 


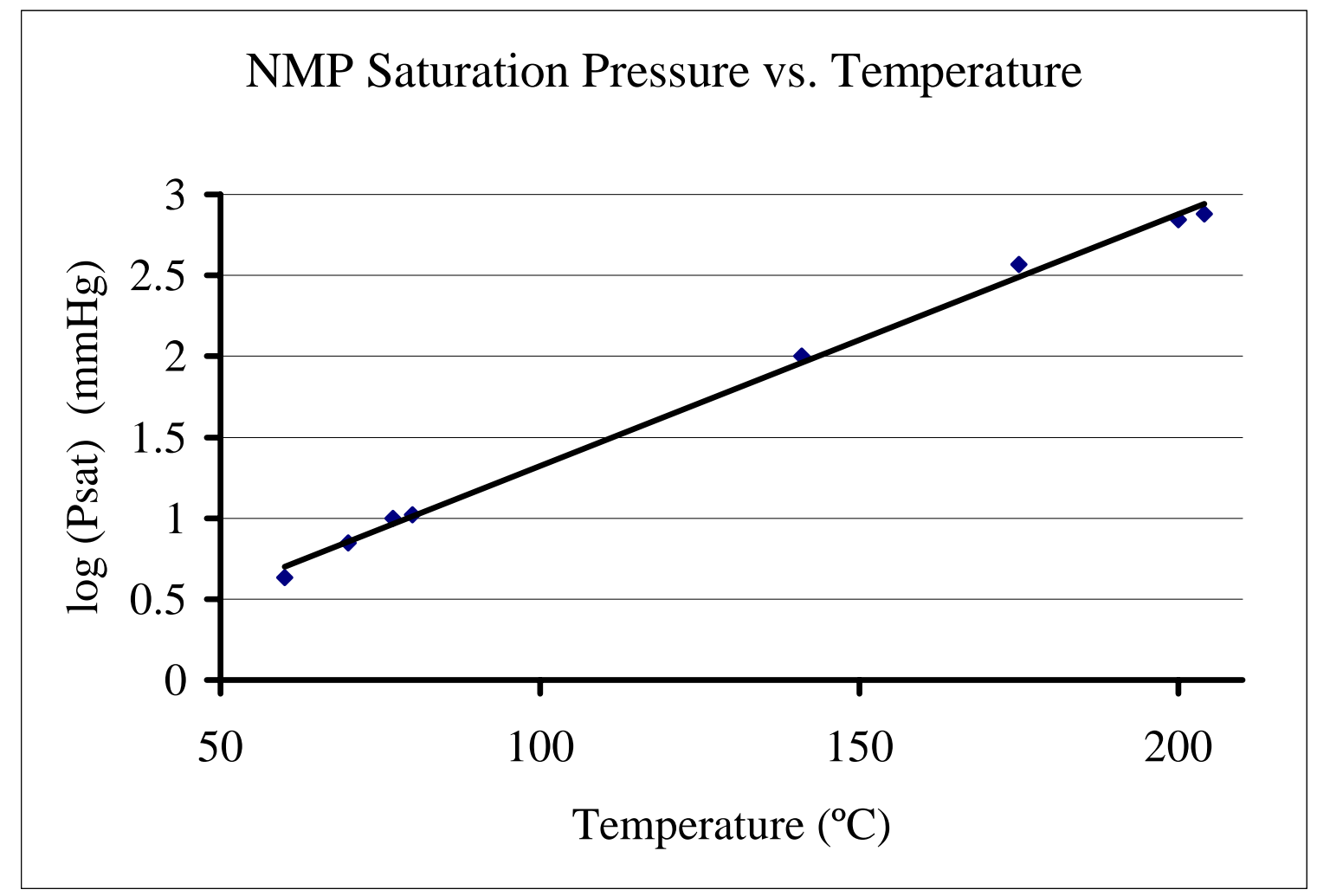

\section{Figure 6-5 Log of NMP Saturation Pressure vs. Temperature}

From Figure 6-5 the data are regressed to form the following equation for the saturation temperature $\left(\mathrm{T}^{\mathrm{sat}}\right)$.

$\mathrm{T}^{\mathrm{sat}}=\left\{\log _{10}\left(\mathrm{P}^{\mathrm{sat}}\right)+0.2349\right\} / 0.0156$

where: $\mathrm{T}^{\text {sat }}\left[{ }^{\circ} \mathrm{C}\right]$ is the temperature of the filtrate film in the TFE, and $\mathrm{P}^{\text {sat }}[\mathrm{mmHg}]$ is the operating pressure determined by the vacuum pump.

The maximum vacuum that can be drawn on the TFE by the vacuum pump (VP$101)$ is $5 \mathrm{mmHg}$. With this vacuum, Equation 6-6 gives a filtrate film temperature $\left(T_{\text {film }}\right)$ of $60^{\circ} \mathrm{C}$. Plugging the experimentally determined overall heat transfer coefficient for pure water and Marlotherm HT $\left(\mathrm{U}_{\mathrm{i} \text { water }}=150 \mathrm{~W} / \mathrm{m}^{2}{ }^{\circ} \mathrm{C}\right)$ into Equation 6-5, the calculated set operating temperature for the heating fluid $\left(\mathrm{T}_{\mathrm{m}}\right)$ for the supporting heat exchanger (HE-102) is $245^{\circ} \mathrm{C}$. 
The actual operating temperature was found to be $208^{\circ} \mathrm{C}$ leaving an $18 \%$ error. This error can be attributed to several sources: (1) the feed temperature for the NMP solution is at or above the film temperature in the TFE, while the feed temperature for the water case was atmospheric; (2) the feed rate for the NMP is about half that or plain water, (3) the higher operating temperature for the mineral oil could facilitate better heat transfer from the mineral oil, (4) the physical properties of water and NMP are very different, and/or (5) the supporting heat exchanger (HE-102) was larger than the one used in the water test, as a result it supplied the hot oil at a much higher rate.

The five operating parameters for a normal run of the TFE are shown in Table 61. These operating parameters are from runs 12 and 13. These two runs were processed together through the TFE at one time. The TFE is a continuous unit, and is not easily shut down. The TFE takes about an hour to start up and about an hour to clean between runs and prepare for the next. Therefore running two or more runs through at once helps to reduce down time. 


\section{Table 6-1 TFE Normal Operating Parameters}

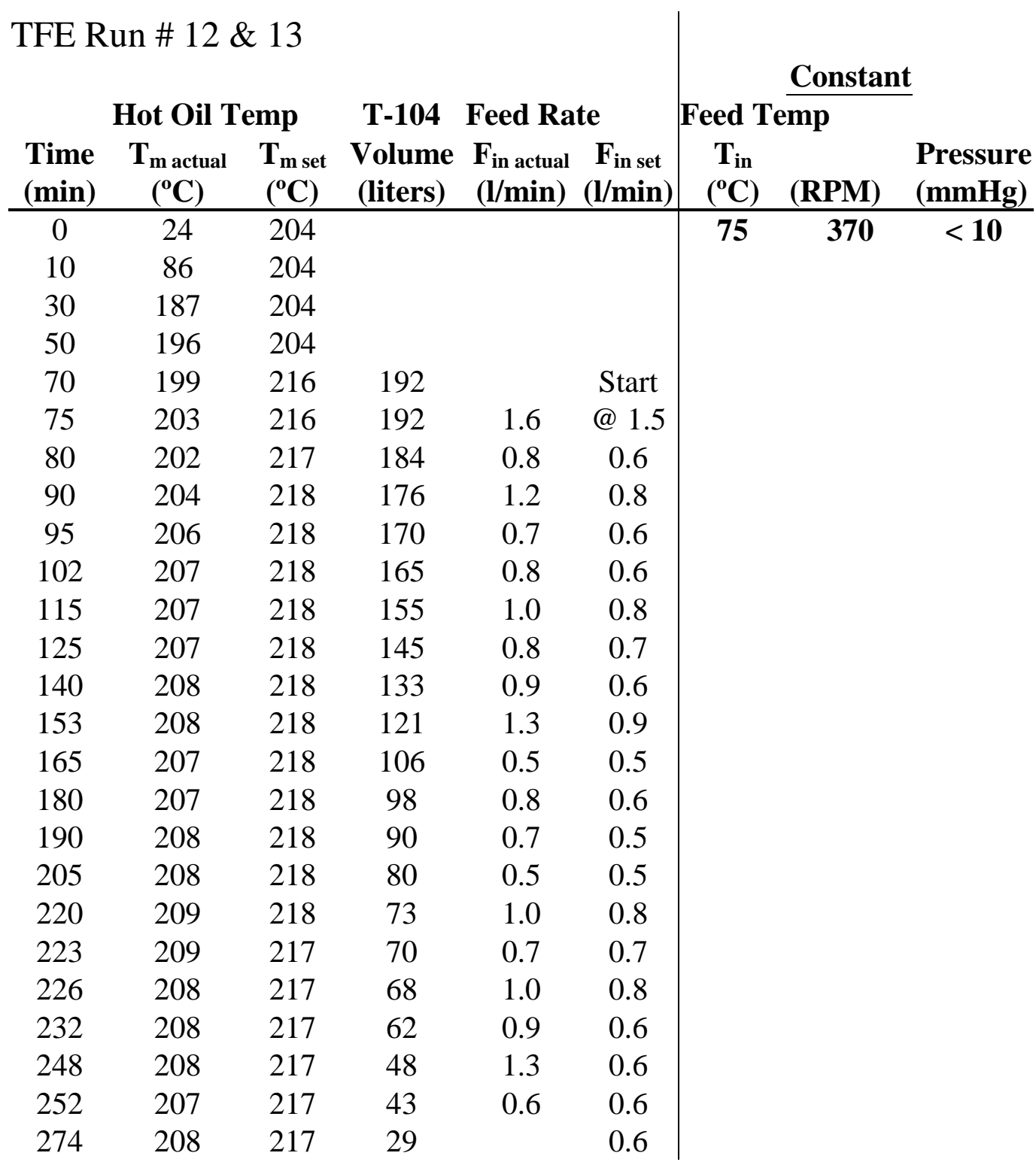

\subsubsection{Energy Balance on Rotary Evaporator (Rotovap)}

(RV-101)

The Rotovap operates much like a batch TFE. The Energy balance over this unit is fairly simple. The Rotovap takes the NMP concentration down from the 88-weight \% coming from the TFE to $50 \%$ by weight. The Rotovap processes 3 batches per run for a total of $3.5 \mathrm{~kg}$ extract and $25.5 \mathrm{~kg}$ of NMP. The total NMP driven off is $22 \mathrm{~kg}$ requiring 
a thermal load of approximately $12 \mathrm{MJ}$. The product, which is $50 \%$ product and $50 \%$ extract by weight, is then sent to the drying ovens.

The Rotovap has a condenser of $1.5 \mathrm{~m}^{2}$. This condenser is used to condense the majority of the vapors driven off. The Rotovap boils off NMP at a low temperature because of the low pressure pulled on the system by the vacuum pump (VP-101).

\subsubsection{Energy Balance on Drying Ovens}

(DO-101-3)

Again, the drying ovens are used to vacuum dry the coal waste leaving the centrifuge, and the semisolid extract leaving the Rotovap. The drying ovens essentially bake off the $55 \%$ by weight NMP in the processed coal stream, and the remaining $50 \%$ NMP in the product stream from the Rotovap. The coal waste stream leaving the ovens resembles that of the fresh coal entering the reactor. It is essentially unchanged other than the ash content. The product stream from the ovens is the coal-based carbon pitch in the form a dry friable granular powder.

The vacuum drying ovens use a nitrogen purge to carry the NMP vapors to the condenser (HE-105). Heat Exchanger HE-105 is the condenser for the drying ovens and vacuum trap for the vacuum pump (VP-102). This heat exchanger, shown in Figure 4-11, is made up of a 2-pass shell-and-tube heat exchanger mounted on top of a 20-gallon NMP condensate collection tank (T-106). This tank sets on top of a 45-gallon antifreeze sump (reservoir).

An air-operated diaphragm pump circulates the antifreeze from the 45-gallon sump through the tube side of the condenser (HE-105). This antifreeze is used as the cooling media to condense the NMP vapors that are driven off in the drying ovens. The NMP vapors leave the drying ovens and enter the freezer into the top left side of the collection tank. The vapor flows across the top of the collection tank and up into the condenser where it passes through a series of baffles. The vapor is condensed as it flows over the chilled tubes. The condensate flows counter currently down into the collection tank and is recycled to the reactor. The heat exchanger (HE-105), collection tank (T105), and antifreeze sump are contained in an upright freezer. 


\section{CHAPTER 7}

\section{RESULTS}

The Coal Extraction Pilot Plant was designed and built to allow the preparation of significant quantities of coal extract and evaluate the mass and energy balance of the process. The Upper Powleton coal (WVGS 1342 in these experiments is about $36 \pm 2 \%$ soluble in NMP during a 60-minute extraction period. As envisioned, $10 \mathrm{~kg}$ of coal is mixed with $100 \mathrm{~kg}$ of solvent in this experimental setup. Therefore, each run through the unit produces about $3.7 \mathrm{~kg}(8 \mathrm{lbs})$ of coal-based carbon extract.

Material and Energy balances have been calculated over the system. The question of solvent loss, or solvent retention, has been answered. Using the result of the Ultimate Analysis, there is a solvent retention of about $0.7 \%$ by weight of processed coal in the extract and coal. Assuming the worst case, all the lost solvent is in the final product, the extract contains less than $2 \%$ NMP by weight.

The Coal Extraction Pilot Unit is now being utilized to mass produce coal-based carbon extract and evaluate different operating processes and economics.

In conclusion:

1. The Coal Extraction Pilot Unit was built and operated efficiently. This process scheme was developed for the extraction and recovery of large amounts of coal extract from coal.

2. The final product (coal based carbon pitch, reconstituted coal, or extract) has a very low ash content $(<0.6 \%$ calculated from the ultimate analysis).

3. Solvent recovery has been addressed, and was found that grater than 99.9 $\%$ of the initial solvent was recovered.

4. By using the Ultimate Analysis in Appendix B, an overall material balance can be done over the system. This material balance shows a yield of $36 \pm$ $2 \%$, a yield (MAF) of $39 \pm 2 \%$.

5. The CEPU has a MBC of less than $1 \%$, and a solvent retention of $0.69 \%$ by weight of raw coal processed.

6. The TFE is not the best piece of equipment to be utilized in this process. The TFE would work better where there is a liquid/liquid separation. 
7. Water reduces NMP's ability to dissolve coal. As little a 3 mole \% (0.6 weight \%) will reduce the extraction yield to less than half.

8. The final reconstituted coal has an increase energy value of over 14,500 BTU/lb, as compared to the original Upper Powleton coal (WVGS13421), which has an energy value of 12,100 BTU/lb.

9. The final reconstituted coal contains $30 \%$ volatile matter, as compared to the original Upper Powleton coal, which has $26 \%$ volatile matter.

10. The final reconstituted coal has $68 \%$ fixed carbon, as compared to the original Upper Powleton coal (WVGS13421), which has fixed carbon value of $65 \%$. 


\section{CHAPTER 8}

\section{RECOMENDATIONS}

The most obvious question is: Why are all these steps required to remove the NMP from the coal extract? First, the TFE was designed to separate $70-75 \%$ volatile fluids from nonvolatile fluids. In this research, a volatile fluid was separated from a solid. In order for the TFE to operate continuously, enough NMP must be left in the "thickened coal extract" so it can flow. The effort is to minimize the amount of NMP in the coal extract and still have it flow. The rotary evaporator is designed to dry small quantities of material, which adhere to the walls of the container. When the coal extract dries, it no longer adheres to the container walls and a gum-like, thickened coal extract is produced. The material forms a large ball, limiting the effectiveness of drying with the Rotovap. The vacuum oven drying process is fairly slow-but very efficient.

It is possible that a single unit may be able to replace all three of the above drying units. Figure 8-1 and 8-2 show a double planetary mixer/vacuum dryer manufactured by Charles Ross and Son Company. This unit operates in a manner similar to a large Rotovap. However, instead of the vessel rotating in a hot oil bath, a set of blades (shown in Figures 8-3 and 8-4) in the vessel rotate and shear the thickened pitch (shown in Figure 8-5). The extract continues to dry until it starts to break apart forming a granular like that solid (shown in Figure 8-6). This solid is then wiped around until essentially all the NMP is driven off.

The walls of the planetary mixer are jacketed and heated by hot oil. The vessel is attached to a vacuum pump and condenser. The three drying steps in producing coal extract can be reduced into one. A single Ross double planetary mixer can perform the TFE, Rotovap, and drying oven steps. This use of this device greatly reduces process time and labor. Most significantly, it would minimize the need for physically handling the coal-based material. The efficiency of this device in this application was tested. The results from this test are included in Appendix D.

It must be remembered that only in the instances off solid/liquid separation does the TFE have problems. It is thought that the Rotovap and vacuum drying ovens will not be necessary for liquid/liquid separation. 
However, the purified coal extract has a softening point well above the boiling point of NMP. Therefore, the TFE has the potential of gumming up if too much NMP is driven off during the solid/liquid separation. Thus, two additional drying steps (Rotovap an drying ovens) are needed to produce the coal extract.

There were several other ideas brought up during the construction and operation of the CEPU that might enhance its performance. The following ideas should be considered.

- An up to date economic analysis for the proposed process needs to be done to further evaluate the economic feasibility.

- Centrifuge at a higher temperature and force. This is thought to remove more NMP from the coal waste, and increase the yield.

- Dry the coal before processing it. The less water put into the system the less that need to be removed. It would take less energy to dry the fresh coal than it would to clean the NMP up.

- Look at higher yielding coals. It only makes sense to get the most out of every run.

- Try dissolving the coal at a higher temperature (pressurized reactor). It is thought that this would increase extraction yields. The economics of the process may become more favorable as the percent extraction yield increases. The reactor needs only to be pressurized for this, and since the reaction steps involves only liquids, the cost of pumping would be minimal. The increase in percent yield due to more severe extraction conditions would outweigh the additional process costs.

- Look for a method for continuous drying large volumes or coal extract. The Charles Ross planetary mixer/vacuum dryer would only be suitable for smallscale production. For a larger scale facility, look at a Spray dryer, Falling Film dryer, Twin Screw Extruder vacuum dryer, ...etc.

- Examine the trace ash in the pitch to determine what it is and where it came from.

- Attention should be given to the possibility of reducing the solvent-to-coal ratio. Since the solvent represents the major cost of the process, such a reduction coupled with increased solvent recovery, would substantially alter the economics of the process. The minimum solvent-to-coal ratio that the CEPU can handle is not known. 
- The solvent retention should be determined through the use of radio labeled NMP or some other spectroscopic or analytical method. The past economic analysis has shown the process to be very sensitive to solvent retention (solvent loss). The present method of "back calculating " the level of solvent retention via the nitrogen balance that is derived from the ultimate analysis of the coal, residue, and extract, is at best a first approximation.

- Alternative uses for the waste coal (processed coal residue) should be investigated. A market, or use, should be found for this residue. Any profitable use would enhance the feasibility of the process. 


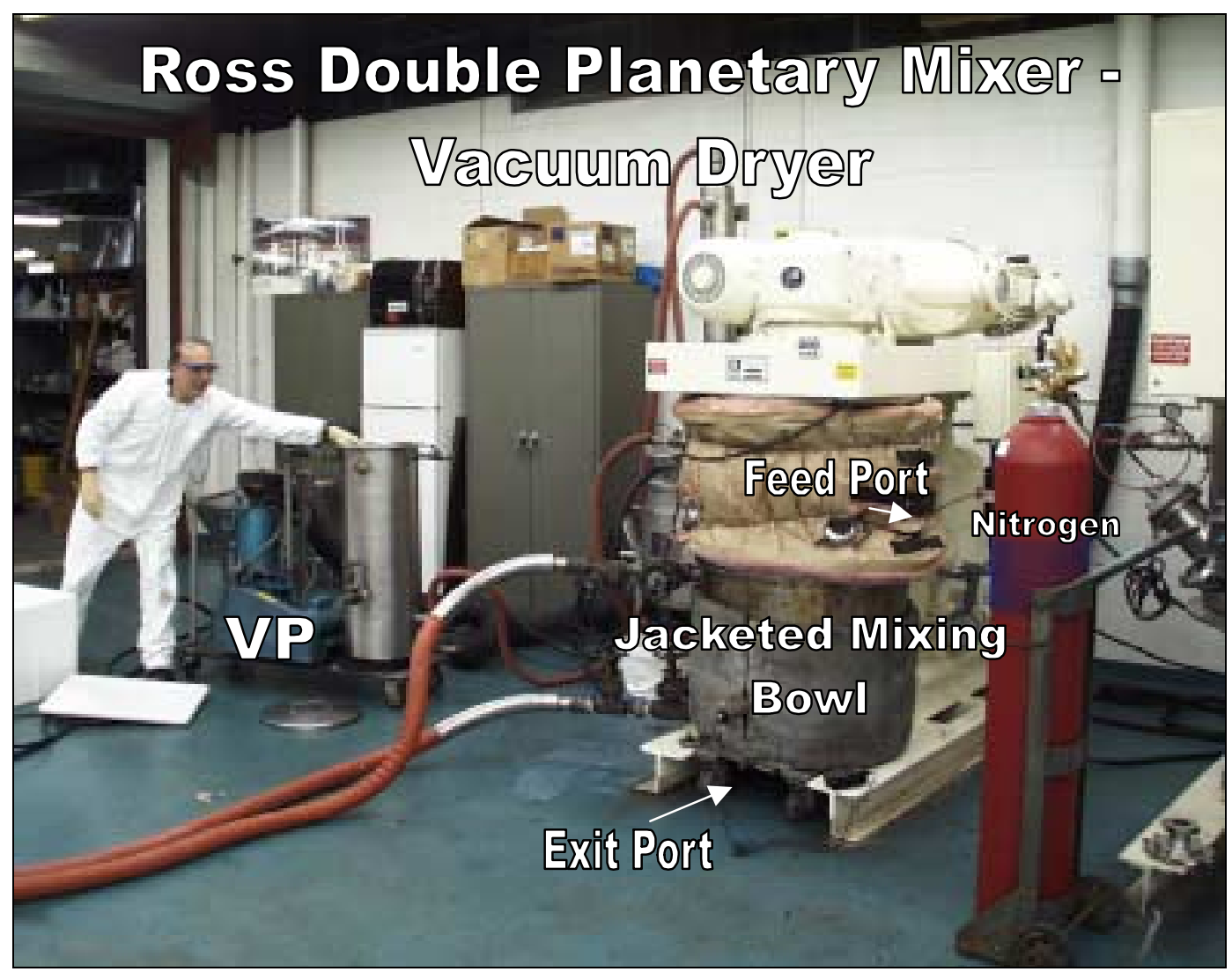

Figure 8-1 Charles Ross \& Son Co. (HDM-40)

40-gal Hydraulic Double Planetary Mixer

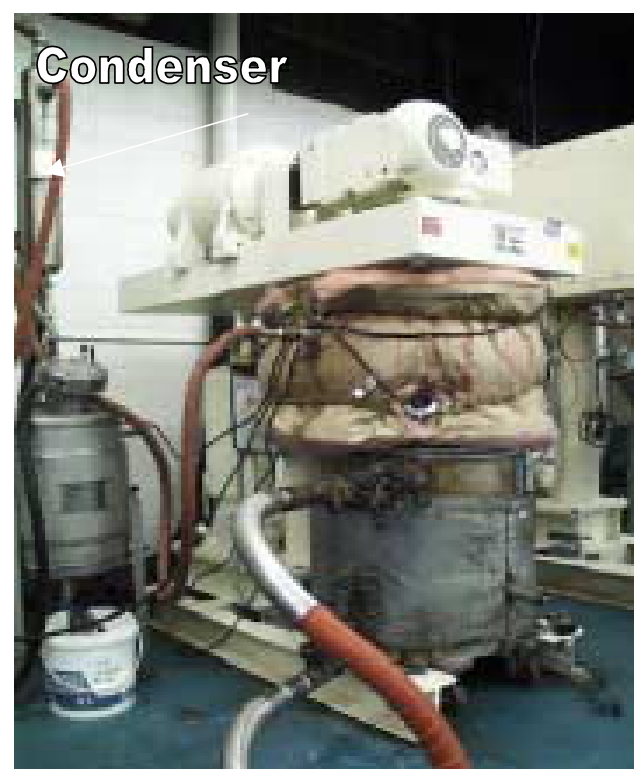

Figure 8-2 Hydraulic Double Planetary Mixer with Supporting Condenser 


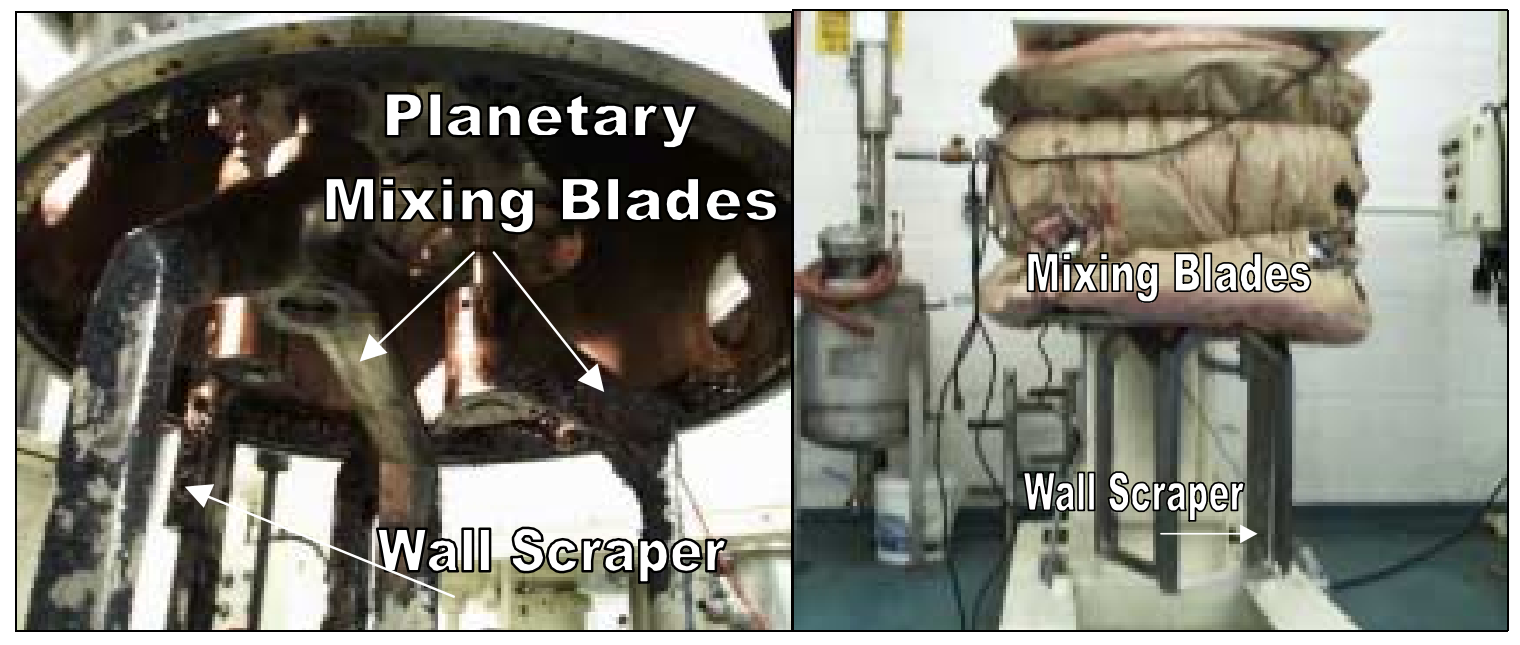

Figure 8-3\&4 Double Planetary Mixing Blades with Wall Scraper

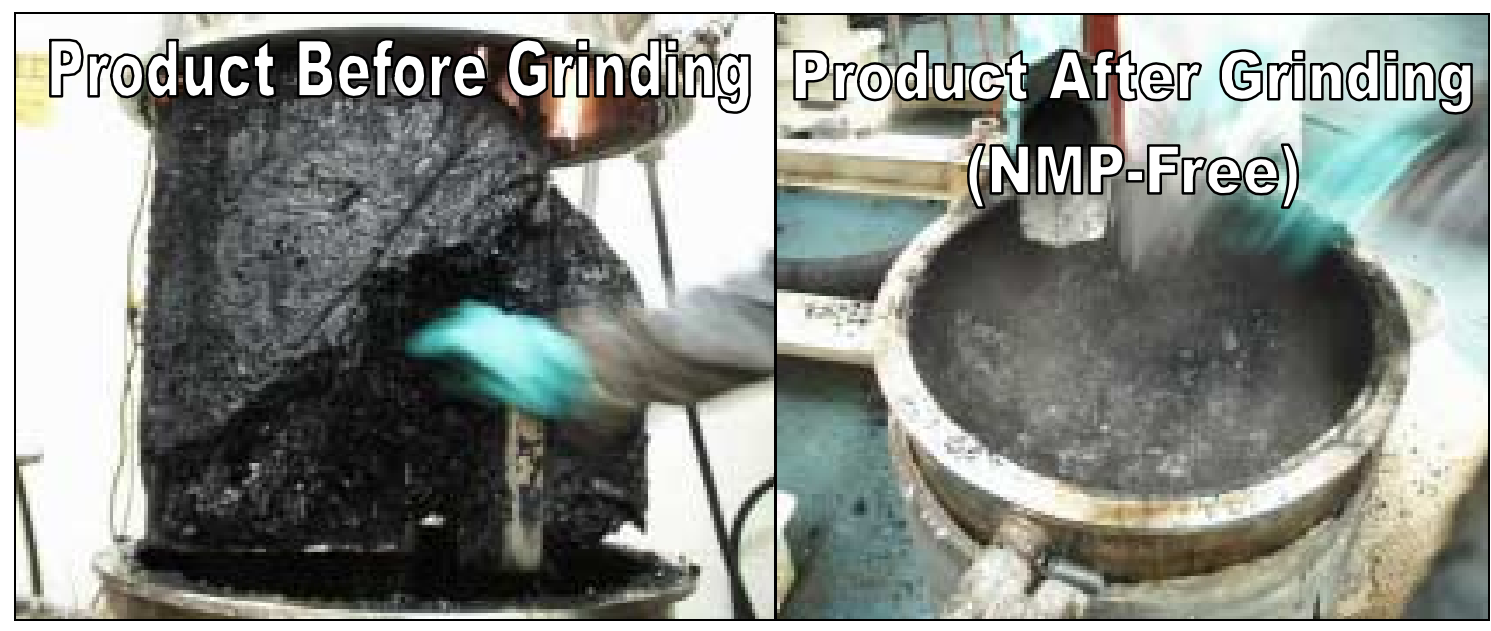

Figure 8-5\&6 Double Planetary Mixing Blades

With Product Before and After Grinding 


\section{REFERENCES}

American Society for Testing and Materials, Committee D-5 on Coal and Coke, ASTM Standards on Coal and Coke, American Chemical Society for testing and Materials, Philadelphia, PA 1984.

Asbury, R. S., Industrial Engineering Chemistry, Vol. 26, pg.1301, 1934.

Bakes, W. E., The Action of Solvents on Coals, Department of Scientific and Industrial Research, Fuel Research: Technical Paper No. 37, 1933.

Berkowitz, N., An Introduction to Coal Technology, New York, Academic Press, 1966.

Bonifera, V.P. Jr., Economic Analysis of Integrated Process or The Production of Coal Derived Carbon Products. Thesis, Department of Chemical Engineering, West Virginia University, Morgantown, WV 1997.

Dryden, I. G. C., Fuel, Vol. 29, pg197, 1950.

Dryden, I. G. C., Fuel, Vol. 30, pg39, 1951.

Dryden, I. G. C., Fuel, Vol. 34, pg383, 1954.

Eliot, R. C., Coal Desulfurization Prior to Combustion, Noyes Data Corporation, Park Ridge, New Jersey 1978.

Francis, W., Coal-Its Formation and Composition, Metcalfe \& Cooper, Ltd., London, 1954.

Gadam, S.D., Coal Upgrading Under Mild Conditions Using Supersolvents, Graduate Thesis, West Virginia University, Department of Chemical Engineering, Morgantown, WV 1990.

GAF Corporation, Chemical Division, M-Pyrol-N-Methyl-2-Pyrrolidone, New York, NY 1972.

Gerstner, J.A., Laboratory investigation, Design and Economic Assessment of a low Temperature Coal De-ashing and Desulfurization process, Thesis, Department of Chemical Engineering, West Virginia University, Morgantown 1989.

Gorbaty, L.M., Larsen, J. W., Wender, I., Coal Science, Academic Press, New York, NY 1982

Gorbaty. L.M., Ouchi, K., Coal Structure, American Chemical Society, Washington, D.C. 1981. 
Hensel, R. P., Coal: Classification, Chemistry, and Combustion, paper presented at CoalFired Industrial Boilers Workshop, Raleigh, NC, Fossil Power Systems, Combustion Engineering, Inc., Windsor, CT.

Holman, J. P., Heat Transfer, Eight Edition, McGraw-Hill, Inc. New York, NY 1997.

Illingworth, S. R., Fuel, Vol. 34, pg.363, 1922.

Kirov, N.Y., BCURA Monthly Bulletin, 1960.

Larsen, J.W., Organic Chemistry of Coal, American Chemical Society, Washington, D.C. 1978.

Leong, K., Coal Liquefaction at Mild Conditions Utilizing NMP and TMU Solvents, Undergraduate Thesis, West Virginia University, Department of Chemical Engineering, Morgantown, WV 1985.

Lowry, H. H., The Chemistry of Coal Utilization, Volume 1, John Whiley \& Sons, New York 1945.

Lowry, H. H., The Chemistry of Coal Utilization, Supplementary Volume 2, John Whiley \& Sons, New York 1962.

Marzec, A., Juwa, M., Betlej, K. an Sbokowwik, M., Fuel Processing Technology, Vol. 2, pp35-44, 1979.

Meyers, R. C. Coal Structure, Academic Press; New York, NY, 1982

Oele, A. P., Fuel, Vol. 30, pg.169, 1951.

Phillips, G.R., Solvent Extraction of West Virginia Coals in N-Methyl Pyrrolidone, Thesis, Department of Chemical Engineering, West Virginia University, Morgantown, WV 1988.

Renganathan, K., Beneficiation of Bituminous Coals Using Solvent Treatment At Mild Conditions, Thesis, Department of Chemical Engineering, West Virginia University, Morgantown, WV 1986.

Roy, J., Banerjee, P., and Singh, P. N., Indian Journal of Technology, 14, 1978.

Schobert, H.H., Coal-The Energy Source of the Past and Future, American Chemical Society, Washington D.C., 1987

Smith, K. L., Smoot, L. D., Flecher T. H., Pugmire, R. J., The Structure and Reaction Processes of Coal, Plenum Press, New York, NY 1994. 
Speight, J.G., The Chemistry and Technology of Coal, Marcel Dekker, Inc. New York, NY, 1983

Stewart, W.R., Design and Evaluation of a Batch Reactor System for Catalytic Coal Liquefaction, Thesis, Department of Chemical Engineering, West Virginia University, Morgantown, WV 1992.

Stiller, A. H., Sears, J. T., Hammack, R. W., United States Patent 4,272,356, June 9, 1981.

Stiller, A. H., Zondlo, J. W., Mintz, E. A. Jagodzinski, P. A. and Leong, C. W., "Interactions of solvents on Coal", Proceedings of the $2^{\text {nd }}$ Annual Pittsburgh Coal Conference, p413, Sept. 1985.

Stoppes, M. C., Wheeler, R. V., Fuel, 3, pg63, 1924.

Volborth, A., Coal Science and Chemistry, Elsevier Science Publishers B.V., New York, NY, 1987.

Van Krevelen, D. W., Coal Typology-Chemistry-Physics-Constitution, Elsevier Publishing Co., New York, NY, 1961.

Wise, W. S., Solvent Treatment of Coal, Crane Russak \& Co., New York, NY 1971.

Wiser, W., Preprints, American Chemical Society, Fuel Chemical Division, 1975.

Zondlo, J.W., Class notes ChE 224, Coal Processing, West Virginia University, Department of Chemical Engineering, Morgantown, WV Spring 1995.

Zondlo, J.W., Presentation to Dupont and New Car Carbon at West Virginia University about Coal Processing, West Virginia University, Department of Chemical Engineering, Morgantown, WV, July 2000. 
$\underline{\text { APPENDIX A }}$ 


\title{
Approval Sheet
}

for

\section{Experimental Usage in NR CCE}

\begin{abstract}
Applicant: Coal Based Carbon Pitch Team
Dr. D. Dadyburjor (ChE)

Dr. A. Stiller (ChE)

Dr. J. Zondlo (ChE)

Dr. P. Stansberry (ChE)

Dr. J. Plucinski (ChE)

Brian Bland (ChE)

Purpose: $\quad$ Erection and operation of a Carbon Extraction Pilot Unit (CEPU) within the confines of the NRCCE High Bay Laboratory Mezzanine. The pilot unit will produce coal based carbon pitch, which will be extracted from coal particles using N-methyl-2-pyrrolidone (NMP) as the extraction solvent. All procedures will initially be performed as batch unit operation processes involving various heat and pressure parameters. The processes may eventually be automated to allow for a continuous processing protocol. The equipment and processes will represent a scale-up from a laboratory bench scale to a small pilot scale.
\end{abstract}

Approvals:

NRCCE Safety Review: F. Saus

Date

Date

Chemical Hygiene Review: $\quad$ D. Brant

Date

WVU ES\&H: M. Naugle

Date

Director NRCCE: R. Bajura 


\section{COAL EXTRACTION PILOT UNIT}
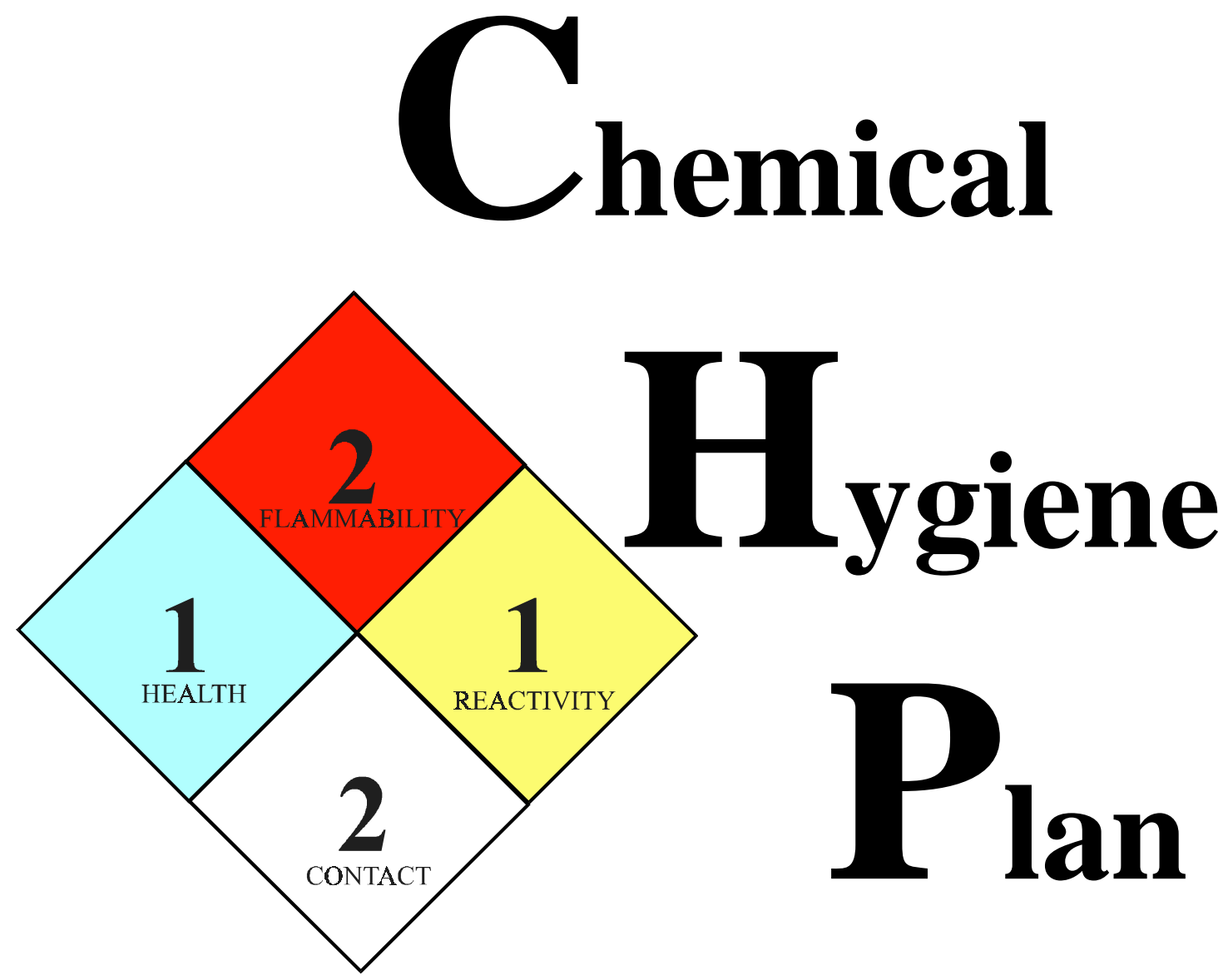

NFPA Chemical Hazard Label (explained on page A-3)

\begin{tabular}{lll}
\multicolumn{2}{l}{ EMERGENCY TELEPHONE NUMBERS: } \\
\hline Brian Bland & Office: & $\mathbf{2 9 3 - 2 1 1 1}$ ext. 2423 \\
& Home: & $\mathbf{8 2 5 - 6 6 4 2}$ \\
Al Stiller & Office: & $\mathbf{2 9 3 - 2 1 1 1}$ ext. 2408 \\
& Home: & $\mathbf{2 9 6 - 1 8 7 3}$ \\
Frank Saus & Office: & $\mathbf{2 9 3 - 7 3 1 8}$ ext. 5440 \\
& Home: & $\mathbf{5 9 9 - 1 2 5 6}$
\end{tabular}




\section{EMERGENCY SHUTDOWN PROCEDURE}

The following procedure will correctly shut down the equipment in the event of an emergency and/or if the operator is unable to be found or is found unconscious at the site.

Press in upon one of the two labeled Emergency Shut Off Button (Shunt Trip) located at the entrance way [top of the stairs] or at the emergency exit doorway of the mezzanine. Depressing either of the two buttons will eliminate, electrical power being applied to the equipment. The buttons are clearly signed and marked for immediate recognition.

Following shut down of electrical power to the equipment, carefully survey the area for injured or unconscious victims. If found, immediately dial 911 and secure outside assistance.

If no individuals are on the premises, carefully assess the experimental area and make remote visual inspection for any hazards such as unstable objects, fire, liquid being lost by the equipment, etc. Contact Security at 911 and appropriate individuals from the calling list as necessary.

\section{SEE DRAWING IN FIGURE A-1 FOR THE LOCATION OF THE EMERGENCY SHUT OFF (SHUNT TRIP) BUTTONS.}




\section{NFPA Chemical Hazard Label}

NFPA Rating - The National Fire Protection Association (NFPA) has developed a standard system (ANSI/NFPA 704) for indicating the health, flammability, and reactivity hazards of chemicals. In addition, a special precaution symbol may be used where necessary.

This system of identifying hazards associated with various materials was developed primarily for fire protection and emergency personnel but can be useful to anyone who needs to handle potentially hazardous material. As stated in NFPA 704, "This standard provides a simple system of readily recognizable and easily understood markings, which will give at a glance a general idea of the inherent hazards of any material and the order of severity of these hazards as they relate to fire prevention, exposure, and control".

\section{General Rating Summary}

\section{Health (Blue)}

4 Danger May be fatal on short exposure. Specialized protective equipment required

3 Warning Corrosive or toxic. Avoid skin contact or inhalation

2 Warning May be harmful if inhaled or absorbed

1 Caution May be irritating

$0 \quad$ No unusual hazard

\section{Flammability (Red)}

4 Danger Flammable gas or extremely flammable liquid

3 Warning Flammable liquid flash point below $100^{\circ} \mathrm{F}$

2 Caution Combustible liquid flash point of $100^{\circ}$ to $200^{\circ} \mathrm{F}$

$1 \quad$ Combustible if heated

$0 \quad$ Not combustible

\section{Reactivity (Yellow)}

4 Danger Explosive material at room temperature

3 Danger May be explosive if shocked, heated under confinement or mixed with water

2 Warning Unstable or may react violently if mixed with water

1 Caution May react if heated or mixed with water but not violently

0 Stable Not reactive when mixed with water.

\section{oWhite}

Special Symbols

\section{Contact Mild irritant}




\section{COAL EXTRACTION PILOT UNIT -- CEPU}

This document advances the placement, erection and operation of a Coal Extraction Pilot Unit (CEPU) within the confines of the NRCCE High Bay Laboratory Mezzanine. The pilot unit will produce coal based carbon pitch, which will be extracted from coal particles using N-methyl-2-pyrrolidone (NMP) as the extraction solvent. All procedures will initially be performed as batch unit operation processes involving various heat and pressure parameters. The processes may eventually be automated to allow for a continuous processing protocol. The equipment and processes will represent a scale-up from a laboratory bench scale to a small pilot scale. This document calls out the necessary protocols to meet the safety, health and chemical hygiene requirements necessary to protect researchers, operators and student workers as stipulated under Federal, State, Local, and University mandates, requirements, statutes and ordinances.

\section{Presumptions}

1. The requesters will be the sole occupants and users of the NRCCE High Bay Mezzanine area in order to conduct their operations, which will include the construction, and operation of the CEPU.

2. Due to the nature of the operational protocol, only necessary personnel will occupy the Mezzanine area during operation of the equipment. There will always be two (2) qualified operators present during the operation to ensure that an equally knowledgeable back-up individual is present to support the prime operator in the event of any perturbations from the reactions or mechanical operation of the equipment.

3. All equipment when not in operation will be placed on "lock out" mode with one set of keys being given to the Safety Officer of NRCCE.

4. All process reactants, products and wastes will be maintained in sealed metal or plastic drums and/or stored in appropriate devices as designated for respective material/chemical containment.

5. Operators will be solely responsible for maintaining clean and safe area including the removal of all waste and trash generated by operators or the unit. Custodians will not be permitted to enter the area once the unit starts to be constructed.

6. All operators will be trained in the use, inspection and operation of the 10-Ton overhead crane by appropriate ES\&H personnel. In addition, all personnel who will come into contact with reactants, products or waste substances classified as hazardous materials will be required to undergo the appropriate training as designated by the NRCCE Chemical Hygiene Officer (CHO), the WVU Environmental Safety and Health office (ES\&H) or meet 29 CFR1910.720 HAZWOPR standards. Contact will entail activities such as receiving or shipping 
of drums, materials handling or operation of equipment associated with the pilot unit.

7. Prior to any experimentation, researchers and operators in conjunction with the NRCCE CHO and the office of WVU ES\&H will stipulate the appropriate Personal Protective Equipment (PPE) to be used in conducting any experimentation or operation of the CEPU.

\section{Approach to Design}

Engineering controls are the most effective way of reducing or eliminating exposure to chemical or physical materials and/or conditions. The solvent used in the extraction process, N-methyl-2-pyrrolidone (NMP), is classified as a dipolar aprotic organic solvent. NMP is only hazardous under abnormal conditions. On a hazard scale from 0 to $4(0=$ no hazard, $4=$ extreme hazard $)$, NMP is given a 2 for flammability, 2 for contact, 1 for health, and 1 for reactivity on the Baker Safe-T-Data (TM) Precautionary Labeling System (see MSDS sheet attached).

NMP will be contained and recycled within the system limiting any direct contact exposure during loading, unloading, or transferring processes, as well as, restricting the opportunity for any potential leaks. All equipment with the potential to produce any NMP vapors will be exhausted to the outside, eliminating exposure to gaseous vapors. Any dissipation of heat produced by the operating equipment will be mitigated by convection into the Mezzanine's special area and removed by the buildings exhaust fans if necessary.

The best available technology for providing personal safety protection is containment or enclosure of all operations and provision of local exhaust ventilation at the site of chemical release. The CEPU has been inherently safely engineered to keep NMP (liquid or vapors) away from any extreme heat, sparks, and flame; and avoid contact with eyes, skin, and clothing. While use of personal protective equipment (PPE) is generally considered less effective than engineering controls, they will be utilized in the proposed operation as an additional level of safety measures for the production of the coal based carbon pitch.

The following approach was used to evaluate the engineering controls:

1. How hazardous is the substance?

2. How much of the substance will be released?

3. Will any release be harmful to skin or eye contact?

This document will at times address a minimum condition for the Mezzanine environment and a maximum condition in order to properly address the degree of severity of any contaminant. The minimum conditions are more relevant to the proposed experimentation, while a maximum condition may be required to meet higher contaminant levels or future protocol scenarios such as continuous and batch operations.

\section{Overview of the Unit Processes}


The accompanying process flow diagram (figures A-2\&3) is representative of the processes to be used and afford some preliminary conditions for operation. A stream table follows in table A-2, and a equipment description in table A-3.

** "Product" refers to the soluble fraction of coal, or coal based carbon pitch.

** "Waste" refers to the insoluble fraction of coal.

** "NMP" refers to the virgin or recycled solvent.

"Solid Liquid Slurry" refers to a mixture of soluble and insoluble coal in NMP. That is a mixture of product, waste, and NMP.

Extraction Unit (R-101): The extraction process will be accomplished by loading fine particle coal (70 microns screened) and NMP into the extraction unit. The "solid liquid slurry" will be heated to $202^{\circ} \mathrm{C}$ and maintained for one hour with mixing. Any vapors generated in the reactor will be condensed by Heat Exchanger (HE-101). The solid liquid slurry will then be cooled to $100^{\circ} \mathrm{C}$ before further processing by heat exchanger HE-104.

Slurry Pump (P-101): After cooling, the solid liquid slurry will then be pumped to the centrifugation unit (Ct-101) by a variable speed gear pump.

Centrifugation Unit (Ct-101): Once loaded into the continuous centrifugation unit, the solid liquid slurry will be separated into an insoluble "waste" (stream 15) and a soluble "product-NMP" mixture (stream 7).

The "waste" (15) component will be treated as a hazardous material. This "waste" will be transferred to a vacuum drying oven (DO-101).

The "product-NMP" (7) mixture will be stored in tank (T-101) to a wait processing by the Thin Film Evaporation unit (TFE-101) for removal of the majority of "NMP" from the dissolved or suspended "product".

Pump (P-102): Then, the "product-NMP" mixture will be pumped from tank (T101) to the Thin Film Evaporator (TFE-101) by a variable speed gear pump.

Thin Film Evaporator (TFE-101): Utilizing a supporting heat exchanger (HE 102) and a cooling water condenser, the "product-NMP" mixture will be further partitioned into two components under a vacuum by the evaporation unit.

A minimum $50 \%$ of the "NMP" will be evaporated off the mixture of "product-NMP" and condensed using the internal cooling water condenser. This "NMP" will be removed as a pure liquid and stored in a tank (T-103) to be recycled back to the extraction unit by the recycle pump (P-103).

A second stream of "product" and reduced "NMP" mixture will be removed from the evaporation unit and stored in a tank (T-102). The sludge like 
"product-NMP" mixture will be further refined using a Rotary Evaporation Unit (RV-101) to isolate the desired carbon "product".

Heat Exchanger (HE-102): The heat needed to evaporate the "NMP" from the "product" in the Thin Film Evaporation unit (TFE-101) will be supplied by a $20 \mathrm{~kW}$ heat exchanger using mineral oil as the transfer media.

Vacuum Pump (VP-101): A 755-mmHg vacuum will be drawn on the evaporation unit using a dual sealed Welch Vacuum Pump. The off gas will be exhausted to the outside from the Mezzanine.

Heat Exchanger (HE-103): Any NMP vapors escaping the internal evaporation unit's condenser will be condensed and trapped by this Vapor Trap in line just before the Vacuum Pump (VP-101).

Recycle Pump (P-103): "NMP" will be recycled from storage tank (T-103) to the extraction unit (R-101) by a gear pump.

Rotary Evaporation Unit (RV-101): The remaining "NMP" concentration contained in the coal based carbon "product" after the Thin Film Evaporator (TFE-101) will be further reduced by distillation under a vacuum in Rotovap (RV-101). The recovered "NMP" will be recycled to the extraction unit.

Finally, the "product" will then be placed in a drying oven (DO-101) for two days at $130^{\circ} \mathrm{C}$ to remove any trace of "NMP". The finished product will then be placed in plastic bottles and stored in a freezer in the Engineering Research building at WVU until removed needed.

Rotary Evaporation Unit (RV-101): Any remaining "NMP" contained in the waste (insoluble coal) after the Continuous Centrifuge Unit (Ct-101) will be distilled off under a vacuum using Rotovap (RV-102).

The "waste" will then be drummed or sealed in pails and removed from the operational area for Hazardous Waste disposal, and the "NMP" will be recycled back to the extraction unit.

Vacuum Pump (VP-102): A 755-mmHg vacuum will be placed on the Rotovap to extract and recycle the "NMP". The off gas will be exhausted to he outside from the Mezzanine.

\section{Receiving and Shipping}


The user will enter into agreement with the supplier/vendor as to how the material will be packaged for shipment to NRCCE, as well as, packaging and labeling of used and unused materials for return to the supplier or for product and waste removal. If so stipulated and deemed necessary by the chemical material's concentration, all transportation of said materials must meet Department of Transportation Standards for Hazardous Material as prescribed by Title 49 CFR parts 107, 171-180, and 309-397. The amount of hazardous material, which will remain or be stored on site at NRCCE, is to be limited to $4-55$ gal drums.

Drums are to be sealed and stored in appropriate containment devices as addressed in Item 4. Drums of Waste Materials are defined as including drums containing coal/NMP unreacted material, product or NMP, as well as, drums containing expendable items such as gloves, clothing, buckets, shovels, etc. which may have been exposed to hazardous contaminants. The user will make arrangements with any supplier and/or the WVU ES\&H for routine disposal of expendable supplies, such as plastic wrap, plastic absorbent paper, used spill kit materials, hazardous suits, chemical paraphernalia, etc. generated during the experimentation or operational period.

1. Upon arrival of container(s) at the NRCCE loading dock, only trained individuals may take receipt of the container(s). The individual receiving the shipment will have on the same operational PPE required for the CEPU. The inspection of the container will be performed prior to removal from the delivery vehicle. The container will be surveyed for all appropriate labels and placards pertinent to the contents. The container upon inspection may be rejected and returned to sender, if the integrity of the container is suspect. If inspection of the container reveals that the integrity of the container could result in the exposure of the shipper to the hazard, the container should be removed and placed in a secure containment vessel or over-pack container prior to making arrangements with the sender for return of the damaged vessel.

2. The container will be removed from the delivery vehicle to the outer loading dock area utilizing a hydraulic pallet mover, a pallet drum truck or drum truck. The container will immediately be removed from the loading dock and transported to the high bay for appropriate storage or placed directly into the CEPU. 
3. The user will provide two Containment Systems: one for holding drums of fresh material and one for holding waste materials. The user may store 2 - 55 gal containers of fresh material and 2 - 55 gal containers of waste materials in the high bay or in a secured container on the loading dock for one month. Sealed containers may be stored on a Modular Poly Deck Safety Pack, plus tertiary containment system with a capacity of 2 or 4 drum containment. There should be separate, and labeled, containment systems for new and waste containers.

4. Transport of all reactants, supplies, product and waste will be removed from or delivered to the Mezzanine via a Swing Gate Forklift Platform on which the drums are secured, or a Drum Lifter Tilter in which the drums are secured. The platform, or lifter, will be raised or lowered from the Mezzanine or High Bay floor using the $10 \mathrm{~T}$ overhead crane operated by a trained operator.

\section{Storage and Waste Disposal Areas}

** The following assumes that hazardous materials will be contained in drums.

** "Poly Deck" (PD) is a modular spill deck, which can hold 2 or 4 drums, contain $17 \frac{1}{2}$ to 35 gallons of spillage, respectfully, and can accommodate a metal ramp to facilitate the addition or removal of drums from the deck. Design of the storage platform limited to two drums of fresh NMP and 2 drums of waste disposal materials.

1. Tools used for material handling should be kept clean, either by using disposable tools, wiping tools down with absorbent paper, or have a cleaning bucket for rinsing tools prior to storage. Tools used to handle the material at various stages of experimentation should be stored within or adjacent to their respective use area where drips and dust can be contained. Disposal should be in a plastic-lined drum for easy handling and containment of contaminated materials or apparatus as required.

2. Designated and correctly labeled drums will reside at the Waste Disposal deck for receiving various types of wastes, such as coal/NMP mixture from the centrifuge, glassware, tools, disposable outer garments, paper towels, etc.

3. Drums containing waste materials or contaminates will be correctly labeled and removed from the mezzanine to the containment systems for disposal, as soon as possible. 
4. All expendable supplies and equipment used in the parameter testing area will be brought onto the mezzanine as needed and be immediately removed or discarded after final use. Plastic, glassware and paper volume may be reduced using a dedicated trash compactor. Disposable containers or plastic lined containers will be placed under the bench tops to receive used apparatus or supplies.

\section{Processing Explanation and Operation}

\section{Extraction}

Material Transport

NMP will be stored in a designated ventilated Flammable Storage Cabinet in 2 sealed 55 gal containers on the Mezzanine. Coal will be stored at a designated area in sealed plastic bags. The NMP will be loaded into the extraction unit by the recycle pump or manually from the storage drums until 25-35 gallons are in place. Loading of the coal will be accomplished manually from sealed plastic bags until 10-20 pounds are contained in the extraction unit. Once in the extraction unit, all future material transport of through the processing equipment will be handled predominately by low rate gear pumps.

During manual loading, unloading, and storage operation, the containers for the coal, coal waste, and NMP will be located upon a secondary containment system. The secondary containment system for the CEPU is composed of Modular Poly Spill Decks linked together to capture any spills generated during the transfer operations from the bags, drums, tanks, ... etc. into, or from, any piece of equipment. With the spill deck linked together the sump capacity increases to meet the 40 CFR 264.175 [1996] capacity requirements. A tertiary containment system, composed of a Polyethylene Liner will also be in place to capture any spills escaping the primary containment system (see page 14 for placement secondary and tertiary containment systems).

\section{Operation Parameters}

Estimated Stream properties can be seen on page 18.

The Extraction Unit (R-101) will be inspected for loose fittings, screws, seals, wires, and belts prior to loading the unit with coal and NMP. Once loaded into the extraction unit, the controls will be set to heat the reactants will be up to $200^{\circ} \mathrm{C}$ using an electric heater. The unit contains a stirring mechanism, and water-cooled condenser that will operate during the extraction process. 
After two (2) hours of heating and mixing, the heating operation will be discontinued and the mixture allow to cool to $100^{\circ} \mathrm{C}$ by natural heat radiation loss through the unit's walls.

The Centrifugation Unit (Ct-101) will be inspected for loose fittings, screws, seals, wires, and bolts prior to loading the unit with the coal/NMP Slurry from the Extraction Unit. Once loaded into the continuous centrifugation unit, the solid liquid slurry will be separated into a insoluble waste component and a soluble liquid component (product dissolved in NMP) under a $3000 \mathrm{~g}$-force.

Thin Film Evaporator (TFE-101) will be inspected for loose fittings, screws, seals, wires, and bolts prior to loading the unit with the product and NMP mixture from the Centrifugation Unit. The productNMP mixture will be further partitioned into two components, under a $755-\mathrm{mmHg}$ vacuum, by the evaporation unit. Utilizing a supporting heat exchanger, and a water-cooled condenser, at least 50\% of the NMP will be evaporated off and condensed using the internal cooling water condenser. This NMP will be removed as a pure liquid and recycled back to the extraction unit. A second stream of a product and reduced NMP mixture will be removed from the evaporation unit to be further refined using a Rotary Evaporator and a Drying Oven.

Pressure release valves will be in line just after Gear Pumps (P$101,2, \& 3)$ with a Set Pressure at $10 \%$ over Maximum Operating Pressure of the weakest vessel and/or line.

Vacuum Pumps (VP-101-2) will draw a 755-mmHg vacuum. The off gas from these vacuum pumps along with any vapors generated by the extraction unit (R-101) will be vented through a ductwork to the positive exhaust system of the building.

Rotary Evaporators will operate under a $755-\mathrm{mmHg}$ vacuum. Bath oil will bring the mixture containing NMP up to $1400^{\circ} \mathrm{C}$. Cooling water at approximately $18^{\circ} \mathrm{C}$ will be used to condense the NMP to be recycled back to the Extraction Unit.

The operation area will be checked for spills, loose fittings, screws, seals, wires, and belts prior before unlocking the CEPU lockout. The operation area is to be secured and power unit placed in Lock-Out Tag Out before it is left unaccompanied.

\section{Safety Requirements \& Concerns}


** "Dipolar" refers to the separation of positive and negative charges that occur on the molecular structure.

** "Aprotic" refers to a solvent with no proton available for hydrogen bonding.

Coal

The coal will be screened to 70 microns, but will most likely also contain smaller particulates due to attrition. Preliminary examination shows that the coal contains less than $3 \%$ particulates less than 10 microns. A respireable dust mask will be worn during the loading operations.

NMP

NMP is abbreviation for N-Methyl-2-Pyrrolidone. NMP is designated as a dipolar aprotic organic solvent. NMP is moderately flammable and a slight contact irritant. NMP is only hazardous under abnormal conditions.

Precautionary Labeling (MSDS)

Baker Safe-T-Data (TM) System

\begin{tabular}{|c|c|c|}
\hline Health & -0 & None \\
\hline Flammability & & Modera \\
\hline Reactivity & - & None \\
\hline Contact & - & Slight \\
\hline
\end{tabular}

Hazard Ratings are $0-4(0=$ No Hazard; $4=$ Extreme Hazard $)$

The CEPU is designed so that NMP is contained and recycled within the system limiting any direct contact exposure during loading, unloading, or transfer processes, as well as, restricting the opportunity for any potential leaks. All equipment with the potential to produce any NMP vapors will be exhausted to the outside by ductwork and a exhaust fan, eliminating exposure to or build up of gaseous vapors. The dissipation of any heat produced by the equipment is another safety consideration for the NRCCE Laboratory Mezzanine. The Mezzanine's immense volume and four enormous exhaust fans are more than adequate to eliminate this problem.

The potential hazard with skin and eye contact is diminished by requiring the proper PPE of gloves, lab coats or coveralls, and safety glasses or face shields discussed in item \# 7 , to protect individuals from leaks, spills, etc. In the event of any contact, an Emergency Safety Shower and Eye Wash Station are available and labeled. A emergency response sheet will be posted as to whom to call with appropriate telephone numbers. 
Dry Chemical Fire Extinguishers rated for class A, B, and $\mathrm{C}$ fires will be conveniently located in the event of a fire. Emergency shut off buttons and fire alarms are to be labeled and located next to the emergency exits. Emergency Spill Kits will be available for spill containment.

Coal Based Carbon Pitch

The desired extract, or pitch, is refined ashless coal. This pitch has not been chemically altered in any way by this process (see attached publication for bio-reactivity of novel carbonaceous pitches).

Coal Waste

All traces of NMP will be removed for the coal waste (insoluble coal) before disposal. The coal waste has not been chemically altered in any way by this process. This waste is essentially inert coal with a higher ash (mineral) content.

Equipment

Equipment will be operated by control panels, located at a safe distance, keeping personnel away from any moving parts such as belts, motors, stirrers, pumps, etc. The entire CEPU will have a Lock-Out Tag-Out power unit with only one key. While not in operation, the unit will be placed on "lock out" mode with one set of keys being given to the Safety Officer of NRCCE (item \# 3).

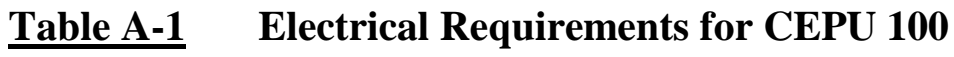

\begin{tabular}{|c|c|c|c|c|}
\hline UNIT \# & DESCRIPTION & VOLTAGE & PHASE & $\underline{\mathrm{AMP}}$ \\
\hline 1R-101 & Extraction Unit & 220 & 1 & 60 \\
\hline Ct-101 & Continuous Centrifuge & 230 & 3 & 21 \\
\hline TFE-101 & Thin Film Evaporator & 230 & 3 & 7.6 \\
\hline P-101 & Gear Pump & 120 & $\mathbf{1}$ & 5 \\
\hline P-102 & Gear Pump & 220 & 3 & 5 \\
\hline P-103 & Gear Pump & 110 & $\mathbf{1}$ & 4.75 \\
\hline P-104 & Gear Pump & 220 & 3 & 4.75 \\
\hline P-105 & Absorber Pump & 110 & $\mathbf{1}$ & 7.4 \\
\hline HE-102 & Mineral Oil Heat Exchanger & 208 & 3 & 65 \\
\hline HE-103 & Vapor Trap & 110 & $\mathbf{1}$ & 3.5 \\
\hline HE-105 & Vapor Trap & 110 & $\mathbf{1}$ & 3.5 \\
\hline RV-101 & Rotary Evaporation & 220 & 2 & 20 \\
\hline VP-101 & Vacuum Pump & 120 & 1 & 13.4 \\
\hline VP-102 & Vacuum Pump & 120 & $\mathbf{1}$ & 21.5 \\
\hline DO-101-3 & Drying Oven & 110 & 1 & 12.5 \\
\hline
\end{tabular}


In addition to the equipment listed above a few additional items might be needed to operate the CEPU. These items include workbench, locked storage locker for tools, fans, a desk, a temperature monitor for the extraction unit, and anything needed for an ordinary laboratory environment.

\section{Parameter Testing Area}

An area may be designated on the Mezzanine as a Parameter Testing Area, which will consist of a table or laboratory bench. The area will house experimental instrumentation to allow for monitoring of the CEPU process at specific intervals. Such tests could include, but not be limited to testing for ash content, coke yield, coke structure, and elemental analyses. In all cases, the instruments or equipment will be bench scaled.

The bench or tabletop will be either a laminate composite or a solid polymer top. The surface of the bench top will be lined with plastic coated absorbent lab bench material to contain spillage.

\section{Entrance and Exit}

Entrance and exiting to the Mezzanine area will be through the High Bay. The associated fire door exit on the mezzanine level will only be used for emergency or cooling as required. Users will be responsible for locking and maintaining the security of the High Bay area.

The user may request that a locking gate be placed at the stairway landing located roughly halfway up the stairway to provide some form of security to the area. The gate will be installed at the user's expense.

\section{Laboratory Personnel}

The training needed to be provided to researchers and the determination of protective clothing for researchers are to be determined as stipulated in the Presumptions Section, Item Number 5. The agreed-to training and protective apparel are attached to this document as an addendum (page 13). The following remarks are provided as guidance and were taken from an EPA procedure in dealing with hazardous materials.

\section{Addendum}




\section{Training and Personal Protective Equipment}

\section{Training}

- Two trained operators must be present on the Mezzanine before operation of the CEPU

- Operators are to know:

- how to operate each and every piece of equipment;

- where the fire extinguisher and emergency spill kits are located, and be trained in how to use them;

- exactly who to call and what to do in the case or a emergency such as a fire, spill, or injury;

- the material(s) safety properties without looking at the MSDS sheets;

- where the MSDS sheets are located; and

- the materials used and stream properties before and after each piece of equipment;

\section{Personal Protective Equipment}

Personnel must wear:

- safety glasses or face shields at all times;

- chemically resistant clothing when handling chemicals;

- lab coats or coveralls at all times;

- dust masks when dealing with fine dry materials; and

- solid toed leather shoes - no sandels! 


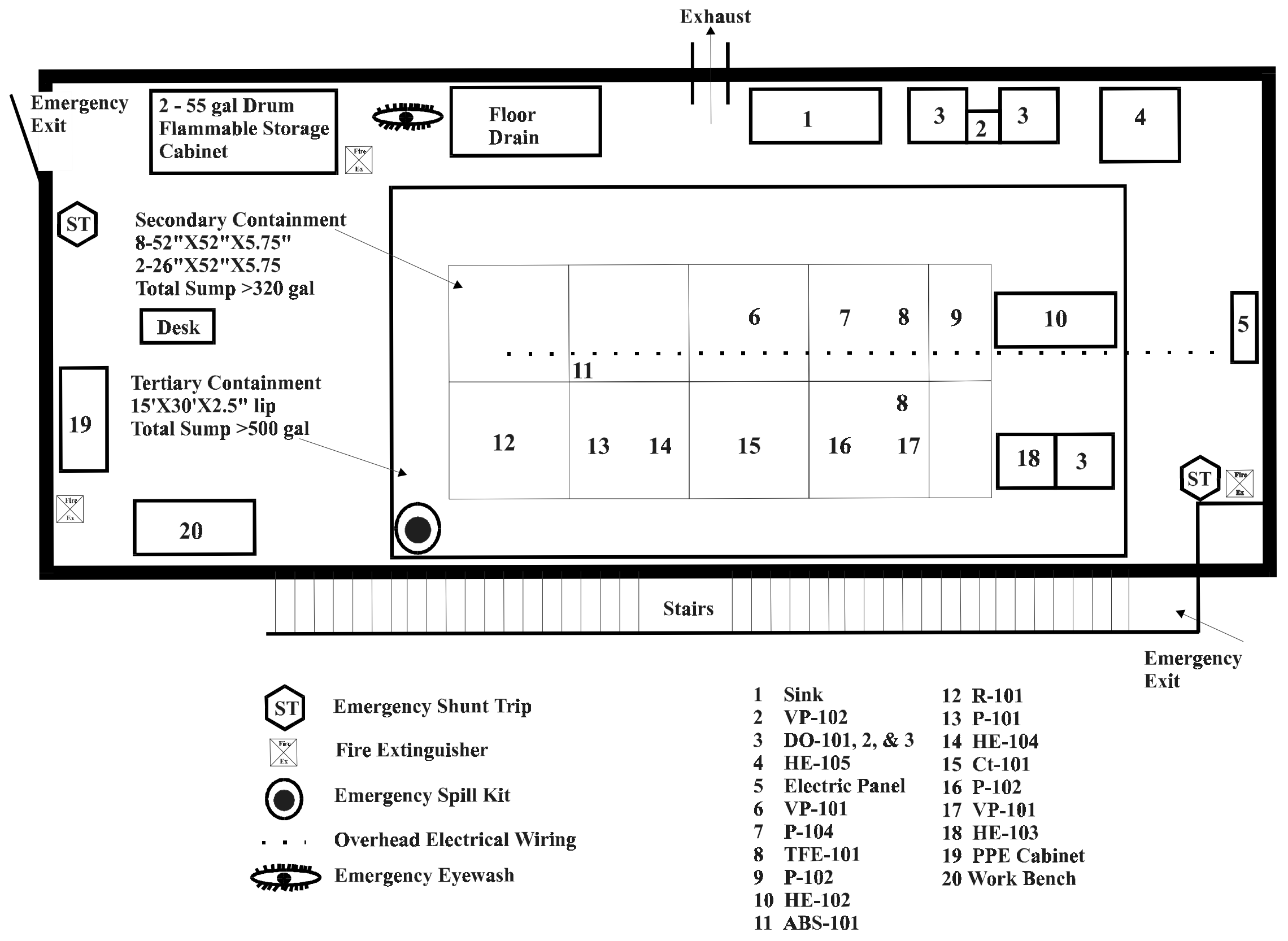

Figure A-1 CEPU Layout in NRCCE High Bay Mezzanine 


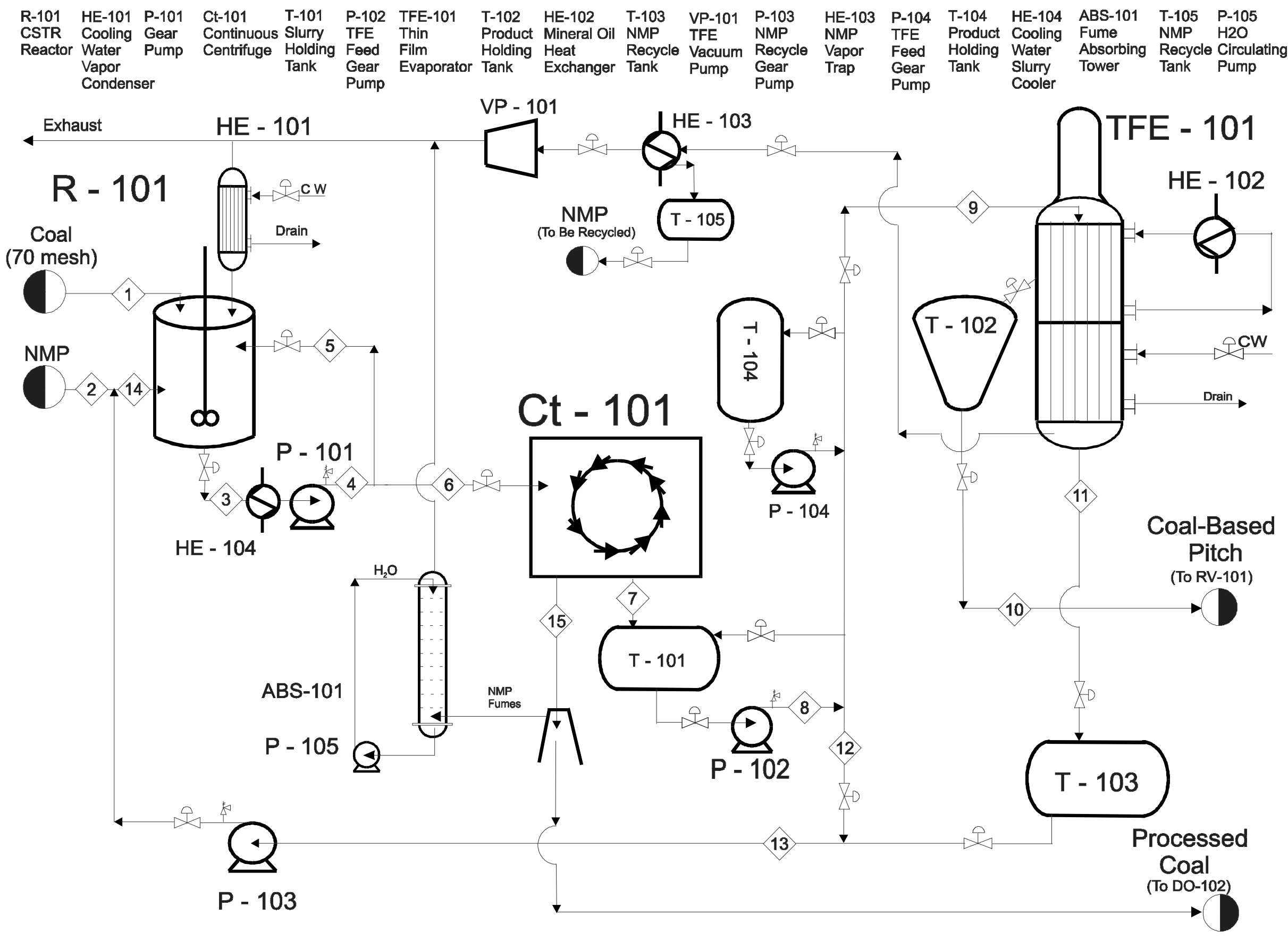

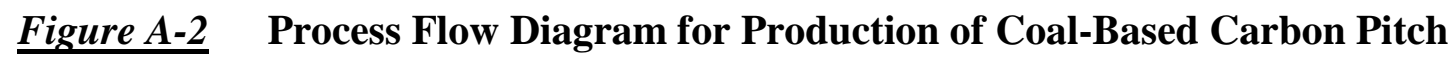




\begin{tabular}{|c|c|c|c|c|c|}
\hline $\begin{array}{l}\text { RV-101 } \\
\text { Roto-Vap }\end{array}$ & $\begin{array}{l}\text { DO-101 } \\
\text { Pitch } \\
\text { Drying } \\
\text { Oven }\end{array}$ & $\begin{array}{l}\text { DO-102 } \\
\text { Waste Coal } \\
\text { Drying Oven }\end{array}$ & $\begin{array}{l}\text { DO-103 } \\
\text { Waste Coal } \\
\text { Drying Oven }\end{array}$ & $\begin{array}{l}\text { HE-105 } \\
\text { Vapor } \\
\text { Trap }\end{array}$ & $\begin{array}{l}\text { T-106 } \\
\text { NMP } \\
\text { Recycle } \\
\text { Tank }\end{array}$ \\
\hline
\end{tabular}

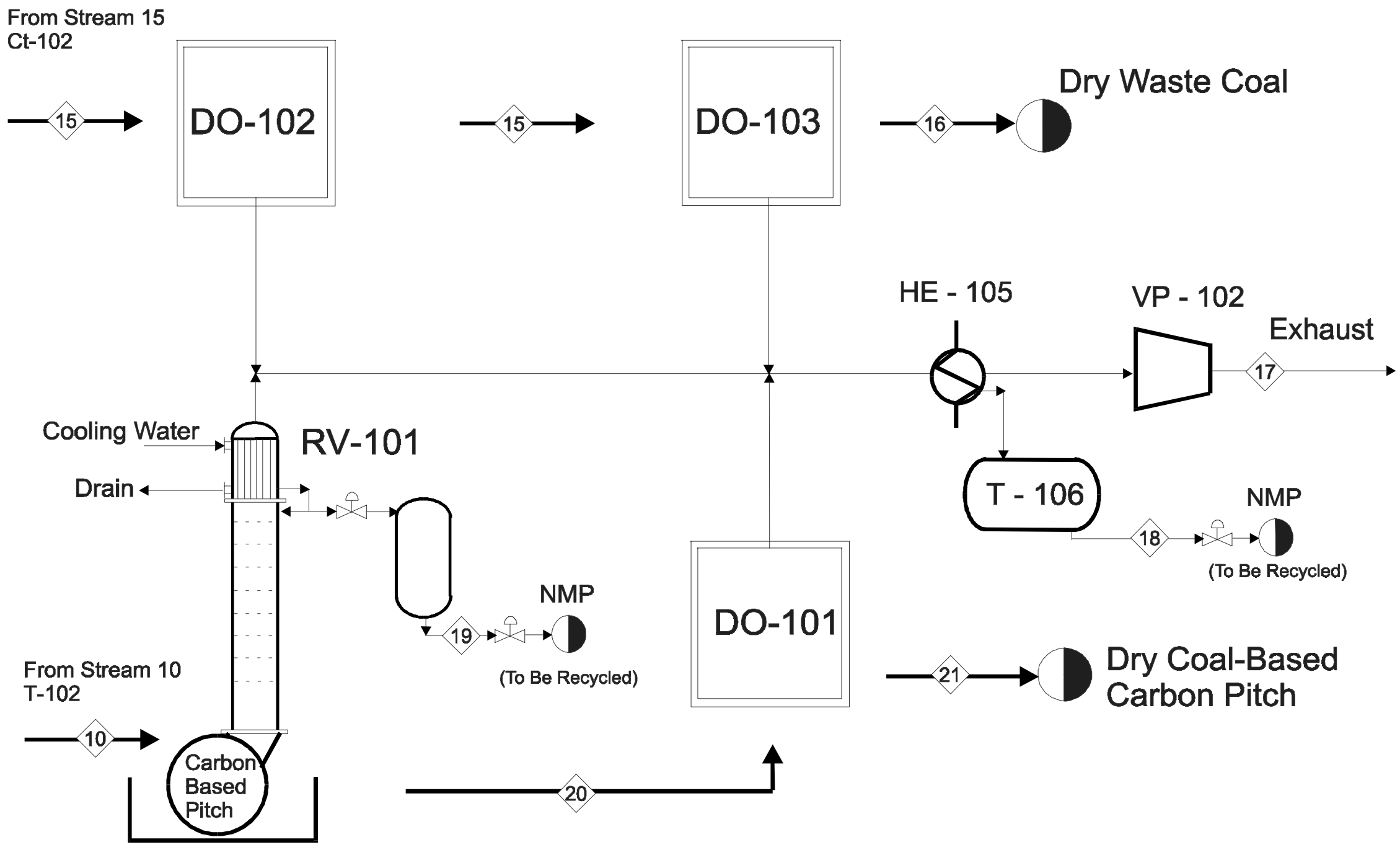




\section{$\underline{\text { Table A-2 }}$ Coal Extraction Pilot Unit Stream Table}

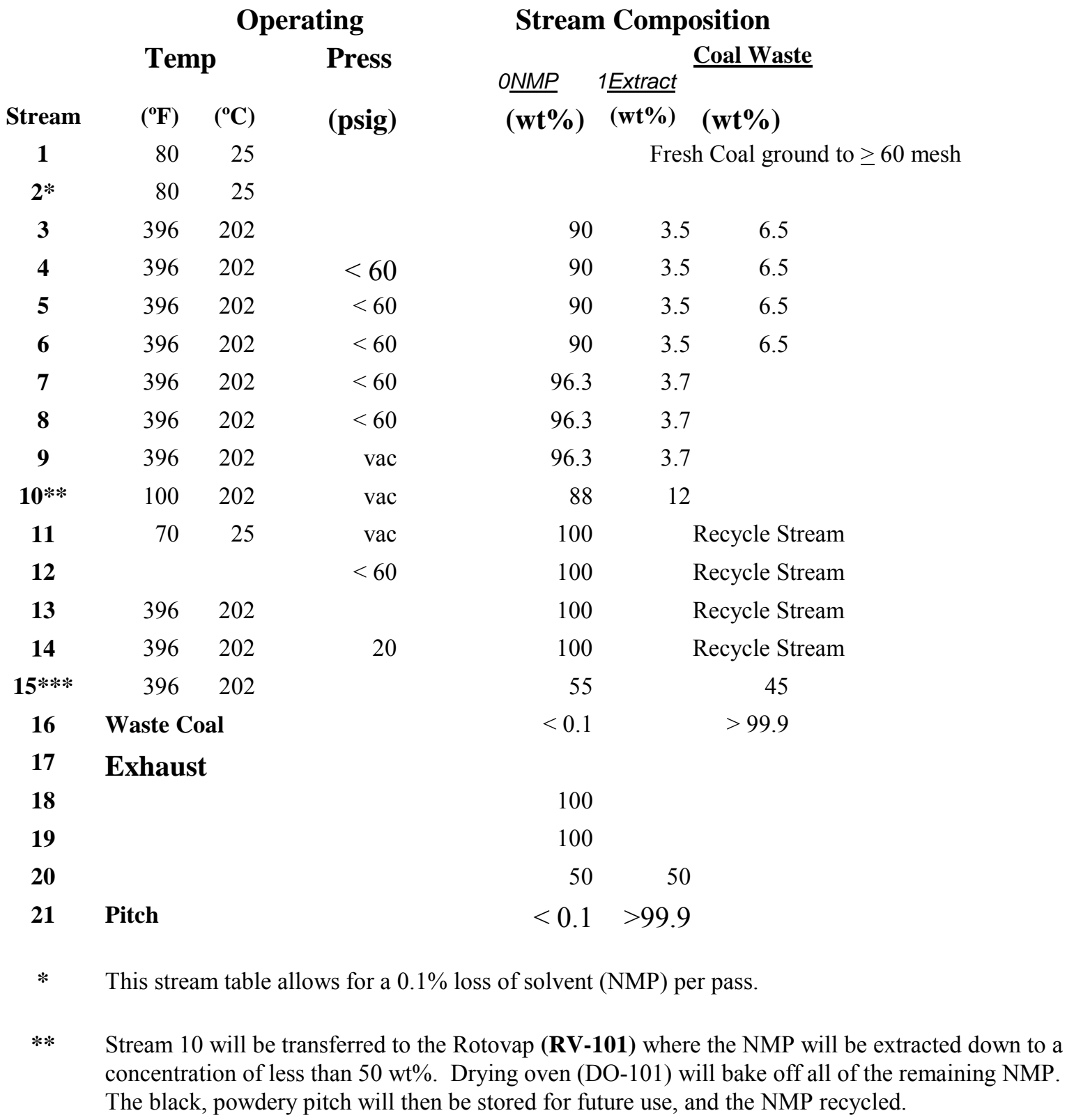

*** Stream 15 will be transferred to drying ovens (DO-102 \& 103) where the remaining NMP will be extracted down to a concentration of less than $1 \mathrm{ppm}$ NMP. The processed coal will then be in powdered form again. The only difference is the used coal will have a higher ash (mineral) content.

No clean up process shown on PFD for the $\mathrm{H}_{2} \mathrm{O} / \mathrm{NMP}$ condensed by the reactor condenser (HE-101). 


\section{Table A-3 Equipment List for Coal Extraction Pilot Unit}

\section{Equipment List}

\# Discription

CSTR Reactor (with Stirrer \& Heater)

Ct-101 Sharples Penwalt Centrifuge

TFE-101 Pfaudler Wiped Thin Film Evaporator

RV-101 Buchi R-135 Rotary Evaporator

DO-101 NAPCO Model 5961Vacuum Drying Oven

DO-102 NAPCO Model 5961Vacuum Drying Oven

DO-103 NAPCO Model 5961Vacuum Drying Oven

ABS-101 NMP Vapor Absorbing Tower

HE-101 Reactor NMP/ $\mathrm{H}_{2} \mathrm{O}$ Vapor Condenser

HE-102 Heater for TFE (Mineral Oil)

HE-103 Vapor Trap/Condenser for VP-101

HE-104 Reactor Slurry Cooler before Centrifuge

HE-105 Drying Oven Condenser \& VP-102 Vapor Trap

P-101 Tuthill Gear Pump Model 25A D.I. 0.5

P-102 Tuthill Gear Pump Model 5A D.I.

P-103 Magnetic Drive NMP Recycle Pump $\quad 0.125$

P-104 Tuthill Gear Pump Model 25A D.I.

P-105 Absorber $\mathrm{H}_{2} \mathrm{O}$ Recycling Pump (Centrifugal) 0.5

VP-101 Vacuum Pump for TFE

VP-102 Vacuum Pump for Drying Ovens \& Rotovap 1

T-101 Centrifuge Product Tank NMP/Disssolve Coal)

T-102 TFE Product Tank (NMP/Dissolved Coal)

T-103 Tank for Condensed NMP from TFE

T-104 Upright Measuring Tank

T-105 Tank for VP-101 Condenser / Vapor Trap

T-106 Condenser Tank for Vapor Trap for Drying Oven

\begin{tabular}{ccccr} 
& & & & \\
hp) & (kW) & (Volts) & (Amps) & (Ph \\
\hline 1 & 13 & 220 & 60 & 1 \\
7.5 & & 220 & 21 & 3 \\
3 & & 220 & 7.6 & 3 \\
& 3.2 & 220 & 145 & 3 \\
& 1.5 & 120 & 12.5 & 1 \\
& 1.5 & 120 & 12.5 & 1 \\
1.5 & 120 & 12.5 & 1
\end{tabular}

\section{Vol}

(gal)

3

$\begin{array}{ccc}220 & 65 & 3 \\ 110 & 5 & 1\end{array}$

5

$\begin{array}{lll}110 & 5 & 1 \\ 110 & & 1\end{array}$

\section{0}

220 6.2

220

220

110

110

110

7.4

11

60

30

30
60

60

5

20
Surface Area Oper. Press Oper. Temp

Inside|Outside Max $\mid$ Norm

$\left(\mathbf{f t}^{2}\right)$

$\left(\mathrm{ft}^{2}\right)$

(psia)

\begin{tabular}{lc} 
(psia) \\
\hline 9.6 & 14.6
\end{tabular}

1.2

1.3
6.5

(14.

$295<0.2-650-408$

$14.6<0.2 \quad 356 \quad 230$

$14.6<0.2 \quad 500 \quad 410$

$14.6<0.2 \quad 500 \quad 410$

$14.6<0.2 \quad 500 \quad 410$

$14.6 \quad 14.6$

80

$\begin{array}{cc}500 \quad 396 \\ 600 & 408\end{array}$

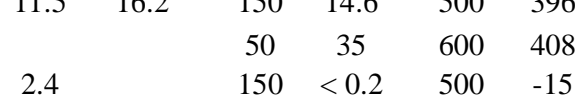

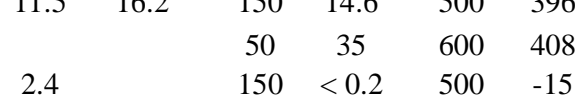

Flow Rate Min $\overline{\text { Max }}$ Norm $(\mathrm{gal} / \mathrm{min})(\mathrm{gal} / \mathrm{min})(\mathrm{gal} / \mathrm{min})$

$\begin{array}{lll}0 & 5 & 1\end{array}$

$\begin{array}{lll}0.2 & 2 & 0.5\end{array}$

$5 \quad 36 \quad 15$

$\begin{array}{cccccc}7.3 & 7.85 & 60 & 20 & 600 & 396 \\ 15.5 & 19.6 & 75 & <0.2 & 400 & -15\end{array}$

$200 \quad 20 \quad 525 \quad 396$

$200 \quad 20 \quad 525 \quad 200$

$25 \quad 5$

$200 \quad 20$

$40 \quad 30$

$14.6<0.2$

$14.6<0.2$

$120 \quad 14.6$

$120<0.2$

$120<0.2$

$120 \quad 14.6$

$120<0.2$

$120<0.2$ 


\section{1-METHYL-2-PYRROLIDINONE}

MSDS Number: M7114 --- Effective Date: 11/17/99

\section{Product Identification}

Synonyms: N-methylpyrrolidinone; N-methyl-2-pyrrolidone; NMP; M-Pyrol

CAS No.: 872-50-4

Molecular Weight: 99.13

Chemical Formula: C5H9NO

Product Codes:

J.T. Baker: 6337, 6347, 6397, 639R, 6407, 9261, R053, R056

Mallinckrodt: 2575, 6392

\section{Composition/Information on Ingredients}

\begin{tabular}{lccl} 
Ingredient & CAS No & Percent & Hazardous \\
\hdashline 1-Methyl-2-pyrrolidinone & $872-50-4$ & $99-100 \%$ & Yes
\end{tabular}

\section{Hazards Identification}

Emergency Overview

WARNING! CAUSES IRRITATION TO SKIN, EYES AND RESPIRATORY

TRACT. HARMFUL IF SWALLOWED OR INHALED.

COMBUSTIBLE LIQUID AND VAPOR.

J.T. Baker SAF-T-DATA(tm) Ratings (Provided here for your convenience)

Health Rating: 1 - Slight

Flammability Rating: 2 - Moderate

Reactivity Rating: 1 - Slight

Contact Rating: 2 - Moderate

Lab Protective Equip: GOGGLES; LAB COAT; CLASS B EXTINGUISHER

Storage Color Code: Red (Flammable)

\section{Potential Health Effects}

\section{Inhalation:}

Mild irritant if vapor or mist from heated solvent is inhaled. Coughing, possible breathing difficulties may be observed.

Ingestion:

Causes irritation to the gastrointestinal tract. Symptoms may include nausea, vomiting and diarrhea.

Skin Contact: 
Mild irritant, may cause some discomfort if in contact with the skin for several hours.

Eye Contact:

Chronic Exposure:

Causes irritation, redness, and pain. May possibly cause corneal clouding.

Minor skin irritation on repeated contact.

Aggravation of Pre-existing Conditions:

No information found.

\section{First Aid Measures}

Inhalation:

Remove to fresh air. If not breathing, give artificial respiration. If Ingestion:

breathing is difficult, give oxygen. Get medical attention.

Induce vomiting immediately as directed by medical personnel. Never give anything by mouth to an unconscious person. Get medical attention.

Skin Contact:

Immediately flush skin with plenty of water for at least 15 minutes. Remove contaminated clothing and shoes. Get medical attention. Wash clothing before reuse. Thoroughly clean shoes before reuse.

Eye Contact:

Immediately flush eyes with plenty of water for at least 15 minutes, lifting lower and upper eyelids occasionally. Get medical attention immediately.

\section{Fire Fighting Measures}

Fire:

Flash point: 93C (199F) CC

Auto-ignition temperature: $346 \mathrm{C}(655 \mathrm{~F})$

Flammable limits in air $\%$ by volume:

Lower: 0.99; Upper: 3.9

Explosion:

Above the flash point, explosive vapor-air mixtures may be formed.

Fire Extinguishing Media:

Dry chemical, foam or carbon dioxide.

Special Information:

In the event of a fire, wear full protective clothing and NIOSH-approved self-contained breathing apparatus with full-face piece operated in the pressure demand or other positive pressure mode.

\section{Accidental Release Measures}

Ventilate area of leak or spill. Remove all sources of ignition. Wear appropriate personal protective equipment as specified in Section 8. Isolate hazard area. Keep unnecessary and unprotected personnel from entering. Contain and recover liquid when possible. Use non-sparking tools and equipment. Collect liquid in an appropriate container or absorb with an inert material (e. g., vermiculite, dry sand, earth), and place in a chemical waste container.

Do not use combustible materials, such as sawdust. Do not flush to sewer! 
J. T. Baker SOLUSORB(R) solvent adsorbent is recommended for spills of this product.

\section{Handling and Storage}

Keep in a tightly closed container. Store in a cool, dry, ventilated area away from sources of heat or ignition. Protect against physical damage. Store separately from reactive or combustible materials, and out of direct sunlight. Containers of this material may be hazardous when empty since they retain product residues (vapors, liquid); observe all warnings and precautions listed for the product.

\section{Exposure Controls/Personal Protection}

\section{Airborne Exposure Limits: \\ AIHA Workplace Environmental Exposure Level (WEEL): 10 ppm, 8-hr, TWA Ventilation System:}

A system of local and/or general exhaust is recommended to keep employee exposures below the Airborne Exposure Limits. Local exhaust ventilation is generally preferred because it can control the emissions of the contaminant at its source preventing dispersion of it into the general work area. Please refer to the ACGIH document, Industrial Ventilation, A Manual of Recommended Practices, most recent edition, for details.

\section{Personal Respirators (NIOSH Approved):}

For conditions of use where exposure to the substance is apparent, consult an industrial hygienist. For emergencies, or instances where the exposure levels are not known, use a full-face piece positivepressure, air-supplied respirator.

WARNING: Air purifying respirators do not protect workers in oxygen-deficient atmospheres.

\section{Skin Protection:}

Wear impervious protective clothing, including boots, gloves, lab coat, apron or coveralls, as appropriate, to prevent skin contact.

\section{Eye Protection:}

Use chemical safety goggles and/or a full face shield where splashing is possible. Maintain eye wash fountain and quick-drench facilities in work area.

\section{Physical and Chemical Properties}

Appearance:

Odor:

Clear, colorless liquid.

Slight amine odor.

Solubility:

Miscible in water.

Specific Gravity:

1.03

pH:

7.7

\% Volatiles by volume @ 21C (70F):

100

Boiling Point: 
202C (396F)

Melting Point:

$-24 \mathrm{C}(-11 \mathrm{~F})$

Vapor Density (Air=1):

3.4

Vapor Pressure (mm Hg):

$0.5 @ 25 \mathrm{C}(77 \mathrm{~F})$

Evaporation Rate $(\mathrm{BuAc}=1)$ :

0.06

\section{Stability and Reactivity}

Stability:

Stable under ordinary conditions of use and storage. Hygroscopic and basic.

Hazardous Decomposition Products:

Burning may produce carbon monoxide, carbon dioxide, and nitrogen oxides.

Hazardous Polymerization:

Will not occur.

Incompatibilities:

Strong oxidants and acids. Reacts with chlorinating agents to form the amide. Reacts with sulfur or carbon disulfide at high temperatures and pressures.

Conditions to Avoid:

Heat, flames, ignition sources and incompatibles.

\section{Toxicological Information}

1-Methyl-2-pyrrolidinone:

Oral rat LD50: $3914 \mathrm{mg} / \mathrm{kg}$;

Skin rabbit LD50: $8 \mathrm{gm} / \mathrm{kg}$;

Investigated as a mutagen, reproductive effector.

\begin{tabular}{|c|c|c|c|c|}
\hline \multirow[b]{2}{*}{ Ingredient } & \multicolumn{3}{|c|}{---NTP Carcinogen--- } & \multirow[b]{2}{*}{ IARC Category } \\
\hline & & Known & Anticipated & \\
\hline 1-Methyl-2 & ne (872-50-4) & No & No & ne \\
\hline
\end{tabular}

\section{Ecological Information}

\section{Environmental Fate:}

When released into the soil, this material may biodegrade to a moderate extent. When released into the soil, this material may evaporate to a moderate extent. When released into water, this material is not expected to evaporate significantly. This material is not expected to significantly bioaccumulation.

When released into the air, this material is expected to be readily degraded by reaction with photo chemically produced hydroxyl radicals. When released into the air, this material may be removed from the atmosphere to a moderate extent by wet deposition. Environmental Toxicity:

The LC50/96-hour values for fish are over $100 \mathrm{mg} / \mathrm{l}$. 


\section{Disposal Considerations}

Whatever cannot be saved for recovery or recycling should be managed in an appropriate and approved waste disposal facility. Processing, use or contamination of this product may change the waste management options. State and local disposal regulations may differ from federal disposal regulations. Dispose of container and unused contents in accordance with federal, state and local requirements.

14. Transport Information

Domestic (Land, D.O.T.)

Proper Shipping Name: COMBUSTIBLE LIQUID, N.O.S. (1-METHYL-2-PYRROLIDINONE)

Hazard Class: COMB

UN/NA: NA1993

Packing Group: III

Information reported for product/size: 460LB

\section{Regulatory Information}

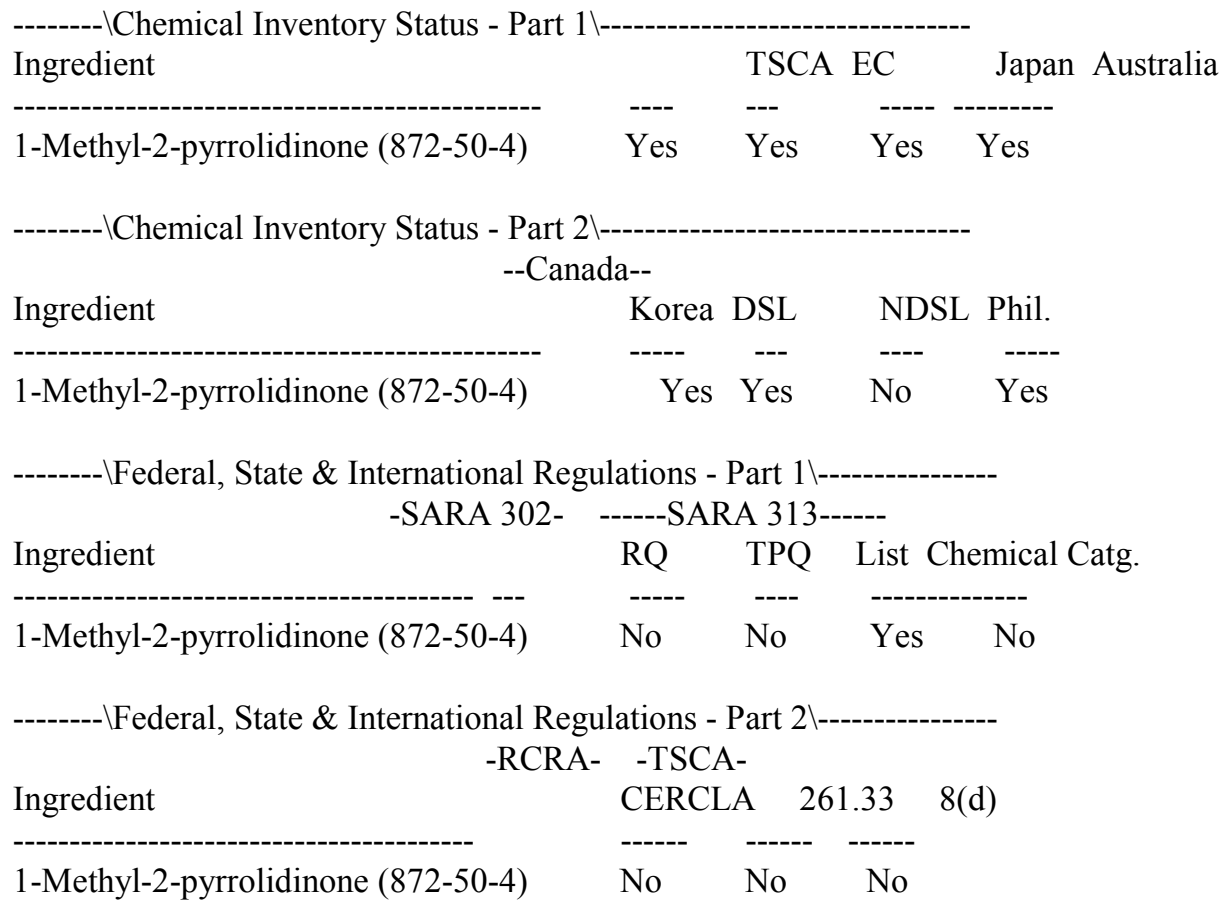

Chemical Weapons Convention: No TSCA 12(b): Yes CDTA: No 
SARA 311/312: Acute: Yes

Chronic: No Fire: No Pressure: No

Reactivity: No (Pure / Liquid)

Australian Hazchem Code: No information found.

Poison Schedule: No information found.

WHMIS:

This MSDS has been prepared according to the hazard criteria of the Controlled Products Regulations

(CPR) and the MSDS contains all of the information required by the CPR.

\section{Other Information}

NFPA Ratings: Health: 2 Flammability: 1 Reactivity: 0

Label Hazard Warning:

WARNING! CAUSES IRRITATION TO SKIN, EYES AND RESPIRATORY TRACT. HARMFUL IF SWALLOWED OR INHALED. COMBUSTIBLE LIQUID AND VAPOR.

Label Precautions:

Avoid contact with eyes, skin and clothing.

Avoid breathing vapor or mist.

Keep container closed.

Use only with adequate ventilation.

Keep away from heat and flame.

Wash thoroughly after handling.

Label First Aid:

In case of contact, immediately flush eyes or skin with plenty of water for at least 15 minutes. Remove contaminated clothing and shoes. Wash clothing before reuse. If inhaled, remove to fresh air. If not breathing, give artificial respiration. If breathing is difficult, give oxygen. If swallowed, induce vomiting immediately as directed by medical personnel. Never give anything by mouth to an unconscious person. In all cases, get medical attention.

Product Use:

Laboratory Reagent.

Revision Information:

No changes.

Disclaimer:

Mallinckrodt Baker, Inc. provides the information contained herein in good faith but makes no representation as to its comprehensiveness or accuracy. This document is intended only as a guide to the appropriate precautionary handling of the material by a properly trained person using this product. Individuals receiving the information must exercise their independent judgment in determining its appropriateness for a particular purpose.

MALLINCKRODT BAKER, INC. MAKES NO REPRESENTATIONS OR WARRANTIES, EITHER EXPRESS OR IMPLIED, INCLUDING WITHOUT LIMITATION ANY WARRANTIES OF MERCHANTABILITY, FITNESS FOR A PARTICULAR PURPOSE WITH RESPECT TO THE INFORMATION SET FORTH HEREIN OR THE PRODUCT TO WHICH THE INFORMATION REFERS. ACCORDINGLY, MALLINCKRODT BAKER, INC. WILL NOT BE RESPONSIBLE FOR DAMAGES RESULTING FROM USE OF OR RELIANCE UPON THIS INFORMATION.

Prepared by: Strategic Services Division

Phone Number: (314) 539-1600 (U.S.A.) 
$\underline{\text { APPENDIX B }}$ 


\section{$\underline{\text { Table B-1 }}$ Key Physical and Chemical Properties of N-Methyl-2-Pyrrolidone}

Structure:<smiles>CN1CCCC1=O</smiles>

Empirical Formula:

Molecular Weight:

Physical form:

Color (APHA):

Melting Point:

Boiling Point:

Viscosity $\left(25^{\circ} \mathrm{C}\right)$

Specific Gravity:

Specific Heat $(\mathrm{Cp})$ :
$\mathrm{C}_{5} \mathrm{H}_{9} \mathrm{NO}$

99.13

Liquid with mild amine-like odor.

50

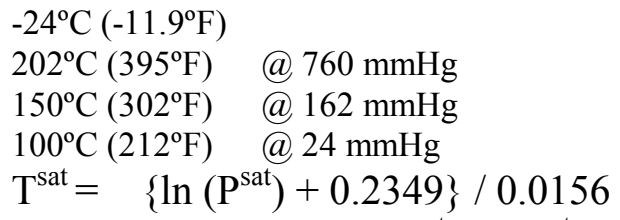

$1.65 \mathrm{cp}$

$1.027 @ 25^{\circ} \mathrm{C}$

$.987 @ 75^{\circ} \mathrm{C}$

$.969 @ 100^{\circ} \mathrm{C}$

$0.40 \mathrm{Kcal} / \mathrm{kg}$ at $20^{\circ} \mathrm{C}$

$$
\mathrm{Cp}_{\mathrm{NMP}}=8.04 * 10^{\wedge}-4 *(\mathrm{~T})+.38
$$

$\left(\mathrm{Cp}\left[\mathrm{cal} / \mathrm{gm}^{* \circ} \mathrm{C}\right], \mathrm{T}\left[{ }^{\circ} \mathrm{C}\right]\right)$

Thermal Conductivity $\left(\mathrm{k}_{\mathrm{NMP}}\right): \quad \mathrm{k}_{\mathrm{NMP}}=-1^{*} 10^{\wedge}-4(\mathrm{~T})+0.1954$ (B-3)

$\left(\mathrm{T}\left[{ }^{\circ} \mathrm{C}\right], \mathrm{k}_{\mathrm{NMP}}\left[\mathrm{W} / \mathrm{M} /{ }^{\circ} \mathrm{C}\right]\right)$

Heat of Vaporization Interfacial Surface Tension $\left(25^{\circ} \mathrm{C}\right): 40.7$ dynes $/ \mathrm{cm}$

Flash Point (open cup):

Dipole Moment

$95^{\circ} \mathrm{C}\left(204^{\circ} \mathrm{F}\right)$

Dielectric Constant $\left(25^{\circ} \mathrm{C}\right)$ :

$4.09+0.04$ Debye

Solubility parameter $(\delta)$ :

Miscibility with Other Solvents:

11.0

completely miscible with water and most organic solvents including alcohols, esters, ketones, aromatic and chlorinated hydrocarbons and vegetable oil. 


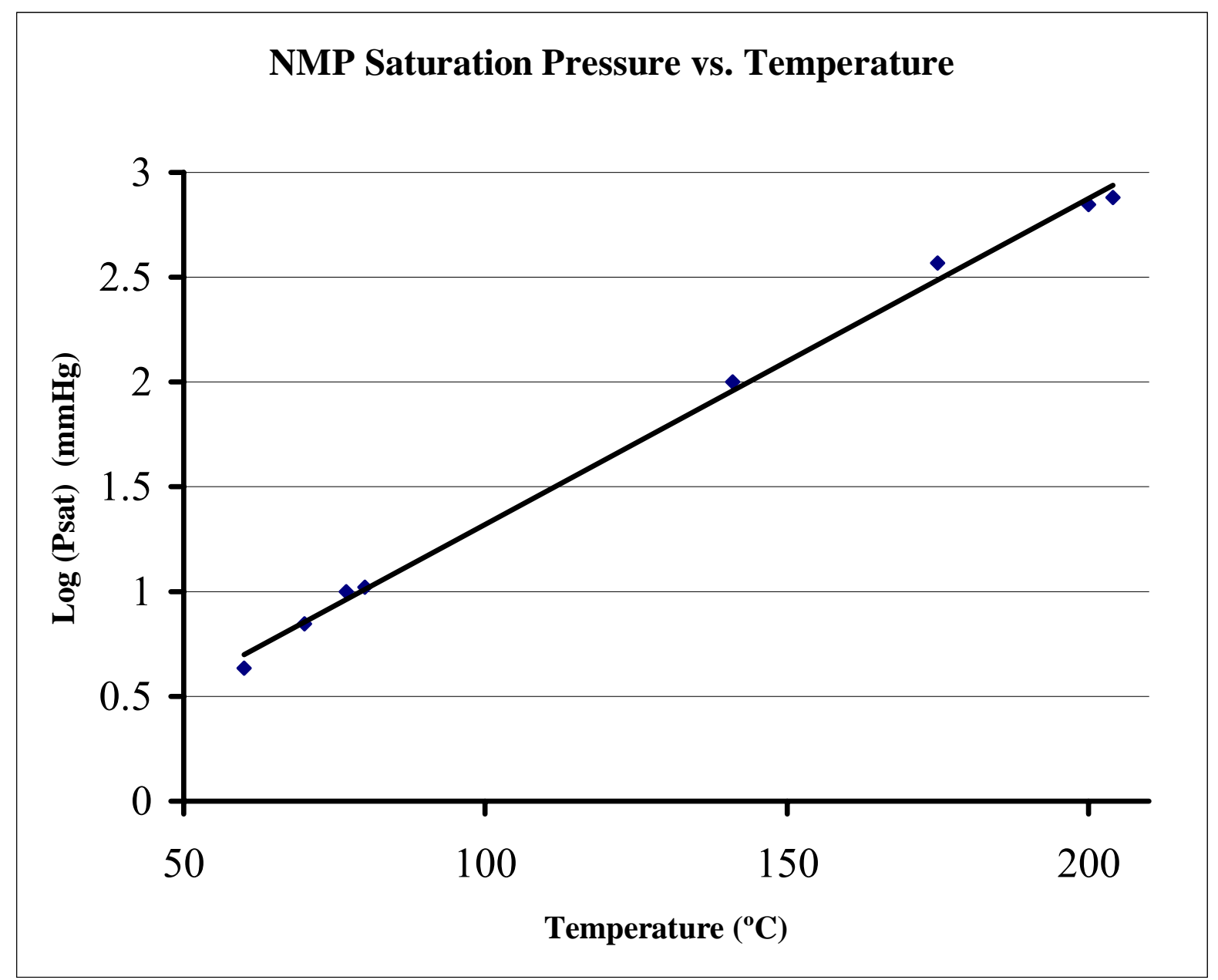

Figure B-1 Log of NMP Saturation Pressure vs. Temperature

From Figure B-1 we can regress the data to form the following equation for the saturation temperature $\left(\mathrm{T}^{\mathrm{sat}}\right)$.

$\mathrm{T}^{\mathrm{sat}}=\left\{\log \left(\mathrm{P}^{\mathrm{sat}}\right)+0.2349\right\} / 0.0156$

where: $\mathrm{T}^{\mathrm{sat}}\left[{ }^{\circ} \mathrm{C}\right]$ is the temperature

$\mathrm{P}^{\mathrm{sat}}[\mathrm{mmHg}]$ is the pressure 


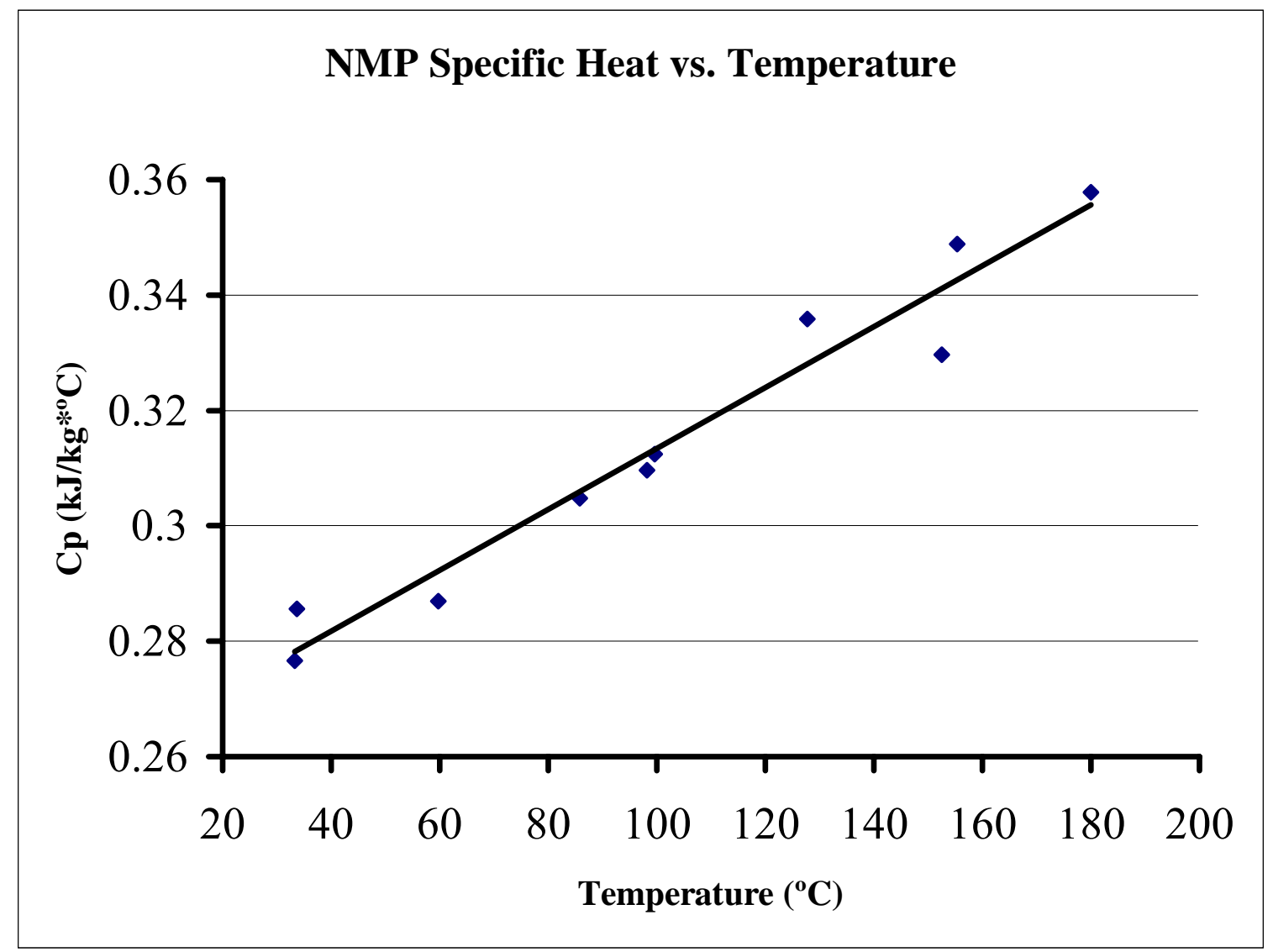

$\underline{\text { Figure B-2 }}$ Specific Heat for NMP Versus Temperature

From Figure B-2 we can regress the data to form an equation for the Specific Heat $\left(\mathrm{C} \mathrm{p}_{\mathrm{NMP}}\right)$.

$\mathrm{Cp}_{\mathrm{NMP}}=3.37 * 10^{\wedge}-3 *(\mathrm{~T})+1.59$

Where: $\mathrm{Cp}\left[\mathrm{cal} / \mathrm{gm}^{* \mathrm{o}} \mathrm{C}\right]$

$\mathrm{T}\left[{ }^{\circ} \mathrm{C}\right]$ 


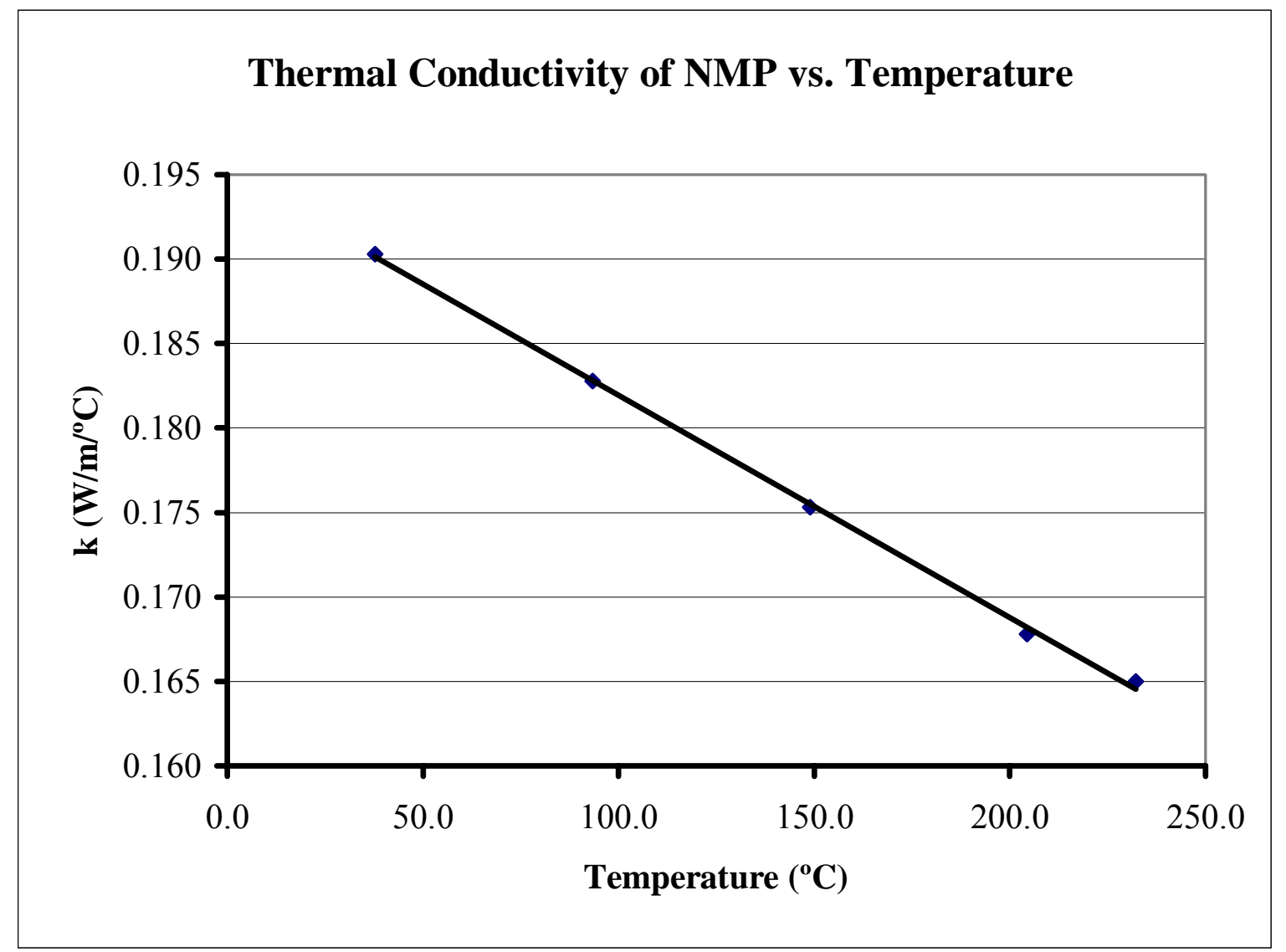

$\underline{\text { Figure B-3 }}$ Thermal Conductivity for NMP Versus Temperature

From Figure \# 3 we can regress the data to form an equation for the thermal conductivity ( $\left.\mathrm{k}_{\mathrm{NMP}}\right)$.

$k_{\mathrm{NMP}}=-1^{*} 10^{\wedge}-4\left(\mathrm{~T}^{\mathrm{sat}}\right)+0.1954$

where: $\quad \mathrm{T}\left[{ }^{\circ} \mathrm{C}\right]$

$\mathrm{k}_{\mathrm{NMP}}\left[\mathrm{W} / \mathrm{M} /{ }^{\circ} \mathrm{C}\right]$ 


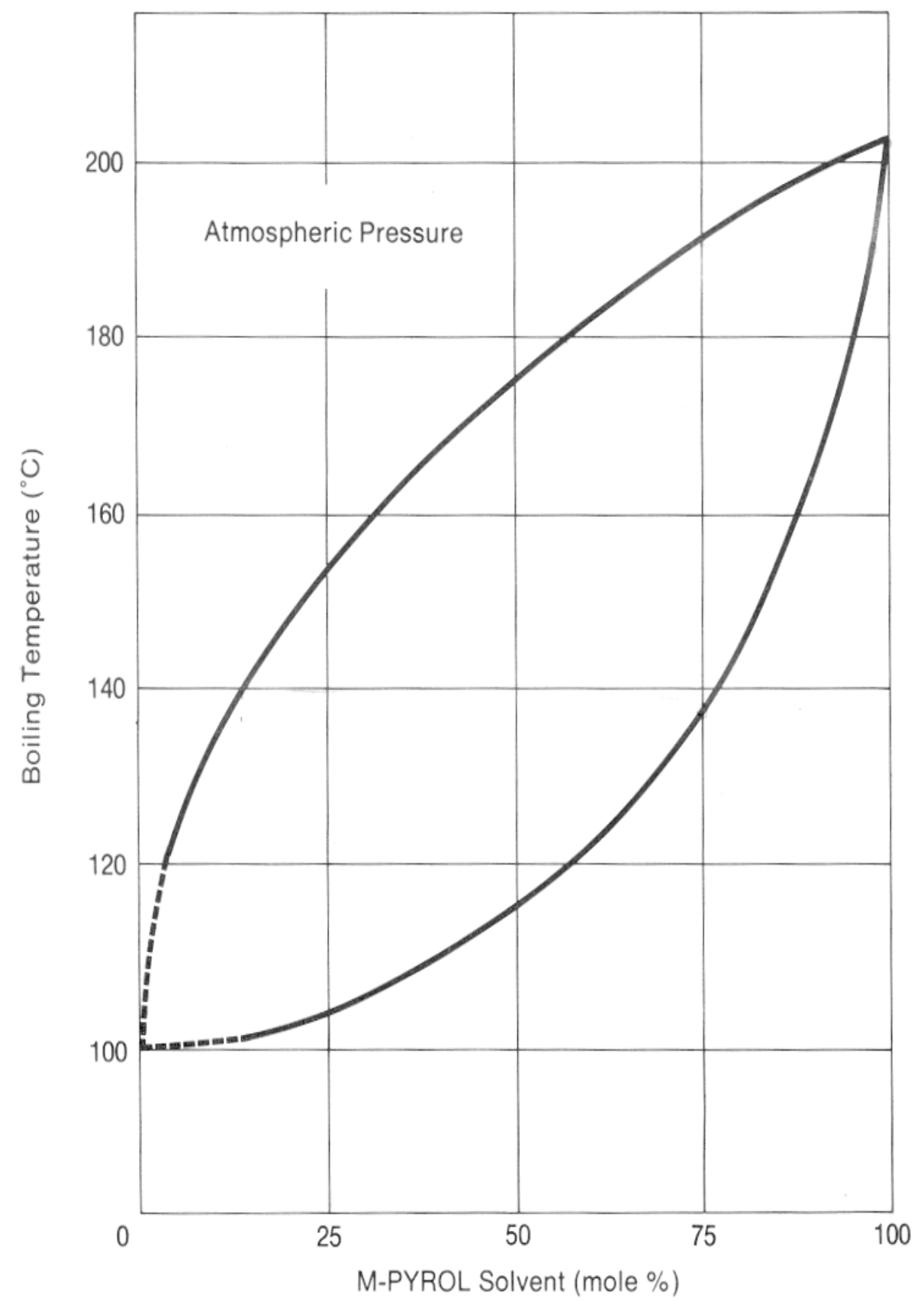

Figure B-4 Vapor/Liquid Equilibrium of NMP Solvent an Water Mixtures

Source: G A F Corporation

Table B-2 Vapor Liquid Equilibrium Data for Water Systems

Based on NMP at Atmospheric and $400 \mathrm{mmHg}$ Pressures 


\section{NMP Solvent}

\begin{tabular}{cccccc}
$\begin{array}{l}\text { OBoiling } \\
\text { Point }\end{array}$ & Pressure & \multicolumn{2}{c}{ (Mole \%) } & \multicolumn{2}{c}{ (Weight \%) } \\
$\left({ }^{\circ} \mathbf{C}\right)$ & $(\mathbf{m m}$ Hg) & In Liquid & In Vapor & In Liquid & In Vapor \\
202 & 757.2 & 99.6 & 95.7 & 99.9 & 99.2 \\
190 & 761.8 & 95.9 & 70.8 & 99.2 & 93.0 \\
186 & 758.2 & 96.7 & 68.6 & 99.4 & 92.3 \\
168 & 400.0 & 97.6 & 77.1 & 99.6 & 94.9 \\
165 & 399.0 & 97.3 & 68.1 & 99.5 & 92.1 \\
162 & 400.4 & 96.6 & 63.8 & 99.4 & 90.6 \\
135 & 757.2 & 72.4 & 10.0 & 93.5 & 37.9 \\
108 & 755.5 & 34.7 & 1.9 & 74.5 & 9.5 \\
102 & 756.0 & 17.4 & 1.0 & 53.7 & 5.3 \\
100 & 756.1 & 2.7 & 0.5 & 13.4 & 2.6
\end{tabular}

\section{OTable B-3 Stability of NMP in Air:}

Air was bubbled through a sample of N-methyl-2-pyrrolidone for 168 hours at $49^{\circ} \mathrm{C}\left(120^{\circ} \mathrm{F}\right)$. Treated and untreated samples were analyzed by VPC with the following results:

N-methyl-2-pyrrolidone

BLO butyrolactone

$\mathrm{N}$-methyl succinimide

Unknown "A"

Unknown "B"

$\begin{array}{cc}\begin{array}{c}\text { Untreated } \\ \text { Sample }\end{array} & \begin{array}{c}\text { Treated } \\ \text { Sample }\end{array} \\ 99.58 \% & 98.86 \% \\ 0.23 \% & 0.24 \% \\ 0.19 \% & 0.84 \% \\ & 0.05 \% \\ & 0.01 \%\end{array}$


APPENDIX C 


\section{Plack Rotk
Test Labs}

WVU Chemical Engineering

PO Box 6102

Morgantown WV 26506

ATTN: Brian Bland

10-11-00

BR\# 37407

Sample ID: 1A fresh ground coal
Telephone (304) 296-8347

5 Eastgate Plaza
- Morgantown, WV 26505

As rcod Dry Dry ash free

PROXIMATE ANALYSIS

$\begin{array}{lrrr}\text { Moisture } & 1.41 \% & ----- & ---- \\ \text { Ash } & 7.32 \% & 7.42 \% & ---- \\ \text { Volatile matter } & 26.45 \% & 26.83 \% & 28.98 \% \\ \text { Fixed carbon } & 64.82 \% & 65.75 \% & 71.02 \%\end{array}$

\section{ULTIMATE ANALYSIS}

Carbon
Hydrogen
Nitrogen
Sulfur
Oxygen
Ash

BTU/1b

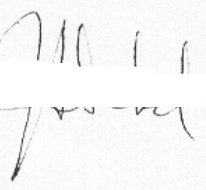

$$
\begin{array}{r}
81.91 \% \\
3.60 \% \\
0.69 \% \\
0.83 \% \\
4.24 \% \\
7.32 \%
\end{array}
$$

14155

$$
\begin{array}{r}
83.08 \% \\
3.65 \% \\
0.70 \% \\
0.84 \% \\
4.31 \% \\
7.42 \%
\end{array}
$$

14357
$89.74 \%$

$3.94 \%$

$0.76 \%$

$0.91 \%$

$4.65 \%$

$-----$

15508 


\section{Deak Doik
Test Lobs}

WVU Chemical Engineering

PO Box 6102

Morgantown WV 26506

ATTN: Brian Bland

10-11-00

BR\#37411

Sample ID: 1B fresh ground coal

\section{MOISTURE}

\section{ULTIMATE ANALYSIS}

Carbon

Hydrogen

Nitrogen

Sulfur

Oxygen

Ash

$\mathrm{BTU} / 1 \mathrm{~b}$

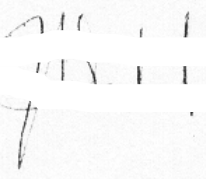

$$
\begin{array}{r}
83.64 \% \\
3.11 \% \\
0.58 \% \\
0.83 \% \\
4.01 \% \\
6.37 \%
\end{array}
$$

14247
Dry

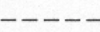

Dry ahs free

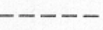

$\begin{array}{rr}84.88 \% & 90.75 \% \\ 3.16 \% & 3.37 \% \\ 0.59 \% & 0.63 \% \\ 0.84 \% & 0.90 \% \\ 4.07 \% & 4.35 \% \\ 6.46 \% & -----\end{array}$

14458

15457

C-2 


\section{$\Leftrightarrow$}

WVU Chemical Engineering

PO Box 6102

Morgantown WV 26506

ATTN: Brian Bland

10-11-00

BR\# 37408

Sample ID: 2A processed coal and NMP

\section{ULTIMATE ANALYSIS}

\begin{abstract}
Carbon
Hydrogen

Nitrogen

Sulfur

Oxygen

Ash
\end{abstract}

As revd Dry

$41.68 \%$
*SEE NOTE*
$* * *$
$2.81 \%$
$0.62 \%$
***
$7.18 \%$

$\mathrm{BTU} / 1 \mathrm{~b}$

12109

NOTE: Moisture due primarily to solvent. Calculations assume moisture determination is due to water. Oxygen value including oxygen in the "moisture" is $16.57 \%$ on the "as rcvd" basis. No correction was made on the as rcvd hydrogen.

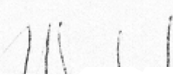




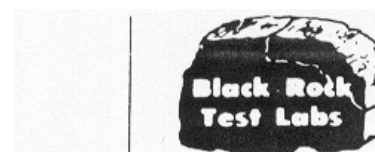

WVU Chemical Engineering

PO Box 6102

Morgantown WV 26506

ATTN: Brian Bland

10-11-00

BR\#37409

Sample ID: 3 A clean coal extract "pitch"
Telephone (304) 296-8347

5 Eastgate Plaza
As revd Dry Dry ash free

PROXIMATE ANALYSIS

Moisture

Ash

$0.71 \%$

Volatile matter

Fixed carbon

$0.64 \%$

$30.34 \%$

$68.31 \%$

$0.64 \%$

$30.56 \%$

$68.80 \%$

$30.76 \%$

$69.24 \%$

\section{ULTIMATE ANALYSIS}
Carbon
Hydrigen
Nitrogen
Sulfur
Oxygen
Ash

$$
\begin{array}{r}
88.32 \% \\
3.63 \% \\
0.55 \% \\
0.70 \% \\
5.45 \% \\
0.64 \%
\end{array}
$$

BTU/1 b
15269

15378
$89.53 \%$

$3.68 \%$

$0.56 \%$

$0.71 \%$

$5.52 \%$

$5.49 \%$

$0.64 \%$ 


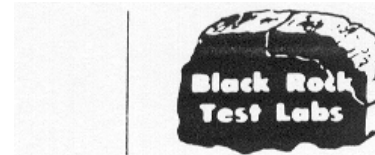

5 Eastgate Plaza

Telephone (304) 296-8347

- Morgantown, WV 26505

WVU Chemical Engineering

PO Box 6102

Morgantown WV 26506

ATTN: Brian Bland

10-11-00

BR\# 37412

Sample ID: 3B clean coal extract "pitch"

\section{MOISTURE}

As revd Dry

$9.21 \%$

$----$

Dry ash free

\section{ULTIMATE ANALYSIS}

Carbon

Hydrogen

Nitrogen

Sulfur

Oxygen

Ash

$$
\begin{gathered}
86.27 \% \\
2.68 \% \\
1.16 \% \\
0.54 \% \\
(-0.41 \%) \\
0.55 \%
\end{gathered}
$$

$$
\begin{gathered}
95.02 \% \\
2.95 \% \\
1.28 \% \\
0.59 \% \\
(-.045 \%) \\
0.61 \%
\end{gathered}
$$

$$
\begin{array}{r}
95.60 \% \\
2.97 \% \\
1.29 \% \\
0.60 \%
\end{array}
$$

$(-0.46 \%)$ SEE NOTE
BTU/1 b
14805
16307
16406

NOTE: Moisture in all probability due to a solvent. Oxygen is derived by difference and the assumption is a portion is contained in the moisture. Oxygen value including oxygen in the "moisture" is $8.80 \%$ and the corresponding hydrogen value is $3.71 \%$, both "as rcvd".

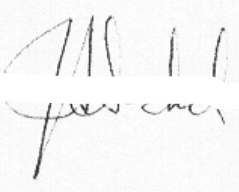




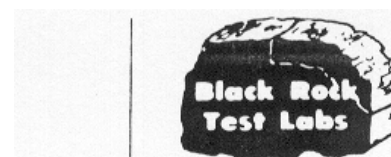

WVU Chemical Engineering

PO Box 6102

Morgantown WV 26506

ATTN: Brian Bland

10-11-00

BR\# 37410

Sample ID: 4 A dried processed coal
Telephone (304) 296-8347

5 Eastgate Plaza

- Morgantown, WV 26505

\section{MOISTURE}

ULTIMATE ANALYSIS

$$
\begin{aligned}
& \text { As rcvd } \\
& 7.69 \% \\
& \\
& 77.19 \% \\
& 2.82 \% \\
& 0.40 \% \\
& 0.70 \% \\
& 2.82 \% \\
& 8.38 \%
\end{aligned}
$$$$
\text { Dry }
$$$$
----
$$

Dry ash free

$\begin{array}{rr}3.62 \% & 91.97 \% \\ 3.05 \% & 3.36 \% \\ 0.43 \% & 0.48 \% \\ 0.76 \% & 0.83 \% \\ 3.06 \% & 3.36 \% \\ 9.08 \% & -----\end{array}$

\section{$\mathrm{BTU} / 1 \mathrm{~b}$}

13197

14296

15724

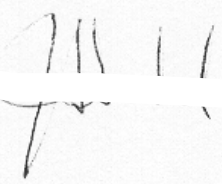




\section{Dlack Dodk
Test Labs}

WVU Chemical Engineering

PO Box 6102

Morgantown WV 26506

ATTN: Brian Bland

\section{Eastgate Plaza \\ Telephone (304) 296-8347 \\ - Morgantown, WV 26505}

$10-11-00$

BR\# 37413

Sample ID: 4B dried processed coal

As revd Dry Dry ash free

MOISTURE

$7.09 \%$

-----

-----

ULTIMATE ANALYSIS

$\begin{array}{lrrr}\text { Carbon } & 77.30 \% & 83.20 \% & 91.79 \% \\ \text { Hydrogen } & 2.59 \% & 2.79 \% & 3.08 \% \\ \text { Nitrogen } & 0.54 \% & 0.58 \% & 0.64 \% \\ \text { Sulfur } & 0.66 \% & 0.71 \% & 0.78 \% \\ \text { Oxygen } & 3.12 \% & 3.36 \% & 3.71 \% \\ \text { Ash } & 8.70 \% & 9.36 \% & -----\end{array}$

BTU/1b

13163

14167

15631

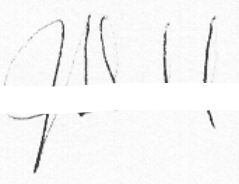




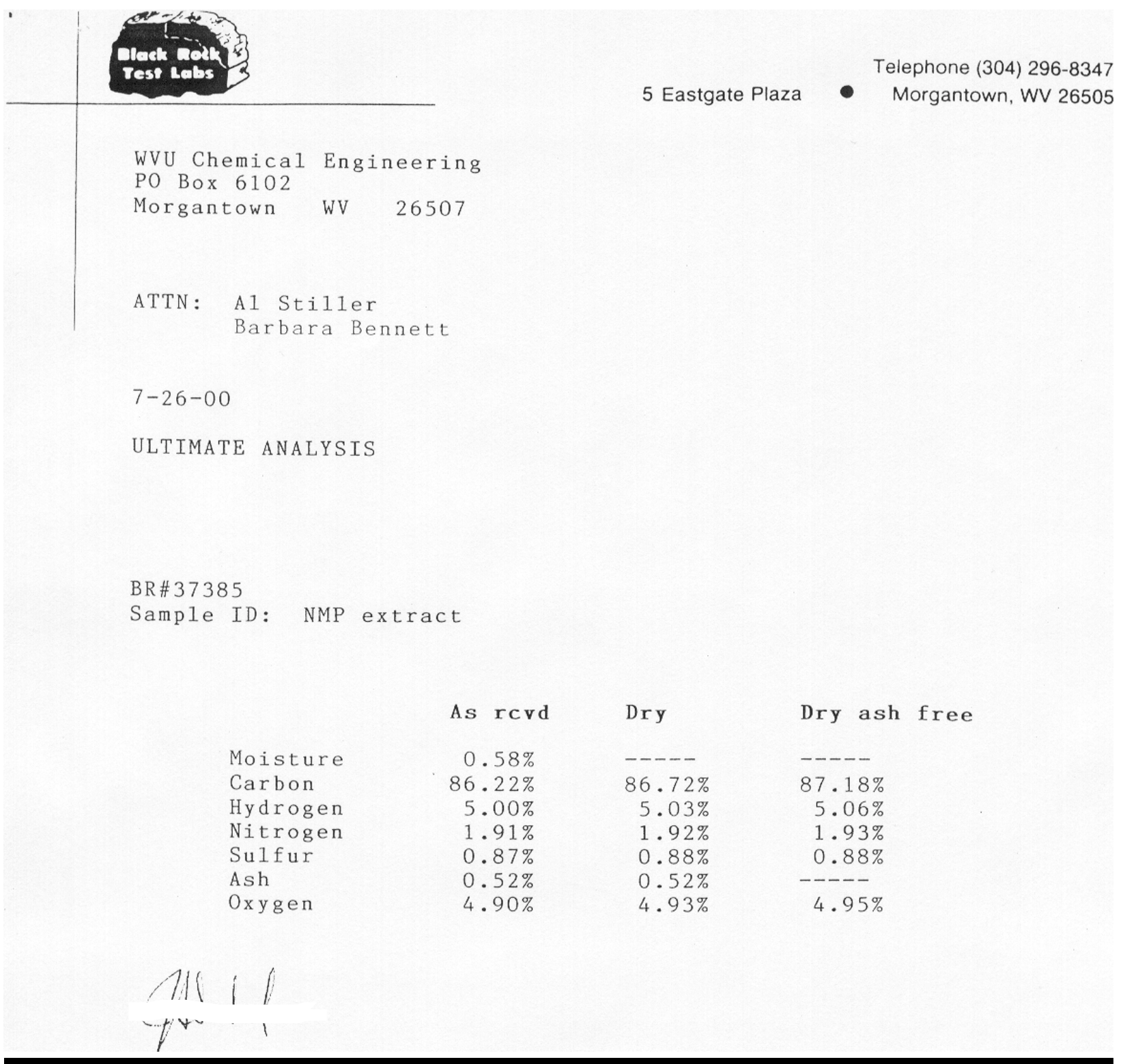




WVU Chemical Engineering
PO Box 6102
Morgantown WV 26507
ATTN: Al Stiller
Barbara Bennett
7-26-00
ULTIMATE ANALYSIS

\section{Eastgate Plaza - Morgantown, WV 26505}

BR\# 37386

Sample ID: NMP extract oxidized at $125^{\circ} \mathrm{C}$ for 2 hours

$\begin{array}{lrrr} & \text { As rcvd } & \text { Dry } & \text { Dry ash free } \\ \text { Moisture } & 0.31 \% & --- & - \\ \text { Carbon } & 6.25 \% & 86.52 \% & 87.27 \% \\ \text { Hydrogen } & 4.92 \% & 4.94 \% & 4.98 \% \\ \text { Nitrogen } & 1.72 \% & 1.73 \% & 1.74 \% \\ \text { Sulfur } & 0.50 \% & 0.50 \% & 0.51 \% \\ \text { Ash } & 0.86 \% & 0.86 \% & ---- \\ \text { Oxygen } & 5.44 \% & 5.45 \% & 5.50 \%\end{array}$

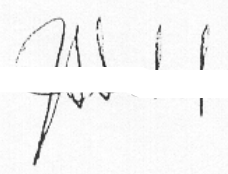




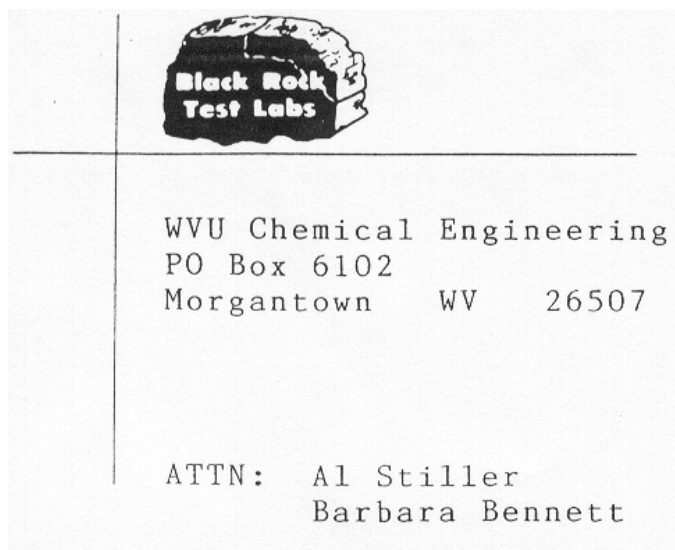

$7-26-00$

ULTIMATE ANALYSIS

BR\# 37387

Sample ID: NMP extract oxidized at $225^{\circ} \mathrm{C}$ for 4 hours
Barbara Bennett
ANALYSIS
5 Eastgate Plaza
Telephone (304) 296-8347
- Morgantown, WV 26505 


\section{APPENDIX D}


Charles Ross \& Son Company

710 Old Willets Path

P.O. Box 12308

Hauppauge, New York 11788-0615

\section{REGIONAL SALES MANAGER}

Jim Van Vranken

Tel: 304-232-5184

Fax: 304-232-5183

\section{CHARLES ROSS \& SON COMPANY}

\section{TEST REPORT}

TODAY'S DATE:

March 14, 2000

TEST CONDUCTED:

March 9, 2000

West Virginia University

Department of Chemical Engineering

413 Engineering Sciences Building

PO Box 6102

Tel: 304-293-2111 X408

Fax: 304-293-4139

WITNESSED TEST CONDUCTED BY CHRIS KUSNIER FOR:

Mr. Brian Bland

MACHINE: HOM-40

MATERIAL: Coal Extract

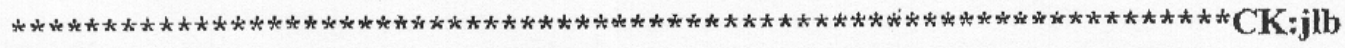

The Department of Chemical Engineering at West Virginia University has recently developed a process to manufacture Carbon Fiber and Fire Resistant Insulation Board derived from Carbon from coal. Crushed coal is dispersed in a low viscosity solvent NMethyl-2-Pyrrolidone. This solvent extracts a certain product from the coal, which is then dried using heat and vacuum to produce a very fine powder similar to graphite. The process currently is being done in the laboratory using rotary evaporators. The test in the HDM-40 starts by charging into the 40 gallon mix can 36 gallons of the coal/NMP dispersion. This is a relatively low viscosity due to the high amount of solvent in the mix. The 40 gallon mix can is heated with the $40 \mathrm{~K}$.W. Chromolox Oil Heating Unit which is preset to $300^{\circ} \mathrm{F}$. The stirrer blades are started at 12 rpm drawing 11 amps on the $15 \mathrm{HP}$ motor. For recovery of the solvent a Helex Shell and Tube Condenser is connected inline between the vacuum

Phone: 631/234-0500 - Fax; 631/234-0691 • e-mail: mail@rossmixing.com • www.rossmixing.com 
purnp and HDM-40. A vacuum is drawn on the product to 27 inches mercury and nitrogen is purged through the system at the opposite side of the vacuum hood. At 20 minutes the stirrer blades speed is increased to $20 \mathrm{rpm}$ drawing 11 amps.

Forty minutes: Product temperature is now $165^{\circ} \mathrm{F}$ we continue mixing.

One hour twenty minutes: Product temperature is $200^{\circ} \mathrm{F}$.

Two hours: We now draw off from the knockout pot in the Helex Condenser 1.5 gallons of NMP solvent. Material temperature is $214^{\circ} \mathrm{F}$.

Three hours: Material temperature is $225^{\circ} \mathrm{F}$ we noticed through the sight ports of the vacuum hood there is a high degree of refluxing occurring due to the high surface area in the vacuum hood and gear box area in the HDM-40.

Four hours: Another 10 gallons of solvent are recovered from the knockout pot and the material temperature is now $240^{\circ} \mathrm{F}$ and at this higher temperature we are able to draw off more solvent. It is necessary to draw off a total of approximately 30 gallons of solvent to thoroughly dry this material.

Five hours: Another 7 gallons of solvent is recovered material temperature is $241^{\circ} \mathrm{F}$. Inside the mix vessel a $60 \%$ reduction in batch volume is noticed. The vacuum is now up to 28 inches mercury continue mixing.

Five hours thirty minutes: More solvent is discharged from the condenser at this point a total of 19 gallons has been recovered, however, the refluxing in the vacuum hood is reducing our rate of solvent recovery. At this point we start cooling the oil for discharge of the material. It is also noticed that there is a slight build up of material on the inner side wall of the mix can indicating that the use of a scraper arm would be beneficial for thermal transfer. To reduce the effects of the refluxing it is recommended that the vacuum hood is jacketed for hot oil or in addition a liquid seal can be utilized.

The material is then be discharged into 5 gallon palls supplied by Mr. Bland and is taken back to the University with him the machine cleans easily with water there is no charge for testing and Jim Van Vranken will follow up with Brain Bland. 
Charles Ross \& Son Company

710 Old Willets Path

P.O. Box 12308

Hauppauge, New York 11788-4193

ROSS

REGIONAL SALES MANAGER

Jim Van Vranken

Fax: (304) 232-5183

\section{CHARLES ROSS \& SON COMPANY}

\section{TEST REPORT}

$\begin{array}{ll}\text { TODAY'S DATE: } & \text { April 27, } 2000 \\ \text { TEST CONDUCTED: } & \text { April 25, 2000 }\end{array}$

University of West Virginia

Dept. of Chemical Engineering

413 Engineering Sciences Bldg.

Morgantown, WV 26506

Tel. $\quad 304-293-2111$ ex. 408

Fax. 304-293-4139

WTTNESSED TEST CONDUCTED BY CHRIS KUSNIER FOR:
Mr. Brian Bland

MACHINE: HDM-40 Double Planetary Mixer

MATERIAL: Coal Extract

$* * * * * *+* * * * * * * * * * * * * * * * * * * * * * * * * * * * * * * * * * * * * * * * * * * * * * * * * * * * * * * * * * * * * * * * * \mathrm{CK}: \mathrm{VC}$

The test today is a continuation of a test from March $9^{\text {th }}$ of this year where Mr. Bland is hoping to vacuum dry coal dispersed in a low viscosity solvent N-Methyl-Pyrrolidone. The coal is dried and then used to process carbon fiber and fire-resistant insulation boards. The test involves the HDM- 40 which is configured with oil heating from the Chromalox $40 \mathrm{kw}$ oil heater. A teflon scraper arm is attached to the fabricated gearbox in the HDM-40. To prevent the condensation and refluxing of the solvent inside the upper vacuum hood area, Mr. Bland has brought a number of electric heating bands which are wrapped around the vacuum hood and fiberglass insulation which is also wrapped over the heating bands. The solvent will be collected through a Helex shell and tube condenser, a $20 \mathrm{cfm} 1 \mathrm{HP}$ vacuum pump in-line with the Helex will draw the solvent off and nitrogen gas is used to strip the vapors.

Phone: 631/234-0500 - Fax: 631/234-0691 - e-mail: mail@rossmixing.com 
Lato the 40-gallon mix can, approximately 30 gallons of the coal/NMP is charged. The mix can is then heated pre-set at 400 degrees $\mathrm{F}$ and the standard rectangular stirrer blades are started at 20 rpm drawing 11 amps on the $15 \mathrm{HP}$ motor, 20 amps is the FLC. Continue to mix at these parameters. A small amount of approximately 1-2 psi of nitrogren is purged through the vacuum hood to aid in the NMP vapors being drawn through the condenser.

2 HOURS The vacuum is broken and one gallon of NMP solvent is drawn from the knockout pot at the base of the condenser. Material temperature is 232 degrees $\mathrm{F}$ and at this point it is noticed that there is heavy condensing inside the vacuum hood.

3 HOURS

Another two gallons of solvent is drawn from the knock-out pot. Material $30 \mathrm{MIN}$. temperature now has risen to 248 degrees $F$. The Chromalox oil temperature set point is raised to 450 degrees $F$.

4 HOURS

Six gallons total is now drawn off the knock-out pot. Material temperature is $30 \mathrm{MIN}$. 256 degrees $\mathrm{F}$. The oil temperature is 450 degrees $\mathrm{F}$. As the solvent is drawn from the coal the viscosity is increasing slightly, the stirrer blades are still only drawing 11 amps on the $15 \mathrm{HP}$ motor at $20 \mathrm{rpm}$.

5 HOURS Eleven gallons total now drawn from the condenser. For this product to be $30 \mathrm{MIN}$. thoroughly dry it would be necessary to draw off between $15-16$ gallons. Material temperature now 272 degrees $F$. Oil temperature maintained at 450 degrees $F$. With this amount of solvent drawn from the batch the viscosity is increasing and at this point the material takes on a taffy-like consistency. The amperage draw is still within limit of the $15 \mathrm{HP}$ motor.

6 HOURS The coal consistency now is a very heavy large mass that has balled up in between the stirrer blades. The upper vacuum hood and gearbox area begins to shift inside the mix can and a loud banging noise is heard. The machine is then stopped. The atch is cooled to approximately 140 degrees $F$. The vacuum is then broken. The remainder of the solvent is drained from the Helex condenser and when the material is cool enough the vacuum hood is raised. Our material is now is solid although very friable mass of carbon which easily breaks up in the hand. The product resembles a very fine graphite powder. This is chiseled off the stirrer blades and placed in five-gallon buckets to be returned to the University of WV.

Phone: 631/234-0500 - Fax: 631/234-0691 - e-mail: mail@rossmixing.com 